Adv. Geom. 9 (2009), 471-515

DOI 10.1515/ ADVGEOM.2009.029

Advances in Geometry

(c) de Gruyter 2009

\title{
Sous-espaces équi-isoclins de l'espace euclidien
}

\author{
Boumediene Et-Taoui and Augustin Fruchard \\ (Communicated by K. Strambach)
}

\begin{abstract}
A systematic study of equi-isoclinic $n$-tuples of the Grassmann manifold $G(d, N)$ is initiated. After basic results in the general case, the article focuses on the case $d=2$. In particular the lists of all regular equi-isoclinic $n$-tuples of $G(2,2 n)$ and of all equi-isoclinic quadruples of $G(2,6)$ are established.
\end{abstract}

Key words. Grassmann manifold, equi-isoclinic, Seidel M-matrix, holonomy, packing, distance geometry.

2000 Mathematics Subject Classification. 51M20, 51M35, 51K99, 51F20

\section{Introduction}

La position mutuelle de deux sous-espaces vectoriels de dimension $d$ de l'espace euclidien $\mathbb{R}^{N}$ est entièrement déterminée par la connaissance de leurs angles critiques $\theta_{1}, \ldots, \theta_{d} \in\left[0, \frac{\pi}{2}\right]$. Ce sont les valeurs critiques de la fonction qui, à un vecteur de l'un des sous-espaces vectoriels, associe l'angle entre ce vecteur et son projeté orthogonal sur l'autre sous-espace. Notons que les cosinus de ces angles critiques sont les longueurs des demi-axes de l'ellipsoïde image de la sphère unité de l'un des sous-espaces par la projection orthogonale dans l'autre. Lorsque tous les angles critiques sont égaux, autrement dit lorsque l'ellipsoïde est une sphère, on dit que les deux sous-espaces sont isoclins de paramètre $c=\cos \theta_{1}=\cdots=\cos \theta_{d}$. La projection orthogonale de l'un dans l'autre est alors une similitude de rapport $c$. Un $n$-uplet $\left(\Gamma_{1}, \ldots, \Gamma_{n}\right)$ de la variété de Grassmann $G(d, N)$ est dit équi-isoclin de paramètre $c$ si chaque paire $\left(\Gamma_{i}, \Gamma_{j}\right)$ est isocline avec le même paramètre $c$.

L'objet de ce travail est l'étude des classes de congruence de $n$-uplets de sous-espaces de dimension $d$ équi-isoclins de l'espace euclidien $\mathbb{R}^{N}$. Puisque nous ne considérerons que des $n$-uplets équi-isoclins dans cet article, le mot "équi-isoclin" sera le plus souvent omis et systématiquement sous-entendu. Le fil conducteur de notre étude est d'apporter des éléments de réponse aux problèmes suivants. 
- Trouver un système complet d'invariants par congruence d'un $n$-uplet.

- Déterminer l'ordre de superposabilité de la variété de Grassmann $G(d, N)$, c'est-àdire le nombre minimal $k$ tel qu'un $n$-uplet de $G(d, N)$ est déterminé à congruence près par ses sous $k$-uplets (on n'examinera que le cas des $n$-uplets équi-isoclins).

- Etablir la liste exhaustive des $n$-uplets réguliers, c'est-à-dire ceux ayant un groupe de symétrie isomorphe au groupe symétrique $\mathcal{S}_{n}$.

- Problème de remplissage (en anglais, "packing") : trouver le plus grand nombre possible de sous-espaces inclus dans un espace de dimension $N$ donné. De manière plus précise, étant donnés $n, d, N \in \mathbb{N}^{*}$, établir la liste des $n$-uplets (équi-isoclins pour cet article) de $G(d, N)$.

Etant donnée l'ampleur des problèmes posés, nous ne pouvons donner que des réponses très partielles. Dans cet article, une attention particulière est portée au cas $d=2$, c'est-à-dire à l'étude des $n$-uplets de plans. Les cas $d=3$ et $d=4$ sont très riches et feront l'objet d'un article ultérieur.

Notre outil principal est l'utilisation des M-matrices associées à un tel $n$-uplet, une notion introduite par P.W.H. Lemmens et J.J. Seidel dans [12]. Ce sont des matrices de $n \times n$ blocs carrés d'ordre $d$ chacun, symétriques, dont les blocs sur la diagonale sont tous nuls et les autres sont des matrices orthogonales. En mémoire de J.J. Seidel, nous avons choisi d'appeler ces M-matrices matrices de Seidel.

Le deuxième outil est la notion d'holonomie : étant donnés un $n$-uplet $\left(\Gamma_{1}, \ldots, \Gamma_{n}\right)$ et un cycle $\gamma=\left(i_{1} \ldots i_{k}\right)$ de $\mathcal{S}_{n}$, l'holonomie le long de $\gamma$ est l'opération qui consiste à transporter parallèlement un repère de $\Gamma_{i_{1}}$ successivement dans $\Gamma_{i_{k}}, \Gamma_{i_{k-1}}, \ldots$ jusqu'à $\Gamma_{i_{1}}$. Nous montrons que ces holonomies sont des invariants par congruence du $n$-uplet.

Concernant notre premier problème, nous apportons une réponse dans deux cas : les triplets de sous-espaces de dimension $d$ arbitraire et les quadruplets de plans. Précisément, nous démontrons d'une part que la classe de congruence d'un triplet de $G(d, 3 d)$ est déterminée par son paramètre $c$ et par son holonomie le long d'un cycle de longueur 3 , et d'autre part que la classe de congruence d'un quadruplet de $G(2,8)$ est déterminée par son paramètre $c$ et par ses holonomies le long des cycles de longueurs 3 et 4 . Nous donnons aussi un exemple montrant que ce résultat pour les plans ne s'étend pas en dimension supérieure. Pour cette raison, nous introduisons la notion de pseudo-régularité.

Concernant notre deuxième problème, l'ordre de superposabilité a été introduit dans [1] et les résultats connus sont les suivants. Pour $n \geq 2$, l'ordre de superposabilité de l'espace euclidien, sphérique ou hyperbolique de dimension $n$ est égal à 2 [1]. Celui de l'espace projectif réel de dimension $n$ est égal à 3 , si l'on suppose que les distances mutuelles entre les points sont inférieures à $\frac{\pi}{2}[1]$. Avec les mêmes restrictions sur les distances mutuelles, l'ordre de superposabilité de l'espace projectif complexe de dimension $n$ est égal à 4 [3], et celui de l'espace projectif quaternionien de dimension $n$ est au moins 6 [4]. Dans cet article, nous montrons que l'ordre de superposabilité de $G(2, N)$ est au moins 5 , c.f. item 3 du théorème 5.6.

Nous répondons à notre troisième problème dans le cas $d=2$ : nous dressons la liste complète des $n$-uplets réguliers de $G(2,2 n)$.

Notre quatrième problème n'est abordé lui aussi que dans le cas $n=2$, et seulement dans $\mathbb{R}^{6}$. Même pour ce cas très restrictif, nos réponses sont très partielles : nous 
avons établi la liste des quadruplets de $G(2,6)$, mais nous n'avons pas examiné ceux qui s'étendent en des quintuplets de $G(2,6)$. Nous sommes donc encore très loin d'avoir exploré tous les $n$-uplets de $G(2,6)$ jusqu'à leur maximum : le 9-uplet correspondant au 9 -uplet de droites équi-angulaires dans $\mathbb{C}^{3}$, c.f. [8]. Enfin, mentionnons [5] pour le problème plus général de remplissage avec des $n$-uplets non nécessairement équi-isoclins, [17] pour l'étude des $n$-uplets de $d$-plans de l'espace euclidien de dimension $2 d$, isoclins deux à deux non nécessairement équi-isoclins et [11] pour des généralisations de certains résultats de P.W.H. Lemmens et J.J. Seidel aux cas de l'espace complexe et de l'espace quaternionien.

L'organisation de l'article est la suivante. Dans la partie 2, nous définissons une relation d'équivalence sur les matrices de Seidel et nous montrons que deux $n$-uplets sont congruents si, et seulement si, ils ont des matrices de Seidel associées équivalentes. Nous démontrons aussi que toute matrice de Seidel peut être associée à certains $n$-uplets. En relation avec notre quatrième problème sur le remplissage, nous définissons l'ordre de platitude d'un $n$-uplet et nous établissons le lien avec le spectre d'une matrice de Seidel associée au $n$-uplet. Certains résultats de cette partie 2 sont déjà connus ; dans ce cas cela est mentionné. Pour la complétude de l'article, nous avons joint toutes les preuves.

Dans cette partie 2 nous introduisons aussi notre notion d'holonomie et présentons quelques résultats s'y rattachant, puis nous montrons comment le produit tensoriel permet la construction de $n$-uplets de $G(d, n d)$ qui contiennent des $n$-uplets de droites ou de plans, avec le même paramètre.

Les parties 3 et 4 concernent l'étude des triplets et des quadruplets. Pour le triplet, nous montrons qu'il est toujours régulier et nous déterminons son ordre de platitude en fonction de son holonomie le long d'un cycle de longueur 3. Concernant le quadruplet, nous donnons une condition nécessaire et suffisante de pseudo-régularité, et distinguons trois types.

La partie 5 est consacrée aux deux premières questions dans le cas $d=2$ : nous démontrons que les holonomies forment un système complet d'invariants par congruence, puis nous montrons que les $n$-uplets de plans de type I ou II sont déterminés par leurs sous-quadruplets, mais pas les $n$-uplets d'un type mixte (le type d'un $n$-uplet est décrit dans la définition 4.3).

Dans la partie 6, nous dressons la liste complète des quadruplets réguliers de $G(2,8)$, puis la liste complète des $n$-uplets réguliers de $G(2,2 n)$.

Dans la dernière partie 7 , nous déterminons tous les quadruplets de $G(2,6)$, avec leur ordre de platitude. Dans le cas des quadruplets de type mixte, la complexité des calculs a nécessité le recours à l'ordinateur. Nous précisons cependant que celui-ci n'a servi que pour des calculs purement formels (calcul de déterminants et factorisation de polynômes) et que, hormis deux calculs particulièrement longs, tous ces calculs ont été effectués aussi à la main. Nous espérons donc que le lecteur pourra considérer nos preuves comme complètes.

Remerciements. Nous remercions chaleureusement Théodor HANGAN et Reinhard SCHÄFKE pour de nombreuses et fructueuses discussions à propos de ce travail. 


\section{Généralités}

2.1 Conventions et notations. Dans tout l'article, $n, d$ et $N$ sont des nombres entiers. $G(d, N)$ désigne la variété de Grassmann des sous-espaces de dimension $d$ dans $\mathbb{R}^{N}$.

Rappelons que tous les $n$-uplets de l'article sont équi-isoclins, et que cette propriété sera systématiquement sous-entendue. On considère un $n$-uplet $\left(\Gamma_{1}, \ldots, \Gamma_{n}\right)$ ordonné de $G(d, N)$ de paramètre $c \in] 0,1]$; autrement dit, $\Gamma_{1}, \ldots, \Gamma_{n}$ sont des sous-espaces vectoriels de dimension $d$ de $\mathbb{R}^{N}$, et les projections orthogonales de l'un dans l'autre sont toutes des similitudes de même rapport $c$. Nous serons parfois amenés à considérer la valeur $c=1$, correspondant au cas trivial où tous les éléments du $n$-uplet sont confondus. En revanche la valeur $c=0$, qui correspondrait à des sous-espaces $\Gamma_{j}$ deux à deux orthogonaux, est exclue pour des raisons qui apparaîtront clairement dans la suite.

Vect $(E)$ désigne le sous-espace vectoriel engendré par une partie $E$ de $\mathbb{R}^{N}$.

${ }^{t} A$ désigne la transposée d'une matrice $A$.

$\operatorname{Diag}\left(B_{1}, \ldots, B_{n}\right)$ désigne une matrice diagonale par blocs, où les blocs $B_{1}, \ldots, B_{n}$ sont des matrices carrées, pas nécessairement de même ordre.

Etant donnés $p$ vecteurs $\vec{v}_{1}, \ldots, \vec{v}_{p}$ de $\mathbb{R}^{N}$, la matrice de Gram de $\vec{v}_{1}, \ldots, \vec{v}_{p}$, notée $\operatorname{Gram}\left(\vec{v}_{1}, \ldots, \vec{v}_{p}\right)$, est la matrice des produits scalaires :

$$
\operatorname{Gram}\left(\vec{v}_{1}, \ldots, \vec{v}_{p}\right)=\left(\begin{array}{ccc}
\vec{v}_{1} \cdot \vec{v}_{1} & \ldots & \vec{v}_{1} \cdot \vec{v}_{p} \\
\vdots & \ddots & \vdots \\
\vec{v}_{p} \cdot \vec{v}_{1} & \ldots & \vec{v}_{p} \cdot \vec{v}_{p}
\end{array}\right)
$$

Rappelons que, si $V$ est la matrice $N \times p$ constituée des coordonnées des vecteurs $\vec{v}_{1}, \ldots$, $\vec{v}_{p}$ dans une base orthonormée de $\mathbb{R}^{N}$, alors $\operatorname{Gram}\left(\vec{v}_{i}\right)={ }^{t} V V$, si bien que le rang de $\operatorname{Gram}\left(\vec{v}_{i}\right)$ est égal au rang de $V$, donc à la dimension de $\operatorname{Vect}\left(\left\{\vec{v}_{1}, \ldots, \vec{v}_{p}\right\}\right)$, et que les valeurs propres de $\operatorname{Gram}\left(\vec{v}_{i}\right)$ sont réelles, positives ou nulles.

$\mathcal{S}_{n}$ désigne le groupe des permutations de $\{1, \ldots, n\}$. Les permutations seront toujours notées par leur décomposition cyclique.

$\mathcal{O}_{\nu}$ désigne le groupe des matrices orthogonales $\nu \times \nu, \mathcal{O}_{\nu}^{+}$est le sous-groupe de celles de déterminant 1 et $\mathcal{O}_{\nu}^{-}=\mathcal{O}_{\nu} \backslash \mathcal{O}_{\nu}^{+}$.

$I_{\nu}$ est la matrice identité d'ordre $\nu$, souvent notée simplement $I$ lorsque le contexte permet de connaître son ordre sans ambiguité.

Rappelons que, si $A, B \in \mathcal{O}_{\nu}$ sont semblables (i.e. $\exists P \in \mathrm{GL}_{n}(\mathbb{R}), A P=P B$ ) alors on peut les conjuguer par une matrice orthogonale (i.e. $P^{t} P=I$ ). Nous dirons que $A$ et $B$ sont conjuguées et nous écrirons $A \sim B$.

Pour $A \in \mathcal{O}_{\nu}, \operatorname{cl}(A)$ désigne la classe de conjugaison de $A: \operatorname{cl}(A)=\left\{B \in \mathcal{O}_{\nu}\right.$; $B \sim A\}$.

Il est connu que toute matrice de $\mathcal{M}_{\nu}(\mathbb{R})$ est semblable à sa transposée. Il s'ensuit que si $A \in \mathcal{O}_{\nu}$ alors $\operatorname{cl}(A)$ contient ${ }^{t} A$.

Nous utiliserons dans la partie 7 le résultant et le discriminant de polynômes. Rappelons que le résultant de deux polynômes $P(x)=a_{0}+\cdots+a_{m} x^{m}$ et $Q(x)=$ $b_{0}+\cdots+b_{n} x^{n}, a_{m}$ et $b_{n}$ non nuls, est le déterminant d'ordre $m+n$ obtenu en écrivant les coefficients de $P$ dans $n$ colonnes décalées, puis ceux de $Q$ dans $m$ colonnes 
décalées :

$$
\operatorname{Res}(P, Q)=\left|\begin{array}{ccccccc}
a_{m} & & 0 & b_{n} & 0 & \cdots & 0 \\
a_{m-1} & \ddots & & \vdots & b_{n} & \ddots & \vdots \\
\vdots & & a_{m} & b_{0} & \vdots & \ddots & 0 \\
a_{0} & & \vdots & 0 & b_{0} & & b_{n} \\
& \ddots & a_{1} & \vdots & \ddots & \ddots & \vdots \\
0 & & a_{0} & 0 & \cdots & 0 & b_{0}
\end{array}\right| .
$$

Il est connu que, dans un corps où les deux polynômes sont scindés, i.e. lorsque

$$
P(x)=a_{m}\left(x-\xi_{1}\right) \ldots\left(x-\xi_{m}\right) \text { et } Q(x)=b_{n}\left(x-\eta_{1}\right) \ldots\left(x-\eta_{n}\right)
$$

(éventuellement avec des racines multiples), alors ce résultant est

$$
\operatorname{Res}(P, Q)=a_{m}^{n} b_{n}^{m} \prod_{\substack{1 \leq i \leq m \\ 1 \leq j \leq n}}\left(\xi_{i}-\eta_{j}\right) .
$$

Dans cet article, le discriminant d'un polynôme $P(x)=a_{0}+\cdots+a_{m} x^{m}$ est donné par

$$
\Delta(P)=\frac{1}{a_{m}}(-1)^{(m-1) m / 2} \operatorname{Res}\left(P, P^{\prime}\right) .
$$

Nous avons adopté cette définition de façon à coller avec la définition en usage pour les polynômes de degré deux :

$$
\Delta\left(a x^{2}+b x+c\right)=-\frac{1}{a}\left|\begin{array}{ccc}
a & 2 a & 0 \\
b & b & 2 a \\
c & 0 & b
\end{array}\right|=b^{2}-4 a c .
$$

Avec ce choix, un polynôme scindé sur $\mathbb{R}$ a un discriminant positif ou nul. En effet, si $P$ est unitaire, de la forme $P(x)=\left(x-\xi_{1}\right) \ldots\left(x-\xi_{m}\right)$ avec $\xi_{1} \leq \xi_{2} \leq \cdots \leq$ $\xi_{m}$, alors le théorème de Rolle entraîne que $P^{\prime}$ a une racine $\eta_{i}$ dans chaque segment $\left[\xi_{i}, \xi_{i+1}\right]$, donc est scindé sur $\mathbb{R}$, et de plus le signe de $\prod_{\substack{i=1, \ldots, m \\ j=1, \ldots, m-1}}\left(\xi_{i}-\eta_{j}\right)$ est égal à $(-1)^{(m-1)+(m-2)+\cdots+2+1}=(-1)^{(m-1) m / 2}$. Dans le cas général, on utilise $\Delta(c P)=$ $c^{2 m-2} \Delta(P)$.

Par ailleurs, si $P$ et $Q$ sont deux polynômes unitaires de degré $m>1$ et si $k$ est un nombre réel non nul tel que pour tout $x \in \mathbb{R}$ on ait $Q(k x)=k^{m} P(x)$, alors avec les notations précédentes, les zéros de $Q$ et de $Q^{\prime}$ sont respectivement $k \xi_{i}$ et $k \eta_{j}$. Il s'ensuit qu'on a

$$
\Delta(Q)=k^{(m-1) m} \Delta(P) .
$$

\subsection{Ordre de platitude et matrice de Seidel.}

Définition 2.1. L'ordre de platitude d'un $n$-uplet $\left(\Gamma_{j}\right)_{j=1, \ldots, n}$ de $\mathbb{R}^{N}$ est égal à $n d-$ $\operatorname{dim} \operatorname{Vect}\left(\Gamma_{1} \cup \cdots \cup \Gamma_{n}\right)$. Dans le cas où $N=n d$, c'est donc la codimension de $\operatorname{Vect}\left(\Gamma_{1} \cup\right.$ $\left.\cdots \cup \Gamma_{n}\right)$. 
Définition 2.2. On appelle matrice de Seidel une matrice carrée d'ordre $n d$, où $n \geq 2$ et $d \geq 1$ sont deux entiers, de la forme

$$
M=\left(\begin{array}{cccc}
0 & A_{12} & \ldots & A_{1 n} \\
{ }^{t} A_{12} & 0 & \ddots & \vdots \\
\vdots & \ddots & \ddots & A_{n-1 n} \\
{ }^{t} A_{1 n} & \ldots & { }^{t} A_{n-1 n} & 0
\end{array}\right),
$$

où $A_{i j} \in \mathcal{O}_{d}$ pour $1 \leq i<j \leq n$ et 0 désigne la matrice nulle d'ordre $d$.

Cette notion a été introduite par P.W.H. Lemmens et J.J. Seidel dans [12]. Ce même article [12] contient aussi les items 1, 3 et 4 ci-dessous. En revanche, l'item 2 est nouveau à notre connaissance. Une preuve analogue peut être faite pour des $n$-uplets qui ne sont pas nécessairement équi-isoclins, ce qui répondrait positivement à une question de [9] (c.f. commentaire (3) p.138). Cependant nous avons choisi dans cet article de ne pas sortir du cadre équi-isoclin.

Théorème 2.3. 1. Soit $\left(\Gamma_{1}, \ldots, \Gamma_{n}\right)$ un n-uplet de paramètre c. Pour chaque $j \in\{1, \ldots$, $n\}$, soit $\left(u_{j}^{1}, \ldots, u_{j}^{d}\right)$ une base orthonormée de $\Gamma_{j}$. Alors la matrice de Gram de la famille $\left(u_{1}^{1}, \ldots, u_{1}^{d}, u_{2}^{1}, \ldots, u_{2}^{d}, \ldots, u_{n}^{1}, \ldots, u_{n}^{d}\right)$ est de la forme

$$
\operatorname{Gram}\left(u_{j}^{\nu}\right)=I+c M
$$

où $M$ est une matrice de Seidel et I désigne la matrice identité d'ordre nd. On dira dans ce cas que la matrice $M$ est associée $\grave{a}\left(\Gamma_{j}\right)$.

2. La plus petite valeur propre de $M$, notée $\mu_{0}$, satisfait

$$
1-n \leq \mu_{0} \leq-1 .
$$

Si $\mu_{0}=-1$, alors les matrices orthogonales $A_{i j}$ constituant $M$ satisfont pour tous $i, j, k$ distincts :

$$
A_{i j} A_{j k} A_{k i}=I .
$$

3. On a nécessairement $c \leq-\frac{1}{\mu_{0}}$. Si $c<-\frac{1}{\mu_{0}}$ alors l'ordre de platitude de $\left(\Gamma_{j}\right)$ est nul. Si $c=-\frac{1}{\mu_{0}}$ alors cet ordre de platitude est égal à la multiplicité de $\mu_{0}$ comme valeur propre de $M$.

4. Réciproquement, étant donnée une matrice de Seidel $M$, de plus petite valeur propre $\mu_{0}$, et $\left.\left.c \in\right] 0,-\frac{1}{\mu_{0}}\right]$, il existe un $n$-uplet $\left(\Gamma_{j}\right)$ de $G(d, n d)$ de paramètre $c$ et de matrice associée $M$.

Preuve. 1. Notons $\pi_{i j}$ la projection orthogonale de $\Gamma_{j}$ dans $\Gamma_{i}$ (attention à l'ordre); c'est une similitude de rapport $c$. Soit $A_{i j}$ la matrice de l'isométrie $\frac{1}{c} \pi_{i j}$ dans les bases respectives $\left(u_{i}^{1}, \ldots, u_{i}^{d}\right)$ et $\left(u_{j}^{1}, \ldots, u_{j}^{d}\right)$. La matrice de terme général $u_{i}^{p} \cdot u_{j}^{q}, 1 \leq p, q \leq d$ est précisément la matrice de $\pi_{i j}$, donc égale à $c A_{i j}$ et la matrice de terme général $u_{i}^{p} \cdot u_{i}^{q}$, 
$1 \leq p, q \leq d$ est la matrice identité puisque $\left(u_{j}^{1}, \ldots, u_{j}^{d}\right)$ est une base orthonormée de $\Gamma_{j}$. Par conséquent, $\operatorname{Gram}\left(u_{j}^{\nu}\right)=I+c M$ avec $M$ donnée par (2).

2. $M$ étant une matrice symétrique non nulle et de trace nulle, on a $\mu_{0}<0$. Soit $\vec{V}=$ $\left(\begin{array}{c}\vec{v}_{1} \\ \vdots \\ \vec{v}_{n}\end{array}\right) \in \mathbb{R}^{n d}$ un vecteur propre de $M$ associé à la valeur propre $\mu_{0}$. Soit $i$ tel que $\left\|\vec{v}_{i}\right\|$ soit maximale parmi $\left\{\left\|\vec{v}_{1}\right\|, \ldots,\left\|\vec{v}_{n}\right\|\right\}$ (donc non nulle). On a $A_{i 1} \vec{v}_{1}+\cdots \widehat{i} \cdots+A_{i n} \vec{v}_{n}=$ $\mu_{0} \vec{v}_{i}$, où $\hat{i}$ signifie que le terme d'indice $i$ a été sauté. On en déduit

$$
\begin{aligned}
\left|\mu_{0}\right|\left\|\vec{v}_{i}\right\| & =\left\|A_{i 1} \vec{v}_{1}+\cdots \widehat{i} \cdots+A_{i n} \vec{v}_{n}\right\| \leq\left\|A_{i 1} \vec{v}_{1}\right\|+\cdots \widehat{i} \cdots+\left\|A_{i n} \vec{v}_{n}\right\| \\
& =\left\|\vec{v}_{1}\right\|+\cdots \hat{i} \cdots+\left\|\vec{v}_{n}\right\| \leq(n-1)\left\|\vec{v}_{i}\right\|,
\end{aligned}
$$

d'où $\left|\mu_{0}\right| \leq n-1$, d'où $\mu_{0} \geq 1-n$. L'inégalité $\mu_{0} \leq-1$ et le cas $\mu_{0}=-1$ seront démontrés plus bas.

3. Notons $G$ la matrice de Gram des vecteurs $u_{j}^{i}$. Puisque $G=I+c M$, les valeurs propres de $G$ sont exactement les valeurs $1+c \lambda_{k}, 1 \leq k \leq n d$ où $\lambda_{1}, \ldots, \lambda_{n d}$ sont les valeurs propres de $M$. En particulier la plus petite valeur propre de $G$ est égale à $1+c \mu_{0}$, avec la même multiplicité $m\left(\mu_{0}\right)$ que $\mu_{0}$ pour $M$. Puisque $G$ est une matrice de Gram, toutes ses valeurs propres, en particulier $1+c \mu_{0}$, sont positives ou nulles; or $\mu_{0}<0$, donc $c \leq-\frac{1}{\mu_{0}}$. Par ailleurs, il est connu que la dimension de $\operatorname{Vect}\left(\Gamma_{1} \cup \cdots \cup \Gamma_{n}\right)=\operatorname{Vect}\left(u_{j}^{i}\right)$ est égale au rang de $G$.

Si $c<-\frac{1}{\mu_{0}}$ alors la plus petite valeur propre de $G$, à savoir $1+c \mu_{0}$, est strictement positive, donc $G$ est de rang maximal $n d$.

Si $c=-\frac{1}{\mu_{0}}$, alors 0 est valeur propre de $G$, de multiplicité égale à $m\left(\mu_{0}\right)$, donc le rang de $G$ est égal à $n d-m\left(\mu_{0}\right)$, donc l'ordre de platitude du $n$-uplet est égal à $m\left(\mu_{0}\right)$.

4. Soit $G$ la matrice symétrique $G=I+c M$. Puisque $\left.c \in] 0,-\frac{1}{\mu_{0}}\right]$, on a $1+c \lambda \geq 0$ pour toute valeur propre $\lambda$ de $M$. Ainsi $G$ est aussi positive; il existe donc $n d$ vecteurs $u_{1}^{1}, \ldots, u_{1}^{d}, \ldots, u_{n}^{1}, \ldots, u_{n}^{d} \in \mathbb{R}^{N}$ dont $G$ est la matrice de Gram. Soit alors, pour chaque $j \in\{1, \ldots, n\}, \Gamma_{j}$ le sous-espace engendré par $\left\{u_{j}^{1}, \ldots, u_{j}^{d}\right\}$. On obtient ainsi un $n$-uplet $\left(\Gamma_{j}\right)_{1 \leq j \leq n}$ de paramètre $c$ et, dans chaque $\Gamma_{j}$, une base orthonormée $\left(u_{j}^{1}, \ldots, u_{j}^{d}\right)$ tels que la matrice de Gram de la famille $\left(u_{j}^{i}\right)$ soit égale à $G$.

Fin de la preuve du point 2 . Supposons $\mu_{0} \geq-1$. D'après le point précédent, cela entraîne que pour tout $c \in] 0,1]$ il existe un $n$-uplet $\left(\Gamma_{1}, \ldots, \Gamma_{n}\right)$ de paramètre $c$ et de matrice de Seidel associée $M$. Pour la valeur $c=1$, tous les sous-espaces $\Gamma_{j}$ sont confondus, donc les projections $\pi_{i j}$ sont en fait l'identité de $\Gamma_{1}$ dans lui-même, si bien que les matrices $A_{i j}$ sont des matrices de changements de base. Précisément, si on fixe $\left(e^{1}, \ldots, e^{d}\right)$ une base de $\Gamma_{1}$ et si on note $U_{j}$ la matrice $d \times d$ dont les colonnes sont les coordonnées des vecteurs $u_{j}^{\nu}$ dans cette base, alors $A_{i j}={ }^{t} U_{i} U_{j}$ pour tout $i \neq j$. Puisque $\left(u_{j}^{1}, \ldots, u_{j}^{d}\right)$ est une base orthonormée de $\Gamma_{1}$, la matrice $U_{j}$ est dans $\mathcal{O}_{d}$, donc $U_{j}{ }^{t} U_{j}=I$. L'identité (5) s'en déduit.

A présent, soit $P:=\operatorname{Diag}\left(I, A_{12}, \ldots, A_{1 n}\right)$. Un simple calcul montre que $P M P^{-1}$ est la matrice de Seidel dont les blocs non nuls sont tous égaux à l'identité. Comme nous 
le verrons dans la section 2.5, cette matrice a pour valeurs propres $\lambda_{1}=-1$ avec la multiplicité $(n-1) d$ et $\lambda_{2}=n-1$ avec la multiplicité $d$. On obtient ainsi $\mu_{0}=-1$.

Ainsi, on a montré d'une part que, si $\mu_{0} \geq-1$, alors $\mu_{0}=-1$, ce qui montre que $\mu_{0} \leq-1$, et d'autre part que dans le cas où $\mu_{0}=-1$, les identités (5) sont satisfaites.

\subsection{Congruence de $n$-uplets et matrices de Seidel équivalentes.}

Définition 2.4. On dit que deux $n$-uplets $\left(\Gamma_{j}\right)_{j=1, \ldots, n}$ et $\left(\widetilde{\Gamma}_{j}\right)_{j=1, \ldots, n}$ de $G(d, N)$ sont congruents s'il existe une isométrie $\Phi$ de $\mathbb{R}^{N}$ telle que pour tout $j \in\{1, \ldots, n\}$ on ait $\widetilde{\Gamma}_{j}=\Phi\left(\Gamma_{j}\right)$. Dans ce cas on note $\left(\Gamma_{j}\right) \approx\left(\widetilde{\Gamma}_{j}\right)$.

Soit $\left(\Gamma_{j}\right)$ un $n$-uplet de matrice de Seidel associée $M$. Il est opportun de considérer $M$ comme une matrice par blocs, i.e. une matrice $n \times n$ à coefficients dans $\mathcal{M}_{d}(\mathbb{R})$. Ainsi nous appellerons la $i$-ième ligne de $M$ la ligne bloc correspondant à $\Gamma_{i}$.

A un changement de base orthonormée dans $\Gamma_{j}$, de matrice de passage $P \in \mathcal{O}_{d}$, correspond la multiplication de la $j$-ième ligne de $M$ par ${ }^{t} P$ et de la $j$-ième colonne par $P$. Plus généralement, soit $\left(v_{1}^{1}, \ldots, v_{1}^{d}\right), \ldots,\left(v_{n}^{1}, \ldots, v_{n}^{d}\right)$ d'autres bases orthonormées de $\Gamma_{1}, \ldots, \Gamma_{n}$ respectivement et soit $\widetilde{M}$ la matrice de Seidel associée à $\left(\Gamma_{j}\right)$ dans ces nouvelles bases, dont les blocs non nuls sont notés $\widetilde{A}_{i j}$. Pour chaque $j$, soit $P_{j} \in \mathcal{O}_{d}$ la matrice de passage de $\left(u_{j}^{1}, \ldots, u_{j}^{d}\right)$ à $\left(v_{j}^{1}, \ldots, v_{j}^{d}\right)$. Alors pour tout $i, j \in\{1, \ldots, n\}$, $i \neq j$ on a

$$
{ }^{t} P_{i} A_{i j} P_{j}=\widetilde{A}_{i j} .
$$

Réciproquement, si un $n$-uplet $\left(\Gamma_{1}, \ldots, \Gamma_{n}\right)$ a une matrice de Seidel associée constituée de blocs non nuls $A_{i j}$ et si $\widetilde{A}_{i j}$ sont tels qu'il existe $P_{1}, \ldots, P_{n}$ satisfaisant (7), alors la matrice de Seidel constituée des $\widetilde{A}_{i j}$ est aussi associée à $\left(\Gamma_{1}, \ldots, \Gamma_{n}\right)$. Ceci motive la définition suivante.

Définition 2.5. Deux matrices de Seidel $M$ et $\widetilde{M}$ sont dites équivalentes s'il existe $P_{1}$, $\ldots, P_{n} \in \mathcal{O}_{d}$ tels que pour tout $i, j \in\{1, \ldots, n\}, i \neq j$ on ait (7).

Ainsi deux matrices de Seidel sont équivalentes si et seulement si elles sont associées à un même $n$-uplet; il s'agit donc bien d'une relation d'équivalence.

Théorème 2.6. Soit $\left(\Gamma_{j}\right)$ et $\left(\widetilde{\Gamma}_{j}\right)$ deux n-uplets de même paramètre $c \neq 0$ et de matrices associées respectives $M$ et $\widetilde{M}$. On a $\left(\Gamma_{j}\right) \approx\left(\widetilde{\Gamma}_{j}\right)$ si et seulement si les matrices $M$ et $\widetilde{M}$ sont équivalentes.

Preuve. $\mathrm{Si}\left(\Gamma_{j}\right) \approx\left(\widetilde{\Gamma}_{j}\right)$, alors il existe une isométrie $\Phi$ de $\mathbb{R}^{N}$ telle que pour tout $j \in$ $\{1, \ldots, n\}$ on ait $\widetilde{\Gamma}_{j}=\Phi\left(\Gamma_{j}\right)$. Soient $\left(u_{1}^{1}, \ldots, u_{1}^{d}\right), \ldots,\left(u_{n}^{1}, \ldots, u_{n}^{d}\right)$ des bases orthonormées respectives de $\Gamma_{1}, \ldots, \Gamma_{n}$. Posons $v_{j}^{i}=\Phi\left(u_{j}^{i}\right)$; ainsi $\left(v_{j}^{1}, \ldots, v_{j}^{d}\right)$ est une base orthonormée de $\widetilde{\Gamma}_{j}$. Puisque $\Phi$ conserve le produit scalaire, on a $\operatorname{Gram}\left(u_{j}^{i}\right)=\operatorname{Gram}\left(v_{j}^{i}\right)$, donc $M$ est aussi une matrice de Seidel associée à $\left(\widetilde{\Gamma}_{j}\right)$. D'après ce qui suit la définition $2.5, M$ et $\widetilde{M}$ sont donc équivalentes. 
Réciproquement, si $M$ et $\widetilde{M}$ sont équivalentes, soient $\left(u_{1}^{1}, \ldots, u_{1}^{d}\right), \ldots,\left(u_{n}^{1}, \ldots, u_{n}^{d}\right)$ des bases orthonormées respectives de $\Gamma_{1}, \ldots, \Gamma_{n}$ telles que $\operatorname{Gram}\left(u_{j}^{i}\right)=I+c M$. Posons

$$
\left(\begin{array}{c}
v_{j}^{1} \\
\vdots \\
v_{j}^{d}
\end{array}\right)={ }^{t} P_{j}\left(\begin{array}{c}
u_{j}^{1} \\
\vdots \\
u_{j}^{d}
\end{array}\right) .
$$

Alors $\left(v_{1}^{1}, \ldots, v_{1}^{d}\right), \ldots,\left(v_{n}^{1}, \ldots, v_{n}^{d}\right)$ forment $n$ bases de $\Gamma_{1}, \ldots, \Gamma_{n} \operatorname{avec} \operatorname{Gram}\left(v_{j}^{i}\right)=$ $I+c \widetilde{M}$. Ainsi $\widetilde{M}$ est une matrice associée à $\left(\Gamma_{j}\right)$. Soient maintenant $\left(\widetilde{u}_{1}^{1}, \ldots, \widetilde{u}_{1}^{d}, \ldots, \widetilde{u}_{n}^{1}\right.$, $\left.\ldots, u_{n}^{d}\right)$ des bases orthonormées respectives de $\widetilde{\Gamma}_{1}, \ldots, \widetilde{\Gamma}_{n}$ telles que $\operatorname{Gram}\left(\widetilde{u}_{j}^{i}\right)=I+$ $c \widetilde{M}$. Les deux familles de $\mathbb{R}^{N}\left(v_{1}^{1}, \ldots, v_{1}^{d}, \ldots, v_{n}^{1}, \ldots, v_{n}^{d}\right)$ et $\left(\widetilde{u}_{1}^{1}, \ldots, \widetilde{u}_{1}^{d}, \ldots, \widetilde{u}_{n}^{1}, \ldots\right.$, $\widetilde{u}_{n}^{d}$ ) ont donc les mêmes matrices de Gram. Il est connu qu'il existe alors une isométrie $\Phi$ de $\mathbb{R}^{N}$ telle que $\widetilde{u}_{j}^{i}=\Phi\left(v_{j}^{i}\right)$. Ainsi $\Phi$ envoie $\Gamma_{j}$ sur $\widetilde{\Gamma}_{j}$, donc $\left(\Gamma_{j}\right) \approx\left(\widetilde{\Gamma}_{j}\right)$.

Puisque $c$ est supposé non nul, le choix d'une base dans le premier sous-espace $\Gamma_{1}$ permet de fixer des bases dans les autres en projetant cette base, autrement dit en choisissant $u_{j}^{\nu}:=\frac{1}{c} \pi_{j 1}\left(u_{1}^{\nu}\right)$ pour $j=2, \ldots, n, \nu=1, \ldots, d$. Ceci revient à imposer à la matrice de Seidel associée à $\left(\Gamma_{j}\right)_{j=1, \ldots, n}$ d'être composée de matrices identités d'ordre $d$ sur la première ligne :

$$
M=\left(\begin{array}{ccccc}
0 & I & I & \ldots & I \\
I & 0 & A_{23} & \ldots & A_{2 n} \\
I & { }^{t} A_{23} & \ddots & \ddots & \vdots \\
\vdots & \vdots & \ddots & \ddots & A_{n-1 n} \\
I & { }^{t} A_{2 n} & \ldots & { }^{t} A_{n-1 n} & 0
\end{array}\right)
$$

Pour simplifier, on écrira une telle matrice $M\left(A_{23}, \ldots, A_{2 n}, A_{34}, \ldots, A_{n-1 n}\right)$ et on l'appellera forme réduite du $n$-uplet. Notons au passage que, si la plus petite valeur propre d'une forme réduite $M$ vaut -1 , alors l'identité (5) devient $A_{i j}=I$ pour tout $2 \leq i<$ $j \leq n$. Ainsi, $M(I, \ldots, I)$ est la seule forme réduite possible satisfaisant $\mu_{0}=-1$.

Cette forme réduite permet de simplifier le critère de congruence présenté dans le théorème 2.6.

Corollaire 2.7. Soit $\left(\Gamma_{j}\right)$ et $\left(\widetilde{\Gamma}_{j}\right)$ deux n-uplets de même paramètre $\left.\left.c \in\right] 0,1\right]$ et de formes réduites $M\left(A_{i j}\right)_{2 \leq i<j \leq n}$ et $M\left(\widetilde{A}_{i j}\right)_{2 \leq i<j \leq n}$ respectivement.

On a $\left(\Gamma_{j}\right) \approx\left(\widetilde{\Gamma}_{j}\right)$ si et seulement si les matrices $A_{i j}$ et $\widetilde{A}_{i j}$ sont simultanément conjuguées, autrement dit s'il existe une matrice $P \in \mathcal{O}_{d}$ telle que pour tout $i, j$ satisfaisant $2 \leq i<j \leq n$, on a $A_{i j} P=P \widetilde{A}_{i j}$.

Preuve. Supposons que $\left(\Gamma_{j}\right) \approx\left(\widetilde{\Gamma}_{j}\right)$. D'après le théorème 2.6, il existe $P_{1}, \ldots, P_{n} \in \mathcal{O}_{d}$ tels que pour tout $i, j \in\{1, \ldots, n\}, i \neq j$ on a ${ }^{t} P_{i} A_{i j} P_{j}=\widetilde{A}_{i j}$. En particulier pour $i=1$, puisque $A_{1 j}=\widetilde{A}_{1 j}=I$, on obtient $P_{j}=P_{1}$ pour tout $j \in\{2, \ldots, n\}$. La réciproque est immédiate par application du théorème 2.6 en posant $P_{1}=\cdots=P_{n}=P$. 
2.4 Holonomie et régularité. Etant donné un $n$-uplet $\left(\Gamma_{j}\right)_{j=1, \ldots, n}$ de paramètre $c$ et un cycle $\gamma=\left(i_{1} \ldots i_{k}\right)$ de $\mathcal{S}_{n}$, considérons l'application de $\Gamma_{i_{1}}$ dans lui-même qui consiste à projeter successivement de $\Gamma_{i_{1}}$ dans $\Gamma_{i_{k}}$, puis dans $\Gamma_{i_{k-1}}$, etc., enfin dans $\Gamma_{i_{1}}$. Puisque chacune de ces projections est une similitude de rapport $c$ en restriction aux sous-espaces $\Gamma_{j}$ adéquats, en normalisant par le facteur $c^{-k}$, on obtient une isométrie de $\Gamma_{i_{1}}$ dans luimême :

$$
c^{-k} \pi_{i_{1} i_{2}} \circ \cdots \circ \pi_{i_{k-1} i_{k}} \circ \pi_{i_{k} i_{1}}: \Gamma_{i_{1}} \rightarrow \Gamma_{i_{1}} .
$$

Cette application dépend bien entendu de l'écriture choisie pour le cycle, mais sa classe de conjugaison n'en dépend pas. En effet, les applications $\pi_{i_{1} i_{2}} \circ \cdots \circ \pi_{i_{k-1} i_{k}} \circ \pi_{i_{k} i_{1}}$ et $\pi_{i_{l} i_{l+1}} \circ \cdots \circ \pi_{i_{k} i_{1}} \circ \pi_{i_{1} i_{2}} \circ \cdots \circ \pi_{i_{l-1} i_{l}}$ sont conjuguées ( $\operatorname{par} \pi_{i_{1} i_{2}} \circ \cdots \circ \pi_{i_{l-1} i_{l}}$ ). La définition suivante est donc cohérente.

Définition 2.8. Etant donné un $n$-uplet $\left(\Gamma_{j}\right)_{j=1, \ldots, n}$ de paramètre $c$ et $\gamma=\left(i_{1} \ldots i_{k}\right)$ un cycle de $\mathcal{S}_{n}$, l'holonomie le long de $\gamma$ est donnée par

$$
h(\gamma):=\operatorname{cl}\left(c^{-k} \pi_{i_{1} i_{2}} \circ \cdots \circ \pi_{i_{k-1} i_{k}} \circ \pi_{i_{k} i_{1}}\right) .
$$

Matriciellement, si $M=\left(\begin{array}{ccc}0 & & A_{i j} \\ & \ddots & \\ A_{i j} & & 0\end{array}\right)$ est une matrice de Seidel associée au $n$ uplet, alors $h(\gamma)$ est la classe de conjugaison du produit $A_{i_{1} i_{2}} \ldots A_{i_{k-1} i_{k}} A_{i_{k} i_{1}}$. L'holonomie le long des transpositions est triviale, puisque $A_{i j} A_{j i}=I_{d}$.

Ces holonomies correspondent au transport parallèle d'un repère de $\Gamma_{i_{1}}$ le long de la géodésique joignant $\Gamma_{i_{1}}$ à $\Gamma_{i_{k}}$, puis le long de la géodésique joignant $\Gamma_{i_{k}}$ à $\Gamma_{i_{k-1}}$ et ainsi de suite jusqu'à le ramener dans $\Gamma_{i_{1}}$. Il est possible d'effectuer un tel transport parallèle le long de tout lacet sur le polyèdre de $G(d, N)$ constitué des sommets $\Gamma_{j}$ et des arêtes géodésiques joignant deux sommets. Cependant dans cet article nous avons volontairement restreint nos holonomies à des lacets correspondant à des cycles de $\mathcal{S}_{n}$, que l'on pourrait appeler des holonomies primaires.

Etant donné un $n$-uplet, l'ensemble constitué des holonomies le long de tous les cycles de longueur comprise entre 3 et $n$ est un invariant par congruence du $n$-uplet. Une question naturelle est de savoir si ces invariants suffisent ou non à déterminer la classe de congruence du $n$-uplet. Nous traiterons cette question en détails pour les triplets et les quadruplets.

Une autre question, très liée, est de préciser le lien entre les holonomies et le groupe de symétrie d'un $n$-uplet.

Définition 2.9. Un $n$-uplet $\left(\Gamma_{j}\right)_{j=1, \ldots, n}$ est dit régulier si son groupe de symétrie est maximal, isomorphe à $\mathcal{S}_{n}$, autrement dit si $\left(\Gamma_{j}\right) \approx\left(\Gamma_{\sigma(j)}\right)$ pour toute permutation $\sigma \in$ $\mathcal{S}_{n}$.

Bien entendu, un $n$-uplet régulier doit nécessairement avoir les mêmes holonomies que celui obtenu après permutation des sommets, mais en général la condition n'est pas suffisante. Ceci motive la définition suivante. 
Définition 2.10. Un $n$-uplet $\left(\Gamma_{j}\right)_{j=1, \ldots, n}$ est dit pseudo-régulier si, pour tout $\sigma \in \mathcal{S}_{n}$, les $n$-uplets $\left(\Gamma_{j}\right)_{j=1, \ldots, n}$ et $\left(\Gamma_{\sigma(j)}\right)_{j=1, \ldots, n}$ ont les mêmes holonomies.

Proposition 2.11. Un n-uplet $\left(\Gamma_{j}\right)$ est pseudo-régulier si et seulement si les holonomies le long des cycles de mêmes longueurs coïncident.

Preuve. Supposons $\left(\Gamma_{j}\right)$ pseudo-régulier et notons $\gamma_{1}=\left(i_{1} \ldots i_{k}\right)$ et $\gamma_{2}=\left(j_{1} \ldots j_{k}\right)$ deux cycles de même longueur $k \in\{3, \ldots, n\}$. On sait qu'il existe une permutation $\sigma \in \mathcal{S}_{n}$ telle que $\sigma \gamma_{1} \sigma^{-1}=\gamma_{2}$. Puisque ( $\left.\Gamma_{j}\right)$ est pseudo-régulier, l'holonomie le long de $\gamma_{1}$ pour le $n$-uplet $\left(\Gamma_{j}\right)$ est égale à l'holonomie le long de $\gamma_{1}$ pour $\left(\Gamma_{\sigma(j)}\right)$, qui précisément est égale à l'holonomie le long de $\gamma_{2}$ pour $\left(\Gamma_{j}\right)$.

Réciproquement, supposons que les holonomies le long des cycles de même longueur coïncident. Soient $\sigma \in \mathcal{S}_{n}$ et $\gamma$ un cycle de $\mathcal{S}_{n}$. Alors $\sigma \gamma \sigma^{-1}$ est un cycle de même longueur, donc $h(\gamma)=h\left(\sigma \gamma \sigma^{-1}\right)$, or $h\left(\sigma \gamma \sigma^{-1}\right)$ représente l'holonomie le long de $\gamma$ pour le $n$-uplet $\left(\Gamma_{\sigma(j)}\right)$; ainsi $\left(\Gamma_{j}\right)$ est pseudo-régulier.

Pour terminer cette partie, nous étudions la régularité d'un type particulier de $n$-uplets. Etant donné un $n$-uplet $\left(\Gamma_{j}\right)$ admettant une matrice de Seidel associée $M$, que l'on suppose réduite, une condition nécessaire pour que $\left(\Gamma_{j}\right)$ soit régulier, qui se lit directement sur la matrice $M$, est que ses blocs non nuls $A_{i j}$ soient tous conjugués. En effet, la classe de conjugaison de $A_{i j}$ est l'holonomie le long du cycle ( $1 \mathrm{ij}$ ).

Le type particulier qui nous intéresse est lorsque tous les blocs $A_{i j}$ avec $2 \leq i<j \leq$ $n$ coïncident. Puisque les deux matrices de Seidel

$$
M(A, \ldots, A)=\left(\begin{array}{ccccc}
0 & I & I & \ldots & I \\
I & 0 & A & \ldots & A \\
I & { }^{t} A & \ddots & \ddots & \vdots \\
\vdots & \vdots & \ddots & \ddots & A \\
I & { }^{t} A & \ldots & { }^{t} A & 0
\end{array}\right) \quad \text { et } M_{A}=\left(\begin{array}{cccc}
0 & A & \ldots & A \\
{ }^{t} A & 0 & \ddots & \vdots \\
\vdots & \ddots & \ddots & A \\
{ }^{t} A & \ldots & { }^{t} A & 0
\end{array}\right)
$$

sont équivalentes (poser $P_{1}=A$ et $P_{2}=\cdots=P_{n}=I$ dans la définition 2.5), cela revient à étudier la régularité des $n$-uplets ayant une matrice de Seidel associée du type $M_{A}$. Pour $n=3$, nous verrons dans la partie 3 que tous les triplets sont réguliers, donc aucune condition sur $A$ n'apparait. En revanche, pour $n \geq 4$, seules les involutions conduisent à de tels $n$-uplets réguliers, comme le montre l'énoncé suivant.

Proposition 2.12. Soit $\left(\Gamma_{j}\right)$ un n-uplet avec $n \geq 4$, admettant une matrice de Seidel associée de type $M_{A}$ décrite dans (9), avec $A \in \mathcal{O}_{d}$. Alors les assertions suivantes sont équivalentes.

(i) $\left(\Gamma_{j}\right)$ est pseudo-régulier,

(ii) A est une involution de $\mathcal{O}_{d}$, i.e. $A^{2}=I$,

(iii) $\left(\Gamma_{j}\right)$ est régulier. 
Preuve. L'implication (i) $\Rightarrow$ (ii) s'obtient en comparant les holonomies le long des cycles (1234) et (1324), qui doivent coïncider d'après la proposition 2.11. On a

$$
h((1234))=\operatorname{cl}\left(A_{12} A_{23} A_{34} A_{41}\right)=\operatorname{cl}\left(A A A^{t} A\right)=\operatorname{cl}\left(A^{2}\right)
$$

et $h((1324))=\operatorname{cl}\left(A^{t} A A^{t} A\right)=\{I\}$, d'où $A^{2}=I$.

Montrons à présent que (ii) implique (iii). Puisque ${ }^{t} A=A^{-1}=A$, la matrice $M_{A}$ est de la forme

$$
M_{A}=\left(\begin{array}{cccc}
0 & A & \ldots & A \\
A & 0 & \ddots & \vdots \\
\vdots & \ddots & \ddots & A \\
A & \ldots & A & 0
\end{array}\right)
$$

Soit $\tau=(k l)$ une transposition de $\mathcal{S}_{n}$. On obtient une matrice de Seidel associée au $n$-uplet $\left(\Gamma_{\tau(j)}\right)$ en échangeant les $k$-ièmes et $l$-ièmes lignes et colonnes blocs de $M$; or cet échange n'a pas modifié $M$. On conclut avec le fait que $\mathcal{S}_{n}$ est engendré par les transpositions.

L’implication (iii) $\Rightarrow$ (i) est évidente.

Remarque. Pour $n \geq 3$ et pour chaque $d \geq 1$, la proposition 2.12 produit ainsi $d+1$ familles à un paramètre $c$, notées $\left\{\left(\Gamma_{j}(p, c)\right)_{1 \leq j \leq n}\right\}$, de $n$-uplets réguliers. En effet, les involutions de $\mathcal{O}_{d}$ sont exactement les matrices conjuguées à une matrice de la forme $\operatorname{Diag}\left(I_{d-p},-I_{p}\right)$ avec $0 \leq p \leq d$. Le calcul du spectre de $M_{A}$ fait dans la prochaine section 2.5 montre que la plus petite valeur propre $\mu_{0}$ de $M_{A}$ est $: \mu_{0}=1-n$, de multiplicité $p$ si $p>0$, et $\mu_{0}=-1$, de multiplicité $(n-1) d$ si $p=0$. D'après l'item 3 du théorème 2.3 , on a donc :

- Si $p>0$ alors $\left(\Gamma_{j}(p, c)\right)$ existe si et seulement si $c \leq \frac{1}{n-1}$. L'ordre de platitude est nul si $c<\frac{1}{n-1}$, égal à $p$ si $c=\frac{1}{n-1}$.

- Si $p=0$ alors $\left(\Gamma_{j}(p, c)\right)$ existe pour tout $c \leq 1$, d'ordre de platitude nul si $c<1$, égal à $(n-1) d$ pour le $n$-uplet trivial où $c=1$.

2.5 Produit tensoriel. Dans la section précédente, nous avons considéré des matrices $M_{A}$ données par (10), où $A$ est une matrice orthogonale involutive, donc conjuguée à $\operatorname{Diag}\left(I_{d-p},-I_{p}\right)$ pour un certain $p \in\{0, \ldots, d\}$. Si l'on note

$$
J_{n}=\left(\begin{array}{cccc}
0 & 1 & \ldots & 1 \\
1 & 0 & \ddots & \vdots \\
\vdots & \ddots & \ddots & 1 \\
1 & \ldots & 1 & 0
\end{array}\right)
$$

on a $M_{A}=J_{n} \otimes A$. Il est connu que les valeurs propres d'un produit tensoriel $B \otimes A$ sont les produits des valeurs propres de $A$ et de $B$. Ici le calcul du spectre de $J_{n}$ est très classique : les valeurs propres sont $\lambda_{1}=-1$, de multiplicité $n-1$, et $\lambda_{2}=n-1$ 
de multiplicité 1. Les valeurs propres de $M_{A}$ sont donc, par ordre croissant (en ignorant celles de multiplicité 0 lorsque $p=0$ ou $d): \mu_{0}=1-n$, de multiplicité $p, \mu_{1}=-1$ de multiplicité $(n-1)(d-p), \mu_{2}=1$ de multiplicité $(n-1) p$ et $\mu_{3}=n-1$ de multiplicité $d-p$. Ceci justifie ce qui était annoncé à la fin de la section précédente et de la section 2.2.

L'objet de cette section est de présenter d'autres constructions de matrices de Seidel par produit tensoriel. Une motivation pour ces constructions est la suivante. L'étude des droites équiangulaires a été amorcée parallèlement par J. Haantjes [10] et L.M. Blumenthal en 1947, et poursuivie par J.H. van Lint et J.J. Seidel en 1965 et par P.W.H. Lemmens et J.J. Seidel en 1973 [16] et [13]. Pour plus de détails, c.f. [14]. Nous verrons que la résolution du problème des droites a des conséquences pour le problème des sous-espaces.

Appelons B-matrice (resp. B'-matrice) une matrice carrée symétrique (resp. antisymétrique) ayant une diagonale de zéros et des \pm 1 ailleurs. La notion de B-matrice a été introduite dans [16] et celle de B'-matrice dans [6]. On dira que deux B-matrices ou deux B'-matrices $B_{1}$ et $B_{2}$ d'ordre $n$ sont équivalentes s'il existe une matrice $C$ de la forme $C=\operatorname{Diag}\left(\varepsilon_{1}, \ldots, \varepsilon_{n}\right)$ avec $\varepsilon_{i} \in\{-1,1\}$ telle que $B_{2}=C B_{1} C$. Autrement dit, deux B-matrices (resp. B'-matrices) sont équivalentes si et seulement si l'on peut passer de l'une à l'autre par une succession d'opérations élémentaires qui consistent à multiplier une ligne et la colonne correspondante par -1 .

Etant donnée une matrice symétrique $A \in \mathcal{O}_{d}$ et une B-matrice $B$, le produit tensoriel $B \otimes A$ est une matrice de Seidel. Il en est de même du produit tensoriel d'une matrice antisymétrique de $\mathcal{O}_{d}$ par une B'-matrice.

Notons que, si deux B-matrices (resp. B'-matrices) $B_{1}$ et $B_{2}$ sont équivalentes, alors pour toute matrice symétrique (resp. antisymétrique) $A \in \mathcal{O}_{d}$, les matrices de Seidel $M_{1}=B_{1} \otimes A$ et $M_{2}=B_{2} \otimes A$ sont équivalentes au sens de la définition 2.5. En effet, on a $B_{2} \otimes A={ }^{t} D\left(B_{1} \otimes A\right) D$ avec $D=\operatorname{Diag}\left(\varepsilon_{1} I, \ldots, \varepsilon_{n} I\right)$.

Soit à présent $B$ une $\mathrm{B}$-matrice et $A_{1}, A_{2}$ deux matrices symétriques de $\mathcal{O}_{d}$ (resp. $B$ une B'-matrice et $A_{1}, A_{2} \in \mathcal{O}_{d}$ antisymétriques). Alors une condition nécessaire et suffisante pour que les deux matrices de Seidel $M_{1}=B \otimes A_{1}$ et $M_{2}=B \otimes A_{2}$ soient équivalentes au sens de la définition 2.5 est que les matrices $A_{1}$ et $A_{2}$ soient conjuguées. En effet, la condition est clairement suffisante, et pour montrer qu'elle est nécessaire il suffit d'utiliser les formes réduites de $M_{1}$ et $M_{2}$, d'utiliser la remarque précédente et le corollaire 2.7.

Dans $\mathcal{O}_{d}$, les matrices symétriques se réduisent aux matrices involutives, i.e. satisfaisant $A^{2}=I$, et les matrices antisymétriques se réduisent aux matrices anti-involutives, i.e. satisfaisant $A^{2}=-I$.

Etant donné un $n$-uplet $\left(\Gamma_{j}\right)$ de paramètre $c$ de $G(d, N)$, P.W.H. Lemmens et J.J. Seidel donnent dans [12] une condition nécessaire et suffisante en termes de projecteurs associés pour l'existence de $n$ droites réelles $\delta_{1} \subset \Gamma_{1}, \ldots, \delta_{n} \subset \Gamma_{n}$ qui sont équi-angulaires avec l'angle $\arccos c$. Par ailleurs dans [7] on trouve, pour le cas $d=2$, une condition nécessaire et suffisante en termes de matrices de Seidel associées pour l'existence de ces $n$ droites. Dans la proposition qui suit, nous donnons une condition suffisante en dimension $d$ quelconque et nous considérons aussi le cas des plans.

Proposition 2.13. 1. Etant donné un n-uplet $\left(\Gamma_{j}\right)$ de paramètre $c$ et de matrice associée $B \otimes A$ où $A$ est une involution et $B$ est une B-matrice, il existe $n$ droites réelles $\delta_{1} \subset$ 
$\Gamma_{1}, \ldots, \delta_{n} \subset \Gamma_{n}$ qui sont équi-angulaires avec l'angle $\arccos c$.

2. Etant donné un n-uplet $\left(\Delta_{j}\right)$ de paramètre $c$ et de matrice associée $B \otimes A$ où $A$ est une anti-involution et $B$ est une $B$ '-matrice, il existe n plans réels $\pi_{1} \subset \Delta_{1}, \ldots, \pi_{n} \subset \Delta_{n}$ qui sont de même paramètre $c$.

Preuve. 1. Soit $\left(\Gamma_{j}\right)$ un tel $n$-uplet et $M=B \otimes A$ la matrice associée donnée par l'énoncé. Il existe $P \in \mathcal{O}_{d}$ tel que $P A P^{-1}=A^{\prime}:=\operatorname{Diag}\left(I_{d-p},-I_{p}\right)$. Ainsi $M$ est équivalente à la matrice de Seidel $M^{\prime}:=B \otimes A^{\prime}$, ce qui signifie qu'il existe une base orthonormée $\left(u_{j}^{1}, \ldots, u_{j}^{d}\right)$ dans chaque $\Gamma_{j}$ telle que la matrice de Gram de $\left(u_{1}^{1}, \ldots, u_{1}^{d}, \ldots, u_{n}^{1}\right.$, $\left.\ldots, u_{n}^{d}\right)$ soit $I+c M^{\prime}$. En supprimant les $d-1$ dernières lignes de chaque bloc de $d$ lignes et les colonnes correspondantes, on obtient la matrice de Gram associée au $n$-uplet des droite $\delta_{1}, \ldots, \delta_{n}$ engendrées respectivement par $u_{1}^{1}, \ldots, u_{n}^{1}$. Ces droites sont bien équiangulaires d'angle $\arccos c$.

2. Soit $\left(\Delta_{j}\right)$ un tel $n$-uplet et $M=B \otimes A$ la matrice associée donnée par l'énoncé. A présent $A$ est une anti-involution, donc d'une part $d$ est pair et d'autre part il existe $P \in \mathcal{O}_{d}$ tel que $P A P^{-1}=A^{\prime}:=\left(\begin{array}{cc}0 & -I_{p} \\ I_{p} & 0\end{array}\right)$, avec $2 p=d$. Ainsi $M$ est équivalente à la matrice de Seidel $M^{\prime}:=B \otimes A^{\prime}$ : il existe donc une base orthonormée $\left(u_{j}^{1}, \ldots, u_{j}^{d}\right)$ dans chaque $\Gamma_{j}$ telle que $\operatorname{Gram}\left(u_{1}^{1}, \ldots, u_{1}^{d}, \ldots, u_{n}^{1}, \ldots, u_{n}^{d}\right)=I+c M^{\prime}$.

En supprimant de la deuxième à la $p$-ième ligne et de la $(p+2)$-ième à la $(2 p)$ ième ligne de chaque bloc ligne et les colonnes correspondantes, autrement dit en ne conservant que les lignes et colonnes 1 et $p+1$ de chaque bloc, on obtient $I+c(B \otimes R)$ comme matrice de Gram, avec $R:=R_{\pi / 2}=\left(\begin{array}{cc}0 & -1 \\ 1 & 0\end{array}\right)$. On vérifie que les plans $\pi_{1}=$ $\left\langle u_{1}^{1}, u_{1}^{p+1}\right\rangle, \ldots, \pi_{n}=\left\langle u_{n}^{1}, u_{n}^{p+1}\right\rangle$ répondent à la question.

Remarques. 1. Il est montré dans [8] que les plans obtenus précédemment peuvent être vus comme des droites complexes équi-angulaires avec l'angle $\arccos c$, c.f. aussi la proposition 5.1.

2. Dans [16], les auteurs représentent une B-matrice $\left(b_{i j}\right)_{1 \leq i, j \leq n}$ d'ordre $n$ par un $n$-graphe de la façon suivante : on considère un $n$-uplet de points numérotés de 1 à $n$ et l'on joint une paire $\{i, j\}$ par une arête si et seulement si $b_{i j}=-1$. Les opérations élémentaires sur les B-matrices se traduisent alors sur les graphes de la manière suivante : en un sommet arbitraire du graphe, enlever les arêtes existantes et rajouter celles qui n'existaient pas. Deux graphes sont dits équivalents si l'on peut passer de l'un à l'autre par ces opérations élémentaires. Il est alors montré qu'il y a une bijection entre les classes de congruence de $n$-uplets de droites équi-angulaires et les classes d'équivalence de $n$ graphes. Deux classes d'équivalence de graphes $G_{1}$ et $G_{2}$ sont dites complémentaires s'il existe deux B-matrices $B_{1}$ et $B_{2}$ associées respectivement à $G_{1}$ et $G_{2}$ telles que $B_{1}=$ $-B_{2}$, autrement dit si ces classes ont des graphes représentatifs complémentaires. Les $n$-graphes auto-complémentaires sont indiqués dans [16] pour tout $n \in\{2, \ldots, 7\}$.

A deux classes de $n$-graphes complémentaires $G_{1}$ et $G_{2}$ sont associées respectivement deux B-matrices $B_{1}$ et $B_{2}$ de spectres opposés. Pour $d$ pair et pour $p=\frac{d}{2}$, les produits 
$M_{j}=B_{j} \otimes A$, avec $A:=\operatorname{Diag}\left(I_{p},-I_{p}\right)$ et $j=1$ ou 2 , fournissent deux matrices de Seidel équivalentes : appliquer le corollaire 2.7 avec $P=\left(\begin{array}{cc}0 & -I_{p} \\ I_{p} & 0\end{array}\right)$.

Si de plus $B$ provient d'un graphe auto-complémentaire, alors le spectre de $B$ est symétrique par rapport à 0 . Par conséquent les matrices $M_{0}, \ldots, M_{d}$ données par $M_{p}=$ $B \otimes \operatorname{Diag}\left(I_{d-p},-I_{p}\right)$, bien que non équivalentes au sens de la définition 2.5 , ont même spectre.

\section{Les triplets}

Rappelons que le mot "isoclin" est systématiquement sous-entendu. Soit $\left(\Gamma_{1}, \Gamma_{2}, \Gamma_{3}\right)$ un triplet de $G(d, 3 d)$ et $M(A)$ une forme réduite, $A \in \mathcal{O}_{d}$. On rappelle la notation

$$
M(A):=\left(\begin{array}{ccc}
0 & I & I \\
I & 0 & A \\
I & { }^{t} A & 0
\end{array}\right) .
$$

On sait que toute matrice orthogonale $A \in \mathcal{O}_{d}$ est semblable à une matrice diagonale par blocs de la forme

$$
\operatorname{Diag}\left(R_{\omega_{1}}, \ldots, R_{\omega_{m}}, I_{p},-I_{q}\right),
$$

où $p$ et $q \in\{0, \ldots, d\}$ sont respectivement les multiplicités de 1 et de -1 comme valeurs propres éventuelles de $A, m=\frac{1}{2}(d-p-q) \in\left[0, \frac{d}{2}\right]$ et $R_{\omega_{j}}=\left(\begin{array}{cc}\cos \omega_{j} & -\sin \omega_{j} \\ \sin \omega_{j} & \cos \omega_{j}\end{array}\right)$, $0<\omega_{1} \leq \cdots \leq \omega_{m}<\pi$.

Puisque $p, q$ et $\omega_{1}, \ldots, \omega_{m}$ sont des invariants par similitude de $A$, ils ne dépendent pas du choix de la forme réduite choisie, i.e. du choix de la base orthonormée choisie dans $\Gamma_{1}$ pour déterminer les bases dans $\Gamma_{2}$ et $\Gamma_{3}$. Ils ne dépendent donc que du triplet $\left(\Gamma_{1}, \Gamma_{2}, \Gamma_{3}\right)$.

Appelons $(p, q)$ la signature du triplet $\left(\Gamma_{1}, \Gamma_{2}, \Gamma_{3}\right)$ et $\omega_{1}, \ldots, \omega_{m}$ les angles de $\left(\Gamma_{1}\right.$, $\left.\Gamma_{2}, \Gamma_{3}\right)$.

Proposition 3.1. 1. Deux triplets de même paramètre c sont congruents si, et seulement si, ils ont même signature $(p, q)$ et mêmes angles $\omega_{1}, \ldots, \omega_{m}$.

\section{Un triplet est toujours régulier.}

3. La classe de congruence d'un triplet est déterminée par son holonomie le long d'un des 3-cycles.

Preuve. 1. D'après le corollaire 2.7, deux triplets de même paramètre $c$ sont congruents si, et seulement si, ils ont des formes réduites $M(A)$ et $M(\widetilde{A})$ avec des matrices $A$ et $\widetilde{A}$ conjuguées, ce qui équivaut au fait que les triplets ont mêmes signatures et mêmes angles.

2. Le groupe $\mathcal{S}_{3}$ étant engendré par les transpositions (12) et (23), il suffit de vérifer que la forme réduite obtenue après ces deux échanges de sommets $\Gamma_{j}$ satisfait la condition 
du corollaire 2.7. Une permutation de $\Gamma_{2}$ et $\Gamma_{3}$ transforme $M(A)$ en $M\left({ }^{t} A\right)$. Une permutation de $\Gamma_{1}$ et $\Gamma_{2}$ transforme $M(A)$ en $\left(\begin{array}{ccc}0 & I & A \\ I & 0 & I \\ { }^{t} A & I & 0\end{array}\right)$ qui, une fois mise sous forme réduite par multiplication de la dernière ligne par $A$ et de la dernière colonne par ${ }^{t} A$, donne $M\left({ }^{t} A\right)$. On conclut avec le fait que ${ }^{t} A$ est conjuguée à $A$.

3. Dans des bases adaptées, $c^{-3} \pi_{12} \circ \pi_{23} \circ \pi_{31}$ a pour matrice $A$ et $c^{-3} \pi_{13} \circ \pi_{32} \circ \pi_{21}$ a pour matrice ${ }^{t} A$, qui est conjuguée à $A$. Ainsi, l'holonomie le long de tout 3-cycle est $\operatorname{cl}(A)$ qui, d'après le point 1 , détermine le triplet à isométrie de $\mathbb{R}^{N}$ près.

Remarque. Lorsque $d=2$ et $A$ est une matrice de rotation d'angle $\omega$, nous retrouvons l'invariant de forme introduit par U. Brehm [2]. En effet, comme nous le verrons dans la proposition 5.1, le triplet est en bijection canonique avec un triplet équilatère de droites complexes dont des vecteurs directeurs unitaires ont une matrice de Gram de la forme $I+c H$ avec $H=\left(\begin{array}{ccc}0 & 1 & 1 \\ 1 & 0 & e^{i \omega} \\ 1 & e^{-i \omega} & 0\end{array}\right)$. Le triplet est donc déterminé à isométrie près par son paramètre $c$ et son invariant de forme $\cos \omega$.

Le résultat suivant a déjà été énoncé dans [7] dans le cas $d=2$.

Théorème 3.2. Soit $\left(\Gamma_{1}, \Gamma_{2}, \Gamma_{3}\right)$ un triplet de $G(d, 3 d)$ de paramètre $\left.\left.c \in\right] 0,1\right]$, de signature $(p, q)$ et d'angles $\omega_{1}, \ldots, \omega_{m}$ avec $p, q, \omega_{j} \geq 0$ et $p+q+2 m=d$.

Si $q \neq 0$, alors l'ordre de platitude du triplet est égal à 0 si $c<\frac{1}{2}$, et égal à $q$ si $c=\frac{1}{2}$.

Sinon, soit $r \in\{1, \ldots, m\}$ tel que $0<\omega_{1} \leq \cdots \leq \omega_{m-r}<\omega_{m-r+1}=\cdots=\omega_{m}<\pi$ (avec la convention $\omega_{0}=0$ dans le cas $r=m$ ). Alors l'ordre de platitude du triplet est 0 si $c<-\frac{1}{\mu_{0}}$ et $2 r$ si $c=-\frac{1}{\mu_{0}}$, où $\mu_{0}=2 \cos \left(\frac{\omega_{m}+2 \pi}{3}\right)$.

Preuve. Soit $M(A)=\left(\begin{array}{ccc}0 & I & I \\ I & 0 & A \\ I & { }^{t} A & 0\end{array}\right)$ une forme réduite de $\left(\Gamma_{1}, \Gamma_{2}, \Gamma_{3}\right)$; ainsi $A$ est semblable à $\operatorname{Diag}\left(R_{\omega_{1}}, \ldots, R_{\omega_{m}}, I_{p},-I_{q}\right)$. Pour $x \in \mathbb{R}$, notons $X:=x I_{d}$. Ainsi le polynôme caractéristique de $M(A)$ est le déterminant de la matrice

$$
B:=\left(\begin{array}{ccc}
X & -I & -I \\
-I & X & -A \\
-I & -{ }^{t} A & X
\end{array}\right) .
$$

Puisque les blocs de $B$ commutent, son déterminant se calcule en deux temps par un procédé classique. Pour la complétude de l'article, nous détaillons ce procédé ci-dessous. Pour un énoncé général concernant ces calculs de déterminants, on peut consulter [15].

Considérons la matrice suivante :

$$
C:=\left(\begin{array}{lll}
X^{2}-I & 0 & 0 \\
A+X & I & 0 \\
{ }^{t} A+X & 0 & I
\end{array}\right)
$$


Etant une matrice triangulaire par blocs, son déterminant satisfait $\operatorname{det} C=\operatorname{det}\left(X^{2}-I\right)$. Le calcul par blocs du produit $B C$, utilisant $A X=X A$ et $A^{t} A=I$, donne

$$
B C=\left(\begin{array}{ccc}
X^{3}-3 X-\left(A+{ }^{t} A\right) & -I & -I \\
0 & X & -A \\
0 & -{ }^{t} A & X
\end{array}\right) .
$$

De la même façon, on a $\left(\begin{array}{cc}X & -A \\ -{ }^{t} A & X\end{array}\right)\left(\begin{array}{ll}X & 0 \\ { }^{t} A & I\end{array}\right)=\left(\begin{array}{cc}X^{2}-I & -A \\ 0 & X\end{array}\right)$. En comparant les différents déterminants de ces matrices, on obtient ainsi :

$$
P_{M(A)}(x)=\operatorname{det} B=\operatorname{det}\left(X^{3}-3 X-\left(A+{ }^{t} A\right)\right) .
$$

Le calcul donne alors

$$
P_{M(A)}(x)=\left(x^{3}-3 x-2\right)^{p}\left(x^{3}-3 x+2\right)^{q} \prod_{j=1}^{m}\left(x^{3}-3 x-2 \cos \omega_{j}\right)^{2}
$$

donc les valeurs propres de $M(A)$ sont égales à :

- $2 \cos \left(\frac{\omega_{j}+2 k \pi}{3}\right), j=1, \ldots, m, k=0,1$ ou 2 si $m \neq 0$,

- -1 de multiplicité $2 p$ et 2 de multiplicité $p$ si $p \neq 0$,

- 1 de multiplicité $2 q$ et -2 de multiplicité $q$ si $q \neq 0$.

Remarquons que, puisque $\omega_{1}, \ldots, \omega_{m}$ sont compris entre 0 et $\pi$, on a $0<\frac{\omega_{j}}{3}<\frac{\pi}{3}$, $\frac{2 \pi}{3}<\frac{\omega_{j}+2 \pi}{3}<\pi$ et $\frac{4 \pi}{3}<\frac{\omega_{j}+4 \pi}{3}<\frac{5 \pi}{3}$, donc parmi les angles $\frac{\omega_{j}+2 k \pi}{3}, j=1, \ldots, m$, $k=0,1$ ou 2 , c'est l'angle $\frac{\omega_{m}+2 \pi}{3}$ qui a le plus petit cosinus.

Si $q \neq 0$, alors la plus petite valeur propre $\mu_{0}$ de $M(A)$ est -2 , de multiplicité $q$; si $q=0$ alors $\mu_{0}=2 \cos \left(\frac{\omega_{m}+2 \pi}{3}\right)$, de multiplicité $2 r$. On conclut avec le théorème 2.3, item 3 .

\section{Les quadruplets}

Soit $\left(\Gamma_{1}, \Gamma_{2}, \Gamma_{3}, \Gamma_{4}\right)$ un quadruplet de $G(d, 4 d)$. Pour simplifier la suite, nous choisissons une forme réduite de ce quadruplet sous la forme $M\left(A,{ }^{t} C, B\right), A, B, C \in \mathcal{O}_{d}$ :

$$
M\left(A,{ }^{t} C, B\right):=\left(\begin{array}{cccc}
0 & I & I & I \\
I & 0 & A & { }^{t} C \\
I & { }^{t} A & 0 & B \\
I & C & { }^{t} B & 0
\end{array}\right) .
$$

A présent les holonomies sont au nombre de $7:$ ce sont respectivement les classes de conjugaison de $A$ pour les cycles (123) et (132), $B$ pour les cycles (134) et (143), $C$ pour les cycles (124) et (142), $A B C$ pour les cycles (234) et (243), $A B$ pour les cycles (1234) et (1432), $B C$ pour les cycles (1243) et (1342), et $C A$ pour les cycles (1324) et (1423). La proposition 2.11 entraîne immédiatement le résultat suivant. 


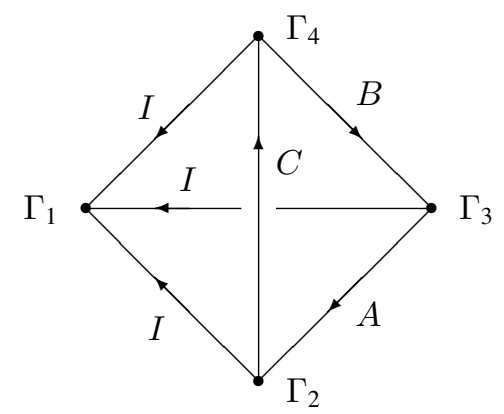

FIGURE 1. Les matrices des isométries obtenues par projection orthogonale suivie de l'homothétie de rapport $\frac{1}{c}$.

Proposition 4.1. Soit $\left(\Gamma_{1}, \Gamma_{2}, \Gamma_{3}, \Gamma_{4}\right)$ un quadruplet de $G(d, 4 d)$ de forme réduite $M(A$, $\left.{ }^{t} C, B\right), A, B, C \in \mathcal{O}_{d}$. Ce quadruplet est pseudo-régulier si et seulement si les quatre matrices $A, B, C, A B C$ sont conjuguées et les trois matrices $A B, B C, C A$ sont conjuguées.

Jusqu'à présent nous n'avons considéré que des $n$-uplets ordonnés, mais par la suite nous établirons des résultats où l'ordre n'importe pas. Le résultat qui suit nous servira dans la prochaine partie à réduire le nombre de cas à examiner.

Proposition 4.2. Etant donné un quadruplet $\left(\Gamma_{1}, \Gamma_{2}, \Gamma_{3}, \Gamma_{4}\right)$, il existe une permutation $\sigma \in \mathcal{S}_{4}$ telle $\left(\Gamma_{j}\right)$ ait une forme réduite $M\left(A,{ }^{t} C, B\right)$ de l'un des trois types suivants : type I : $A, B, C \in \mathcal{O}_{d}^{+}$, type II : $A, B, C \in \mathcal{O}_{d}^{-}$, type III : $A, B \in \mathcal{O}_{d}^{+}, C \in \mathcal{O}_{d}^{-}$.

Preuve. Pour faire court, nous appelons 3-holonomie une holonomie le long d'un 3-cycle. Une permutation des sommets $\left(\Gamma_{j}\right)$ induit une permutation sur les holonomies. Commençons par montrer qu'on peut obtenir toute permutation sur les 3-holonomies par une permutation des sommets $\left(\Gamma_{j}\right)$.

Pou cela, notons $a=\operatorname{cl}(A), b=\operatorname{cl}(B), c=\operatorname{cl}(C)$ et $d=\operatorname{cl}(A B C)$ les 3-holonomies de $\left(\Gamma_{1}, \Gamma_{2}, \Gamma_{3}, \Gamma_{4}\right)$ et considérons l'application

$$
p: \mathcal{S}(\{1,2,3,4\}) \rightarrow \mathcal{S}(\{a, b, c, d\}), \sigma \mapsto s=p(\sigma)
$$

telle que les 3-holonomies de $\left(\Gamma_{\sigma(j)}\right)$ soient dans l'ordre $s(a), s(b), s(c), s(d)$. Cette application est un morphisme injectif d'ensembles de même cardinal ; elle est donc surjective.

Par ailleurs, puisque la quatrième 3-holonomie $d$ est la classe du produit $A B C$, le nombre de 3-holonomies qui sont des classes de conjugaison dans $\mathcal{O}_{d}^{-}$est nécessairement pair. Le type I correspond au cas où les quatre 3-holonomies sont des classes dans $\mathcal{O}_{d}^{+}$, le type II où les quatre sont dans $\mathcal{O}_{d}^{-}$, et le type III au cas où deux sont dans $\mathcal{O}_{d}^{+}$et deux dans $\mathcal{O}_{d}^{-}$, et où par une permutation on s'est ramené à $a, b \subset \mathcal{O}_{d}^{+}$.

Remarques. 1. Notons que la surjectivité de l'application $p$ est spécifique du cas $n=4$. A partir de $n=5$, le nombre de 3-holonomies, égal à $\left(\begin{array}{l}n \\ 3\end{array}\right)$, est strictement supérieur à $n$, donc l'application analogue de $p$ ne peut pas être surjective. 


\begin{tabular}{|c|c|c|}
\hline$\sigma \in \mathcal{S}_{4}$ & Matrice de Seidel & $s=p(\sigma) \in \mathcal{S}(\{a, b, c, d\})$ \\
\hline id & $M\left(A,{ }^{t} C, B\right)$ & id \\
$(12)$ & $M\left({ }^{t} A, C, A B C\right)$ & $(b d)$ \\
$(13)$ & $M\left({ }^{t} A,{ }^{t}(B C A),{ }^{t} B\right)$ & $(c d)$ \\
$(14)$ & $M\left(C A B, C,{ }^{t} B\right)$ & $(a d)$ \\
$(23)$ & $M\left({ }^{t} A, B,{ }^{t} C\right)$ & $(b c)$ \\
$(24)$ & $M\left({ }^{t} B, C,{ }^{t} A\right)$ & $(a b)$ \\
$(34)$ & $M\left({ }^{t} C, A,{ }^{t} B\right)$ & $(a c)$ \\
\hline
\end{tabular}

TABLE 1. Formes réduites et action sur les holonomies après transposition de sommets.

2. Nous aurons besoin par la suite de connaître une matrice de Seidel associée au quadruplet après permutation des sommets. Dans la table 1, nous présentons l'action des transpositions. Nous nous sommes restreints aux transpositions dans un souci de concision. Nous y avons joint la permutation induite sur les 3-holonomies, ce qui permet de confirmer la surjectivité de $p$.

Détaillons sur l'exemple de la transposition (12) comment est construite cette table. Une permutation de $\Gamma_{1}$ et $\Gamma_{2}$ conduit à permuter les blocs lignes et colonnes 1 et 2 de la matrice de Seidel $M\left(A,{ }^{t} C, B\right)$, i.e. à la matrice

$$
\left(\begin{array}{llll}
0 & I & A & { }^{t} C \\
I & 0 & I & I \\
{ }^{t} A & I & 0 & B \\
C & I & { }^{t} B & 0
\end{array}\right) .
$$

Pour ramener cette nouvelle matrice de Seidel à une forme réduite, i.e. avec des blocs $I$ sur la première ligne et la première colonne, on multiplie la ligne 3 par $A$, la colonne 3 par ${ }^{t} A$, la ligne 4 par ${ }^{t} C$ et la colonne 4 par $C$; autrement dit, on effectue des changements de bases dans les sous-espaces $\Gamma_{3}$ et $\Gamma_{4}$ de matrices respectivement $A$ et $C$. Ainsi le quadruplet $\left(\Gamma_{2}, \Gamma_{1}, \Gamma_{3}, \Gamma_{4}\right)$ admet pour forme réduite $M\left({ }^{t} A, C, A B C\right)$. Puisque $A$ est conjuguée à ${ }^{t} A$ et $C$ à ${ }^{t} C$, la permutation des sommets $\Gamma_{1}$ et $\Gamma_{2}$ induit ainsi une permutation des 3-holonomies $b$ et $d$; en d'autres termes, on a $p((12))=(b d)$.

3. En généralisant cette classification des quadruplets aux $n$-uplets, nous proposons la définition suivante.

Définition 4.3. Un $n$-uplet est dit de type I si toutes ses 3-holonomies sont dans $\mathcal{O}_{d}^{+}$. Il est de type II si toutes ses 3 -holonomies sont dans $\mathcal{O}_{d}^{-}$. Il est d'un type mixte s'il contient des 3-holonomies dans $\mathcal{O}_{d}^{+}$et dans $\mathcal{O}_{d}^{-}$.

Revenons à présent à la question de savoir si les holonomies suffisent ou non à déterminer la classe de congruence du $n$-uplet. Nous verrons dans la prochaine partie que la réponse est positive en dimension deux. L'exemple suivant montre que la réponse est négative en dimension supérieure. 
Exemple. Soit $\left(\Gamma_{1}, \Gamma_{2}, \Gamma_{3}, \Gamma_{4}\right)$ et $\left(\widetilde{\Gamma}_{1}, \widetilde{\Gamma}_{2}, \widetilde{\Gamma}_{3}, \widetilde{\Gamma}_{4}\right)$ deux quadruplets de $G(3,12)$ de formes réduites $M\left(A,{ }^{t} C, B\right)$ et $M\left(\widetilde{A},{ }^{t} \widetilde{C}, \widetilde{B}\right)$ avec :

$$
\begin{gathered}
A=\left(\begin{array}{ccc}
0 & -1 & 0 \\
0 & 0 & -1 \\
1 & 0 & 0
\end{array}\right), \quad \widetilde{A}=\left(\begin{array}{ccc}
0 & 1 & 0 \\
0 & 0 & -1 \\
-1 & 0 & 0
\end{array}\right), \\
B=\widetilde{B}=\left(\begin{array}{ccc}
-1 & 0 & 0 \\
0 & -1 & 0 \\
0 & 0 & 1
\end{array}\right), \quad C=\widetilde{C}=\left(\begin{array}{lll}
0 & 0 & 1 \\
1 & 0 & 0 \\
0 & 1 & 0
\end{array}\right) .
\end{gathered}
$$

Rappelons que deux éléments de $\mathcal{O}_{3}^{+}$sont conjugués si, et seulement si, ils ont même angle. On vérifie que $A, \widetilde{A}, C, \widetilde{C}, A B, \widetilde{A} \widetilde{B}, B C$ et $\widetilde{B} \widetilde{C}$ sont des tiers de tours, donc tous conjugués, et que $B, \widetilde{B}, A B C, \widetilde{A} \widetilde{B} \widetilde{C}, C A$ et $\widetilde{C} \widetilde{A}$ sont des demi-tours, donc eux aussi conjugués. Ainsi les quadruplets $\left(\Gamma_{j}\right)$ et $\left(\widetilde{\Gamma}_{j}\right)$ ont mêmes holonomies. Pourtant ces quadruplets ne sont pas congruents. En effet un calcul simple montre que seules les matrices de la forme $k I$ peuvent conjuguer simultanément $B$ et $C$ à elles-mêmes, donc aucune ne peut conjuguer simultanément $A, B, C$ à $\widetilde{A}, \widetilde{B}, \widetilde{C}$.

Il existe aussi des quadruplets de $G(3,12)$ qui sont pseudo-réguliers mais non réguliers. Cependant leur construction dépasse le cadre du présent article.

Par ailleurs, nous n'avons considéré dans cet article que des holonomies dites primaires, mais en ajoutant un petit nombre $(\leq 4)$ d'holonomies le long d'autres lacets, on peut obtenir un système complet d'invariants par congruence de quadruplets de $G(3,12)$ et de $G(4,16)$. L'étude des $n$-uplets de sous-espaces de dimensions 3 et 4 fera l'objet d'un article ultérieur.

\section{Les $n$-uplets de plans}

Nous présentons dans cette partie deux critères de congruence de $n$-uplets de $G(2,2 n)$. Le premier, concernant les quadruplets, porte sur les holonomies. Le second critère porte sur les forme réduites. A la fin de cette partie, nous apportons des éléments de réponse à notre deuxième problème dans le cas des plans : quel est le nombre minimal $k$ tel qu'un $n$-uplet de plans est déterminé à congruence près par ses sous $k$-uplets ? Bien entendu, ce nombre $k$ est nécessairement supérieur à 3 , quelque soit son type : un tel $n$-uplet avec $n \geq 4$ ne peut être déterminé ni par ses sous-paires (qui sont déterminées à congruence près par le paramètre $c$ ) ni par ses sous-triplets (déterminés par leurs 3-holonomies). Il est donc naturel de se demander s'il est déterminé ou non par ses sous-quadruplets. La réponse est différente suivant le type du $n$-uplet. Elle est positive dans le cas du type I et du type II, mais négative dans le cas des types mixtes.

Notons les éléments de $\mathcal{O}_{2}^{+} \operatorname{par} R_{\varphi}=\left(\begin{array}{cc}\cos \varphi & -\sin \varphi \\ \sin \varphi & \cos \varphi\end{array}\right)$ et ceux de $\mathcal{O}_{2}^{-} \operatorname{par} S_{\varphi}=$ $\left(\begin{array}{cc}\cos \varphi & \sin \varphi \\ \sin \varphi & -\cos \varphi\end{array}\right)$. Les formules de produits sont

$$
R_{\alpha} R_{\beta}=R_{\alpha+\beta}, \quad R_{\alpha} S_{\beta}=S_{\alpha+\beta}, \quad S_{\alpha} R_{\beta}=S_{\alpha-\beta}, \quad S_{\alpha} S_{\beta}=R_{\alpha-\beta} .
$$


Tous nos angles sont des classes d'équivalence de nombres réels modulo $2 \pi$, sans que nous précisions mod $2 \pi$. Ainsi, par exemple $2 \varphi=0 \Leftrightarrow \varphi=0$ ou $\pi$. Deux rotations $R_{\alpha}$ et $R_{\beta}$ sont conjuguées si et seulement si $\alpha= \pm \beta$. Deux symétries sont toujours conjuguées.

A un $n$-uplet de plans de type I est associée une matrice de Seidel $M$ dont les blocs non nuls $A_{k l}$ sont tous des rotations. Notons $\omega_{k l}$ l'angle du bloc $A_{k l}$. A cette matrice $M$ est associée de manière canonique la matrice hermitienne $H=\left(h_{k l}\right)_{1 \leq k, l \leq n}$ avec $h_{k k}=0$ et pour $k \neq l, h_{k l}=e^{i \omega_{k l}}$. Dans [8], il est montré (en utilisant le résultat principal de [15]) que le polynôme caractéristique de $M$ est égal au carré de celui de $H$. La plus petite valeur propre $\mu_{0}$ de $M$ est donc paire et est aussi la plus petite valeur propre de $H$. Si $0<c \leq-\frac{1}{\mu_{0}}$, alors la matrice $I+c H$ est hermitienne positive. C'est donc la matrice de Gram de $n$ vecteurs de $\mathbb{C}^{n}$ engendrant $n$ droites équiangulaires de $\mathbb{C}^{n}$ d'angle $\arccos c$.

Réciproquement, tout $n$-uplet de droites équiangulaires de $\mathbb{C}^{n}$ peut être vu comme un $n$-uplet de plans de $\mathbb{R}^{2 n}$. Ainsi nous avons le résultat suivant.

Proposition 5.1. Les classes de congruence de n-uplets de plans de type I sont en bijection canonique avec les classes de congruence de n-uplets équilatères de droites complexes.

A présent, voici notre premier critère de congruence.

Théorème 5.2. Les holonomies et le paramètre c forment un système complet d'invariants par congruence de $G(2,8)$; autrement dit, deux quadruplets de $G(2,8)$ sont congruents si, et seulement si, ils ont même paramètre c et mêmes holonomies. En particulier, un quadruplet pseudo-régulier de $G(2,8)$ est forcément régulier.

Pour la preuve, nous aurons besoin du résultat suivant.

Lemme 5.3. Si $A, B, A^{\prime}, B^{\prime} \in \mathcal{O}_{2}$ sont telles que $A \sim A^{\prime}, B \sim B^{\prime}$ et $A B \sim A^{\prime} B^{\prime}$, alors $A$ et $B$ sont simultanément conjuguées à $A^{\prime}$ et $B^{\prime}$ : il existe $P \in \mathcal{O}_{2}$ telle que $A P=P A^{\prime}$ et $B P=P B^{\prime}$.

Preuve du lemme 5.3. On peut supposer sans perte de généralité que $A=A^{\prime}$. En effet, si $U$ conjugue $A$ à $A^{\prime}$ (i.e. $A U=U A^{\prime}$ ) $V$ conjugue $B$ à $B^{\prime}$ et $W$ conjugue $A B$ à $A^{\prime} B^{\prime}$, alors en posant $\widetilde{A}=A$ et $\widetilde{B}=U B^{\prime} U^{-1}, V U^{-1}$ conjugue $B$ à $\widetilde{B}$ et $W U^{-1}$ conjugue $A B$ à $\widetilde{A} \widetilde{B}$. Si dans ce cas particulier où $A=\widetilde{A}$ on en déduit une conjuguante commune $Q$, i.e. $A Q=Q \widetilde{A}$ et $B Q=Q \widetilde{B}$, alors $P:=Q U$ conjugue simultanément $A$ et $B$ à $A^{\prime}$ et $B^{\prime}$. Par ailleurs, on suppose $B \neq B^{\prime}$ (sinon le lemme est évident : choisir $P=I$ ). On distingue différents cas.

Cas 1: $A, B \in \mathcal{O}_{2}^{+}$. Notons $\alpha, \beta, \beta^{\prime} \in \mathbb{R} /(2 \pi \mathbb{Z})$ les angles respectifs des rotations $A=A^{\prime}, B$ et $B^{\prime}$. De $B \sim B^{\prime}$ et $B \neq B^{\prime}$, on déduit $\beta=-\beta^{\prime}$. De $A B \sim A B^{\prime}$ et $B \neq B^{\prime}$, on déduit $\alpha+\beta=-\left(\alpha+\beta^{\prime}\right)$, d'où $2 \alpha=0$, d'où $A= \pm I$. On peut alors choisir pour conjuguante commune $P$ toute matrice de symétrie, par exemple $S_{0}$. 
Cas 2: $A, B \in \mathcal{O}_{2}^{-}$. On a $A=S_{\alpha}, B=S_{\beta}$ et $B^{\prime}=S_{\beta^{\prime}}$. De $A B \sim A B^{\prime}$ et $B \neq B^{\prime}$, on déduit $\alpha-\beta= \pm\left(\alpha-\beta^{\prime}\right)$ et $\beta \neq \beta^{\prime}$, d'où $\alpha-\beta=-\left(\alpha-\beta^{\prime}\right)$, d'où $B A=A B^{\prime}$, et $A$ est une conjuguante commune.

Cas 3: $A \in \mathcal{O}_{2}^{+}, B \in \mathcal{O}_{2}^{-}$. On se ramène au cas 2 (dans sa forme générale $A$ éventuellement différent de $A^{\prime}$ ) en posant $\widetilde{A}=A B$ et $\widetilde{A}^{\prime}=A B^{\prime}$. Ainsi, $\widetilde{A}, B, \widetilde{A}^{\prime}$ et $B^{\prime}$ sont des symétries vérifiant $\widetilde{A} B \sim \widetilde{A}^{\prime} B^{\prime}$ puisque ces deux produits donnent $A$. D'après le cas 2 , il existe une conjuguante commune $P$, i.e. $A B P=P A B^{\prime}$ et $B P=P B^{\prime}$, d'où $P$ conjugue aussi $A$ à elle-même.

Le résultat étant symétrique en $A$ et $B$, il n'y a pas lieu de considérer le dernier cas $A \in \mathcal{O}_{2}^{-}, B \in \mathcal{O}_{2}^{+}$.

Preuve du théorème 5.2. Le sens "seulement si" est évident. Réciproquement, soit $\left(\Gamma_{1}\right.$, $\left.\ldots, \Gamma_{4}\right)$ et $\left(\Gamma_{1}^{\prime}, \ldots, \Gamma_{4}^{\prime}\right)$ deux quadruplets ayant même paramètre $\left.\left.c \in\right] 0,1\right]$ et mêmes holonomies. Soit $M\left(A,{ }^{t} C, B\right)$ et $M\left(A^{\prime},{ }^{t} C^{\prime}, B^{\prime}\right)$ des formes réduites associées. Ainsi on a $A \sim A^{\prime}, B \sim B^{\prime}, C \sim C^{\prime}, A B C \sim A^{\prime} B^{\prime} C^{\prime}, A B \sim A^{\prime} B^{\prime}, B C \sim B^{\prime} C^{\prime}$ et $C A \sim C^{\prime} A^{\prime}$. D'après le corollaire 2.7, il suffit de montrer qu'il existe une conjuguante commune $P$, i.e. telle que $A P=P A^{\prime}, B P=P B^{\prime}$ et $C P=P C^{\prime}$.

Dans le cas où l'une des trois matrices $A, B$ ou $C$ est dans le centre de $\mathcal{O}_{2}$

$$
Z\left(\mathcal{O}_{2}\right)=\{I,-I\}
$$

le lemme 5.3 permet de conclure rapidement. Par exemple, si $A= \pm I$, alors $A=A^{\prime}$ et, puisque $B \sim B^{\prime}, C \sim C^{\prime}, B C \sim B^{\prime} C^{\prime}$, d'après ce lemme il existe une conjuguante commune de $B$ à $B^{\prime}$ et de $C$ à $C^{\prime}$; cette conjuguante laissera $A$ fixe.

On suppose à présent que ni $A$ ni $B$ ni $C$ ne sont dans $Z\left(\mathcal{O}_{2}\right)$. Par une permutation adéquate des sommets $\Gamma_{j}$, on se ramène au cas où $M\left(A,{ }^{t} C, B\right)$ est de l'un des trois types donnés par la proposition 4.2.

Le lemme 5.3 permet aussi, par une réduction analogue à celle du début de la preuve du lemme, de supposer sans perte de généralité, que $A=A^{\prime}$ et $B=B^{\prime}$. En effet, d'après ce lemme, il existe $U \in \mathcal{O}_{2}$ telle que $A U=U A^{\prime}$ et $B U=U B^{\prime}$. Si on pose $\widetilde{A}=A=U A^{\prime} U^{-1}, \widetilde{B}=B=U B^{\prime} U^{-1}$ et $\widetilde{C}=U C^{\prime} U^{-1}$, alors les hypothèses du théorème sont toujours satisfaites pour $(A, B, C)$ et $(\widetilde{A}, \widetilde{B}, \widetilde{C})$. Si dans ce cas réduit il existe une même matrice $P \in \mathcal{O}_{2}$ qui conjugue simultanément $A, B$ et $C$ à $\widetilde{A}, \widetilde{B}$ et $\widetilde{C}$, alors $P U$ conjuguera simultanément $A, B$ et $C$ à $A^{\prime}, B^{\prime}$ et $C^{\prime}$.

On distingue à nouveau trois cas, suivant le type de $M\left(A,{ }^{t} C, B\right)$.

Type I : $A, B, C, C^{\prime} \in \mathcal{O}_{2}^{+}$. Si $C \neq C^{\prime}$, alors seule une symétrie peut conjuguer $C$ à $C^{\prime}$, mais cette symétrie ne peut pas laisser $A$ fixe, puisque $A$ est supposée différente de $I$ et $-I$. D'après le lemme 5.3 , ceci contredit $C \sim C^{\prime}$ et $C A \sim C^{\prime} A$. Ainsi $C=C^{\prime}$ et les formes réduites sont déjà égales.

Type II : $A, B, C, C^{\prime} \in \mathcal{O}_{2}^{-}$. On a $A=S_{\alpha}, B=S_{\beta}, C=S_{\gamma}$ et $C^{\prime}=S_{\gamma^{\prime}}$ avec $S_{\varphi}=\left(\begin{array}{cc}\cos \varphi & \sin \varphi \\ \sin \varphi & -\cos \varphi\end{array}\right)$. Les hypothèses $B C \sim B C^{\prime}$ et $C A \sim C^{\prime} A$ se traduisent par $\beta-\gamma= \pm\left(\beta-\gamma^{\prime}\right)$ et $\gamma-\alpha= \pm\left(\gamma^{\prime}-\alpha\right)$. Si $C=C^{\prime}$ il n'y a rien à faire, sinon c'est que les 
\pm sont des -, ce qui donne $2 \alpha=\gamma+\gamma^{\prime}=2 \beta$, autrement dit les axes des symétries $A$ et $B$ sont confondus ou orthogonaux. On choisit par exemple comme conjuguante commune $P=A=S_{\alpha}$ : elle fixe $A$ ainsi que $B$ et échange $C$ et $C^{\prime}$.

Type III : $A, B \in \mathcal{O}_{2}^{+}, C, C^{\prime} \in \mathcal{O}_{2}^{-}$. L'une des deux rotations qui conjugue $C$ à $C^{\prime}$ fixe $A$ et $B$, donc convient comme conjuguante commune.

Remarque. L'exemple des quadruplets de formes réduites $M\left(S_{\alpha}, S_{0}, S_{\beta}\right)$ et $M\left(S_{\alpha}, S_{0}\right.$, $\left.S_{-\beta}\right)$ montre que 6 holonomies parmi les 7 ne suffisent pas à caractériser la classe de congruence du quadruplet : les classes de $A, B, C, A B C, B C$ et $C A$ sont identiques pour les deux, mais pas celles de $A B$ si $|\alpha-\beta| \neq|\alpha+\beta|$.

Dans le cas de la dimension $d=2$, le corollaire 2.7 permet de donner un critère de congruence simple de $n$-uplets suivant leur type. Pour le type I, le résultat est le suivant.

Proposition 5.4. Soit $\left(\Gamma_{j}\right)$ et $\left(\widetilde{\Gamma}_{j}\right)$ deux $n$-uplets de plans de type I, i.e. ayant des matrices réduites $M\left(A_{i j}\right)$ et $M\left(B_{i j}\right), 2 \leq i<j \leq n$, avec $A_{i j}, B_{i j} \in \mathcal{O}_{2}^{+}$. Alors ces $n$-uplets sont congruents si et seulement si :

- ou bien $B_{i j}=A_{i j}$ pour tout couple $(i, j)$,

- ou bien $B_{i j}={ }^{t} A_{i j}$ pour tout couple $(i, j)$.

Preuve. La condition est clairement suffisante : dans le premier cas, $I$ est une conjuguante commune, dans le deuxième cas, $S_{0}$ est une conjuguante commune. Réciproquement, si $\left(\Gamma_{j}\right)$ et $\left(\widetilde{\Gamma}_{j}\right)$ sont congruents, alors il existe une conjuguante commune. Si cette conjuguante est dans $\mathcal{O}_{2}^{+}$nous sommes dans le premier cas, sinon nous sommes dans le deuxième cas.

Soit à présent $\left(\Gamma_{j}\right)$ un $n$-uplet de plans de type II ou de l'un des types mixtes. Il a donc une forme réduite $M\left(A_{i j}\right)_{2 \leq i<j \leq n}$ dont l'une des isométries $A_{i_{0} j_{0}}$ est dans $\mathcal{O}_{2}^{-}$. Quitte à conjuguer simultanément par une rotation adéquate, on peut supposer sans perte de généralité que $A_{i_{0} j_{0}}=S_{0}$. Soit $\left(\widetilde{\Gamma}_{j}\right)$ un autre $n$-uplet de plans, de forme réduite $M\left(B_{i j}\right)$. Une condition nécessaire pour que ce $n$-uplet soit congruent à $\left(\Gamma_{j}\right)$ est que $B_{i_{0} j_{0}}$ soit aussi dans $\mathcal{O}_{2}^{-}$, qu'on peut donc aussi supposer égale à $S_{0}$ sans perte de généralité. Ceci explique l'hypothèse faite dans notre prochain énoncé.

Proposition 5.5. Soit $\left(\Gamma_{j}\right)$ et $\left(\widetilde{\Gamma}_{j}\right)$ deux $n$-uplets de plans ayant des formes réduites $M\left(A_{i j}\right)$ et $M\left(B_{i j}\right), 2 \leq i<j \leq n$, avec $A_{i_{0} j_{0}}=B_{i_{0} j_{0}}=S_{0}$ pour un certain couple $\left(i_{0}, j_{0}\right)$. Si $A_{i j}=S_{\omega}$ on note $A_{i j}^{\prime}=S_{-\omega} ;$ si $A_{i j}=R_{\omega}$, on note $A_{i j}^{\prime}=R_{-\omega}$. Alors ces $n$-uplets sont congruents si et seulement si :

- ou bien $B_{i j}=A_{i j}$ pour tout couple $(i, j)$,

- ou bien $B_{i j}=A_{i j}^{\prime}$ pour tout couple $(i, j)$.

Preuve. La condition est suffisante : dans le premier cas, $I$ est une conjuguante commune, dans le deuxième cas, $S_{0}$ est une conjuguante commune. Réciproquement, si $\left(\Gamma_{j}\right)$ et $\left(\Gamma_{j}^{\prime}\right)$ sont congruents, alors il existe une conjuguante commune, qui doit fixer $S_{0}$, donc cette 
conjuguante ne peut être que $\pm I$, ce qui entraîne le premier cas, ou bien $\pm S_{0}$, qui entraîne le deuxième cas.

Théorème 5.6. 1. Deux n-uplets de type I ayant leurs sous-quadruplets correspondants congruents sont congruents.

2. Deux n-uplets de type II ayant leurs sous-quadruplets correspondants congruents sont congruents.

3. Il existe des quintuplets d'un type mixte non congruents ayant leurs sous-quadruplets correspondants congruents.

Preuve. 1. Voir proposition 5.1 et [3], proposition 1.

2. Soit $\left(\Gamma_{j}\right)$ et $\left(\widetilde{\Gamma}_{j}\right)$ deux tels $n$-uplets de type II et $M\left(A_{i j}\right), M\left(B_{i j}\right)$ des formes réduites associées, avec $2 \leq i<j \leq n$. On peut supposer sans perte de généralité que $A_{23}=$ $B_{23}=S_{0}$. On note $\alpha_{i j}$ et $\beta_{i j}$ les angles de $A_{i j}$ et $B_{i j}$. On a ainsi $A_{i j}=S_{\alpha_{i j}}$ et $B_{i j}=$ $S_{\beta_{i j}}$.

La preuve se fait en deux étapes. On montre d'abord

$$
\forall i<j \quad\left(B_{i j}=A_{i j} \text { ou } B_{i j}=A_{i j}^{\prime}\right),
$$

puis dans une deuxième étape

$$
\left(\forall i<j \quad B_{i j}=A_{i j}\right) \text { ou }\left(\forall i<j \quad B_{i j}=A_{i j}^{\prime}\right),
$$

La proposition 5.5 permet alors de conclure.

Etape 1. Par l'absurde, supposons qu'il existe $i<j$ tels que $B_{i j}$ soit différent à la fois de $A_{i j}$ et de $A_{i j}^{\prime}$, et soit $i$ le plus petit indice ayant cette propriété, pour un certain $j$. On a donc (rappelons que l'égalité des angles est sous-entendue modulo $2 \pi$ )

$$
\beta_{i j} \neq \alpha_{i j} \text { et } \beta_{i j} \neq-\alpha_{i j} .
$$

On ne peut conjuguer $A_{i j}$ à $B_{i j}$ que par $\pm R_{\alpha}$ ou $\pm S_{\beta}$ avec $\alpha=\frac{1}{2}\left(\alpha_{i j}-\beta_{i j}\right)$ et $\beta=$ $\frac{1}{2}\left(\alpha_{i j}+\beta_{i j}\right)$. Les conditions (12) entraînent $\alpha \not \equiv 0[\pi]$ et $\alpha \not \equiv \alpha_{i j}[\pi]$ dans le cas où $A_{i j}$ et $B_{i j}$ sont conjuguées par une rotation et $\beta \not \equiv 0[\pi]$ et $\beta \not \equiv \alpha_{i j}[\pi]$ dans le cas d'une symétrie.

$\mathrm{Si} i=2$ ou 3, alors d'après la proposition 5.5 les quadruplets $\left(\Gamma_{1}, \Gamma_{2}, \Gamma_{3}, \Gamma_{j}\right)$ et $\left(\widetilde{\Gamma}_{1}, \widetilde{\Gamma}_{2}, \widetilde{\Gamma}_{3}, \widetilde{\Gamma}_{j}\right)$ ne seraient pas congruents, ce qui contredit notre hypothèse.

Si $i \geq 4$, alors pour $h \in\{2,3\}$ le quadruplet $\left(\Gamma_{1}, \Gamma_{h}, \Gamma_{i}, \Gamma_{j}\right)$ a pour forme réduite $M\left(A_{h i}, A_{h j}, A_{i j}\right)$ et $\left(\widetilde{\Gamma}_{1}, \widetilde{\Gamma}_{h}, \widetilde{\Gamma}_{i}, \widetilde{\Gamma}_{j}\right)$ a pour forme réduite $M\left(B_{h i}, B_{h j}, B_{i j}\right) . \mathrm{La}$ congruence de ces quadruplets entraîne :

- ou bien $B_{h i}=S_{\alpha_{h i}-2 \alpha}$ et $B_{h j}=S_{\alpha_{h j}-2 \alpha}$ si la conjuguante est $\pm R_{\alpha}$,

- ou bien $B_{h i}=S_{2 \beta-\alpha_{h i}}$ et $B_{h j}=S_{2 \beta-\alpha_{h j}}$ si la conjuguante est $\pm S_{\beta}$.

La minimalité de $i$ implique $B_{h i}=A_{h i}$ ou $B_{h i}=A_{h i}^{\prime}$, et $B_{h j}=A_{h j}$ ou $B_{h j}=A_{h j}^{\prime}$. Puisque $\alpha \neq \equiv 0[\pi]$ et $\beta \not \equiv 0[\pi]$, cela entraîne ou bien $B_{h i}=A_{h i}^{\prime}$ et $B_{h j}=A_{h j}^{\prime}$, ou bien $B_{h i}=A_{h i}$ et $B_{h j}=A_{h j}$. Ainsi, pour chaque $h=2$ ou 3 : 
- ou bien $\alpha_{h i} \equiv \alpha[\pi]$ et $\alpha_{h j} \equiv \alpha[\pi]$,

- ou bien $\alpha_{h i} \equiv \beta[\pi]$ et $\alpha_{h j} \equiv \beta[\pi]$.

On en déduit que les quadruplets $\left(\Gamma_{2}, \Gamma_{3}, \Gamma_{i}, \Gamma_{j}\right)$ et $\left(\widetilde{\Gamma}_{2}, \widetilde{\Gamma}_{3}, \widetilde{\Gamma}_{i}, \widetilde{\Gamma}_{j}\right)$ ont des formes réduites de la forme

- $M\left(\varepsilon_{1} S_{0}, \varepsilon_{2} S_{0}, \varepsilon_{3} S_{2 \alpha-\alpha_{i j}}\right)$ et $M\left(\varepsilon_{1} S_{0}, \varepsilon_{2} S_{0}, \varepsilon_{3} S_{-2 \alpha-\beta_{i j}}\right)$ avec $\varepsilon_{k}= \pm 1$ dans le cas $\pm R_{\alpha}$,

- $M\left(\varepsilon_{1} S_{0}, \varepsilon_{2} S_{0}, \varepsilon_{3} S_{2 \beta-\alpha_{i j}}\right)$ et $M\left(\varepsilon_{1} S_{0}, \varepsilon_{2} S_{0}, \varepsilon_{3} S_{2 \beta-\beta_{i j}}\right)$ avec $\varepsilon_{k}= \pm 1$ dans le cas $\pm S_{\beta}$,

donc ces quadruplets ne seraient pas congruents.

Etape 2. Supposons qu'il existe $i<j$ avec $\beta_{i j} \neq \alpha_{i j}$ et $k<l$ avec $\beta_{k l} \neq-\alpha_{k l}$. D'après l'étape 1 , on a donc $\beta_{i j}=-\alpha_{i j}$ et $\beta_{k l}=\alpha_{k l}$. En particulier ni $\alpha_{i j}$ ni $\alpha_{k l}$ ne sont congrus à 0 modulo $\pi$.

- Si $i=k$, alors nécessairement $j \neq l$, et les quadruplets $\left(\Gamma_{1}, \Gamma_{i}, \Gamma_{j}, \Gamma_{l}\right)$ et $\left(\widetilde{\Gamma}_{1}, \widetilde{\Gamma}_{i}, \widetilde{\Gamma}_{j}\right.$, $\left.\widetilde{\Gamma}_{l}\right)$, de formes réduites $M\left(A_{i j}, A_{k l}, A_{j l}\right)$ et $M\left(B_{i j}, B_{k l}, B_{j l}\right)$, ne sont pas congruents, ce qui est impossible.

- De même, si $j=l$, alors $i \neq k$, et les quadruplets $\left(\Gamma_{1}, \Gamma_{i}, \Gamma_{j}, \Gamma_{k}\right)$ et $\left(\widetilde{\Gamma}_{1}, \widetilde{\Gamma}_{i}, \widetilde{\Gamma}_{j}, \widetilde{\Gamma}_{k}\right)$ ne sont pas congruents, ce qui est impossible.

- Si $i \neq k$ et $j \neq l$, alors compte tenu de $i<j$ et $k<l$, soit on a $i=l$ et $j \neq k$, soit $i \neq l$ et $j=k$, soit les quatre indices sont deux à deux distincts.

Si $i=l$, la contradiction vient du fait que $\left(\Gamma_{1}, \Gamma_{i}, \Gamma_{j}, \Gamma_{k}\right)$ et $\left(\widetilde{\Gamma}_{1}, \widetilde{\Gamma}_{i}, \widetilde{\Gamma}_{j}, \widetilde{\Gamma}_{k}\right)$, de formes réduites $M\left(A_{i j}, A_{l k}, A_{j k}\right)$ et $M\left(B_{i j}, B_{l k}, B_{j k}\right)$, ne sont pas congruents.

Si $j=k$, la contradiction vient du fait que $\left(\Gamma_{1}, \Gamma_{i}, \Gamma_{j}, \Gamma_{l}\right)$ et $\left(\widetilde{\Gamma}_{1}, \widetilde{\Gamma}_{i}, \widetilde{\Gamma}_{j}, \widetilde{\Gamma}_{l}\right)$ ne sont pas congruents.

Il reste le cas où les quatre indices sont deux à deux distincts. Dans ce cas, la congruence des quadruplets $\left(\Gamma_{1}, \Gamma_{i}, \Gamma_{j}, \Gamma_{k}\right)$ et $\left(\widetilde{\Gamma}_{1}, \widetilde{\Gamma}_{i}, \widetilde{\Gamma}_{j}, \widetilde{\Gamma}_{k}\right)$ et le fait que $\alpha_{i j} \not \equiv 0[\pi] \mathrm{im}$ pliquent $B_{i k}=A_{i k}^{\prime}$ et $B_{j k}=A_{j k}^{\prime}$. De même, la congruence des quadruplets $\left(\Gamma_{1}, \Gamma_{i}, \Gamma_{j}\right.$, $\left.\Gamma_{l}\right)$ et $\left(\widetilde{\Gamma}_{1}, \widetilde{\Gamma}_{i}, \widetilde{\Gamma}_{j}, \widetilde{\Gamma}_{l}\right)$ et le fait que $\alpha_{i j} \not \equiv 0[\pi]$ impliquent $B_{i l}=A_{i l}^{\prime}$ et $B_{j l}=A_{j l}^{\prime}$. Ces faits et la congruence des quadruplets $\left(\Gamma_{1}, \Gamma_{j}, \Gamma_{k}, \Gamma_{l}\right)$ et $\left(\widetilde{\Gamma}_{1}, \widetilde{\Gamma}_{j}, \widetilde{\Gamma}_{k}, \widetilde{\Gamma}_{l}\right)$ et celle des quadruplets $\left(\Gamma_{1}, \Gamma_{i}, \Gamma_{k}, \Gamma_{l}\right)$ et $\left(\widetilde{\Gamma}_{1}, \widetilde{\Gamma}_{i}, \widetilde{\Gamma}_{k}, \widetilde{\Gamma}_{l}\right)$ entraînent alors $\alpha_{j k}=\beta_{j k} \equiv 0[\pi], \alpha_{j l}=$ $\beta_{j l} \equiv 0[\pi], \alpha_{i k}=\beta_{i k} \equiv 0[\pi]$ et $\alpha_{i l}=\beta_{i l} \equiv 0[\pi]$.

Finalement, les quadruplets $\left(\Gamma_{i}, \Gamma_{j}, \Gamma_{k}, \Gamma_{l}\right)$ et $\left(\widetilde{\Gamma}_{i}, \widetilde{\Gamma}_{j}, \widetilde{\Gamma}_{k}, \widetilde{\Gamma}_{l}\right)$, de formes réduites $M\left(\varepsilon_{1} A_{i j}, \varepsilon_{2} A_{j k}, A_{k l}\right)$ et $M\left(\varepsilon_{1} B_{i j}, \varepsilon_{2} B_{j k}, B_{k l}\right)$ avec $\varepsilon_{1}=\varepsilon_{2}= \pm 1$, ne sont pas congruents, ce qui est impossible.

3. Soit $\left(\Gamma_{i}\right)_{i=1, \ldots, 5}$ le quintuplet de forme réduite

$$
M(A, B, C, D, E, F)=\left(\begin{array}{ccccc}
0 & I & I & I & I \\
I & 0 & A & B & C \\
I & { }^{t} A & 0 & D & E \\
I & { }^{t} B & { }^{t} D & 0 & F \\
I & { }^{t} C & { }^{t} E & { }^{t} F & 0
\end{array}\right)
$$


où les blocs $A, \ldots, F$ seront précisés plus loin. Notons $Q_{j}$ le sous-quadruplet obtenu en supprimant $\Gamma_{j}$. Ainsi, des formes réduites associées aux cinq sous-quadruplets sont $M(A, B, D)$ pour $Q_{5}, M(A, C, E)$ pour $Q_{4}, M(B, C, F)$ pour $Q_{3}, M(D, E, F)$ pour $Q_{2}$ et $M\left(A D^{t} B, A E^{t} C, B F^{t} C\right)$ pour $Q_{1}$. Notons de même avec des primes les éléments d'un autre quintuplet.

Choisissons $A=B=C=R_{\pi / 2}, D=F=S_{0}$ et $E=S_{\pi / 2}$ pour le premier quintuplet, et $A^{\prime}=B^{\prime}=C^{\prime}={ }^{t} A, D^{\prime}=D, E^{\prime}=E, F^{\prime}=F$ pour le deuxième. On obtient ainsi $A D^{t} B=-D=A^{\prime} D^{t} B^{\prime}, A E^{t} C=-E=A^{\prime} E^{t} C^{\prime}$ et $B F^{t} C=-F=B^{\prime} F^{t} C^{\prime}$. On vérifie que les matrices $P_{j}$ suivantes sont des conjuguantes communes entre $Q_{j}$ et $Q_{j}^{\prime}: P_{5}=S_{0}, P_{4}=S_{\pi / 2}, P_{3}=S_{0}, P_{2}=I$ et $P_{1}=S_{0}$. Les sous-quadruplets correspondants sont donc congruents. Par contre une conjuguante commune entre les quintuplets ne peut être qu'une symétrie $S_{\omega}$ pour conjuguer $A$ à $A^{\prime}$, et $\omega$ doit vérifier $\omega=0$ ou $\pi$ pour fixer $D$ et $F$, et en même temps $\omega=\frac{\pi}{2}$ ou $-\frac{\pi}{2}$ pour fixer $E$, ce qui est impossible. Il se trouve que les spectres des matrices de Seidel associées coïncident. Ils sont d'ailleurs symétriques par rapport à $0:$ on trouve pour polynôme caractéristique des deux matrices $\left(x^{2}-1\right)\left(x^{8}-19 x^{6}+91 x^{4}-109 x^{2}+4\right)$.

\section{Les $n$-uplets réguliers de plans}

Nous dressons dans cette partie la liste exhaustive des quadruplets réguliers de $G(2,8)$, puis nous verrons lesquels peuvent être complétés en $n$-uplets réguliers de cardinal $n \geq 5$.

Théorème 6.1. Les quadruplets réguliers de $G(2,8)$ se répartissent en cinq familles : trois de type I et deux de type II.

Les trois familles de type I ont des formes réduites :

- $\quad M(I, I, I)$ avec $c \in] 0,1]$, d'ordre de platitude nul pour $c<1$ et 6 pour $c=1$,

- $M(-I,-I,-I)$ avec $\left.c \in] 0, \frac{1}{3}\right]$, d'ordre de platitude nul pour $c<\frac{1}{3}$ et 2 pour $c=\frac{1}{3}$,

- $\quad M\left(R_{\pi / 2}, R_{-\pi / 2}, R_{\pi / 2}\right)$ avec $\left.\left.c \in\right] 0, \frac{1}{\sqrt{3}}\right]$, d'ordre de platitude nul pour $c<\frac{1}{\sqrt{3}}$ et 4 pour $c=\frac{1}{\sqrt{3}}$.

Les deux familles de type II ont des formes réduites:

- $M\left(S_{0}, S_{0}, S_{0}\right)$ avec $\left.\left.c \in\right] 0, \frac{1}{3}\right]$, d'ordre de platitude nul pour $c<\frac{1}{3}$ et 1 pour $c=\frac{1}{3}$,

- $M\left(S_{-2 \pi / 3}, S_{0}, S_{2 \pi / 3}\right)$ avec $\left.\left.c \in\right] 0, \frac{1}{2}\right]$, d'ordre de platitude nul pour $c<\frac{1}{2}$ et 3 pour $c=\frac{1}{2}$.

Preuve. Soit $\left(\Gamma_{1}, \Gamma_{2}, \Gamma_{3}, \Gamma_{4}\right)$ un quadruplet de $G(2,8)$ et $M\left(A,{ }^{t} C, B\right)$ une matrice de Seidel associée. D'après le théorème $5.2,\left(\Gamma_{1}, \Gamma_{2}, \Gamma_{3}, \Gamma_{4}\right)$ est régulier si et seulement si

$$
A \sim B \sim C \sim A B C \text { et } A B \sim B C \sim C A .
$$

Nous distinguons deux cas, suivant le type. 
Type I : la matrice A est dans $\mathcal{O}_{2}^{+}$. Dans ce cas $B$ et $C$ sont aussi des matrices de rotation. On a $A=R_{\alpha}, B=R_{\beta}$ et $C=R_{\gamma}$ avec $R_{\varphi}=\left(\begin{array}{cc}\cos \varphi & -\sin \varphi \\ \sin \varphi & \cos \varphi\end{array}\right)$. Les conditions (13) donnent $\alpha= \pm \beta= \pm \gamma= \pm(\alpha+\beta+\gamma)$ et $\alpha+\beta= \pm(\beta+\gamma)= \pm(\gamma+\alpha)$ (rappelons que les égalités sont modulo $2 \pi$ ). Si $\alpha=-\beta$, alors $0=\alpha+\beta=\beta+\gamma=\gamma+\alpha$, d'où $\alpha=\beta$, donc dans tous les cas, (13) implique que $\alpha=\beta$ et de même $\alpha=\gamma$. La condition $\alpha= \pm(\alpha+\beta+\gamma)$ entraîne alors $4 \alpha=0$, d'où $\alpha=\beta=\gamma$ prend l'une des valeurs $0, \frac{\pi}{2}, \pi$ ou $\frac{3 \pi}{2}$. Les cas $\alpha=0$ et $\alpha=\pi$ correspondent aux deux premières familles. Les deux cas $\alpha=\frac{\pi}{2}$ et $\alpha=\frac{3 \pi}{2}$ donnent des quadruplets congruents, dont une forme réduite est $M\left(R,{ }^{t} R, R\right)$ avec $R=R_{\pi / 2}$.

Type II : la matrice $A$ est dans $\mathcal{O}_{2}^{-}$. Dans ce cas $B$ et $C$ sont aussi des matrices de symétrie : $A=S_{\alpha}, B=S_{\beta}$ et $C=S_{\gamma}$ avec $S_{\varphi}=\left(\begin{array}{cc}\cos \varphi & \sin \varphi \\ \sin \varphi & -\cos \varphi\end{array}\right)$. Les premières conditions de (13) $A \sim B \sim C \sim A B C$ sont toujours réalisées. On obtient $A B=R_{\alpha-\beta}$, donc les deuxièmes conditions de (13) donnent

$$
\alpha-\beta= \pm(\beta-\gamma)= \pm(\gamma-\alpha) .
$$

Si $\alpha=\beta$ alors (14) donne aussi $\alpha=\gamma$, d'où $M\left(A,{ }^{t} C, B\right)=M\left(S_{\alpha}, S_{\alpha}, S_{\alpha}\right)$. En conjuguant simultanément par $R_{\alpha / 2}$ (remarquer que $R_{-\alpha / 2} S_{\alpha} R_{\alpha / 2}=S_{0}$ ), on en déduit qu'une forme réduite du quadruplet est $M\left(S_{0}, S_{0}, S_{0}\right)$, ce qui donne la première famille de type II.

Si $\alpha \neq \beta$, alors on a aussi $\alpha \neq \gamma \neq \beta$, donc (14) donne $\alpha-\beta=\beta-\gamma=\gamma-\alpha$, d'où on déduit successivement $2 \beta=\alpha+\gamma$ et $2 \gamma=\alpha+\beta$, d'où $4 \beta=2 \alpha+(\alpha+\beta)$, d'où $3 \alpha=3 \beta$ et de même $3 \alpha=3 \gamma$. Puisque $\alpha \neq \beta \neq \gamma \neq \alpha$, on en déduit $\beta=\alpha+\varepsilon \frac{2 \pi}{3}$ et $\gamma=\alpha-\varepsilon \frac{2 \pi}{3}$ avec $\varepsilon= \pm 1$. En conjuguant par la rotation d'angle $\frac{\gamma}{2}$ et éventuellement une symétrie, on en déduit qu'une forme réduite du quadruplet est $M\left(S_{-2 \pi / 3}, S_{0}, S_{2 \pi / 3}\right)$, d'où la dernière famille.

Les conditions d'existence et les ordres de platitude se calculent directement à partir des spectres des formes réduites, en utilisant l'item 3 du théorème 2.3. On obtient

$$
\begin{gathered}
\operatorname{Spec} M(I, I, I)=(\underbrace{-1, \ldots,-1}_{6}, 3,3), \\
\operatorname{Spec} M(-I,-I,-I)=(-3,-3, \underbrace{1, \ldots, 1}_{6}), \\
\operatorname{Spec} M\left(R_{\pi / 2}, R_{-\pi / 2}, R_{\pi / 2}\right)=(\underbrace{-\sqrt{3}, \ldots,-\sqrt{3}}_{4}, \underbrace{\sqrt{3}, \ldots, \sqrt{3}}_{4}), \\
\operatorname{Spec} M\left(S_{0}, S_{0}, S_{0}\right)=(-3,-1,-1,-1,1,1,1,3)
\end{gathered}
$$

et

$$
\operatorname{Spec} M\left(S_{-2 \pi / 3}, S_{0}, S_{2 \pi / 3}\right)=(-2,-2,-2,0,0,2,2,2) \text {. }
$$


Nous terminons cette section par la description complète des $n$-uplets réguliers de $G(2,2 n)$.

Théorème 6.2. La liste des $n$-uplets réguliers de $G(2,2 n)$ est la suivante.

- Tous les triplets.

- Pour tout $n \geq 4$, les $n$-uplets ayant pour matrices de Seidel associées les matrices $M_{A}=J_{n} \otimes A$ décrites dans (9), avec $A=I,-I$ ou $S_{0}$.

Pour $A=I$, le $n$-uplet existe pour tout $c \leq 1$; son ordre de platitude est 0 pour $c<1,2 n-2$ pour le $n$-uplet trivial où $c=1$.

Pour $A=-I$ ou $S_{0}$, le $n$-uplet existe si et seulement si $c \leq \frac{1}{n-1}$. Si $c<\frac{1}{n-1}$, alors son ordre de platitude est 0 ; si $c=\frac{1}{n-1}$, alors cet ordre est 1 lorsque $A=S_{0}$ et 2 lorsque $A=-I$.

- Les quadruplets ayant pour matrice de Seidel associée $M\left(R_{\pi / 2}, R_{-\pi / 2}, R_{\pi / 2}\right)$, avec $\left.c \in] 0, \frac{1}{\sqrt{3}}\right]$, d'ordre de platitude nul pour $c<\frac{1}{\sqrt{3}}$ et 4 pour $c=\frac{1}{\sqrt{3}}$.

- Les quintuplets ayant pour matrice de Seidel associée $M\left(S_{-2 \pi / 3}, S_{0}, S_{2 \pi / 3}, S_{2 \pi / 3}\right.$, $\left.S_{0}, S_{-2 \pi / 3}\right)$, ainsi que leurs sous-quadruplets, avec $\left.\left.c \in\right] 0, \frac{1}{2}\right]$. Si $c<\frac{1}{2}$, alors l'ordre de platitude est nul; si $c=\frac{1}{2}$, alors cet ordre est 3 pour les quadruplets et 5 pour le quintuplet.

Preuve. Puisqu'un sous-quadruplet d'un $n$-uplet régulier doit être lui aussi régulier, il suffit d'examiner quels sont les quadruplets de la liste donnée dans le théorème 6.1 qui peuvent s'étendre.

Soit $\left(\Gamma_{j}\right)$ un $n$-uplet régulier de plans et soit $M=M\left(A_{23}, \ldots A_{n-1 n}\right)$ une forme réduite associée. Puisque les holonomies le long des 3-cycles doivent coïncider, toutes les matrices $A_{i j}, 2 \leq i<j \leq n$ sont conjuguées. En particulier, si l'une de ces matrices est dans le centre de $\mathcal{O}_{2}$ constitué de $I$ et $-I$, alors toutes sont égales. En d'autres termes les seuls $n$-uplets réguliers pouvant compléter les quadruplets des deux familles listées au début du théorème 6.1 , de formes réduites $M(I, I, I)$ et $M(-I,-I,-I)$, sont ceux ayant une matrice de Seidel de la forme $M_{A}$ décrite dans (9), avec $A=I$ ou $-I$. On a déjà vu dans la proposition 2.12 que ces $n$-uplets sont bien réguliers pour tout $n \geq 1$. Il en est de même des $n$-uplets de matrice de Seidel $M_{S_{0}}$ qui seront considérés plus bas.

Les conditions d'existence et les ordres de platitude s'obtiennent à l'aide de l'item 3 du théorème 2.3 et des spectres des matrices $M_{A}=J_{n} \otimes A$ calculés au début de la partie 2.5. On a

$$
\begin{gathered}
\operatorname{Spec} M_{I}=(\underbrace{-1, \ldots,-1}_{2 n-2}, n-1, n-1), \\
\operatorname{Spec} M_{-I}=(-n+1,-n+1, \underbrace{1, \ldots, 1}_{2 n-2})
\end{gathered}
$$

et

$$
\operatorname{Spec} M_{S_{0}}=(\underbrace{-n+1, \ldots,-n+1}_{n-1}, \underbrace{-1, \ldots,-1}_{n-1}, \underbrace{1, \ldots, 1}_{n-1}, \underbrace{n-1, \ldots, n-1}_{n-1}) .
$$


- Examinons à présent la troisième famille de type I. Notons $R$ pour $R_{\pi / 2}$. Le quadruplet de forme réduite $m=M\left(R,{ }^{t} R, R\right)$ ne peut s'étendre en un quintuplet de forme réduite

$$
M:=M\left(R,{ }^{t} R, A, R, B, C\right)=\left(\begin{array}{ccccc}
0 & I & I & I & I \\
I & 0 & R & { }^{t} R & A \\
I & { }^{t} R & 0 & R & B \\
I & R & { }^{t} R & 0 & C \\
I & { }^{t} A & { }^{t} B & { }^{t} C & 0
\end{array}\right)
$$

qu'avec $A, B, C \in\left\{{ }^{t} R, R\right\}$. Le sous-quadruplet $\left(\Gamma_{1}, \Gamma_{3}, \Gamma_{4}, \Gamma_{5}\right)$ a pour forme réduite $M(R, B, C)$ (obtenue en supprimant la deuxième ligne et la deuxième colonne de $M$ ), qui ne peut être équivalente à $m$ que si $B={ }^{t} R$ et $C=R$; mais alors le sous-quadruplet $\left(\Gamma_{1}, \Gamma_{2}, \Gamma_{4}, \Gamma_{5}\right)$, qui a pour forme réduite $M\left({ }^{t} R, A, C\right)$, n'est pas congruent à $\left(\Gamma_{1}, \Gamma_{2}, \Gamma_{3}\right.$, $\left.\Gamma_{4}\right)$. Ainsi ce quadruplet ne peut pas s'étendre.

- Concernant la première famille de type II, le quadruplet de forme réduite $M\left(S_{0}, S_{0}\right.$, $\left.S_{0}\right)$ ne peut s'étendre en un quintuplet de forme réduite $M\left(S_{0}, S_{0}, A, S_{0}, B, C\right)$ qu'avec $A, B, C$ dans $\mathcal{O}_{2}^{-}$. Les 4-holonomies sont alors égales à la classe de $S_{0} S_{0}=I$, donc $A=B=C=S_{0}$. De même, on vérifie qu'un $(n-1)$-uplet admettant une matrice de Seidel associée de la forme $M_{S_{0}, n-1}=J_{n-1} \otimes S_{0}$, i.e. d'ordre $2(n-1)$ dont les blocs non nuls sont tous égaux à $S_{0}$, comme décrit dans (9) et (11), ne peut s'étendre qu'en un $n$ uplet ayant une matrice de Seidel associée de la forme $M_{S_{0}, n}=J_{n} \otimes S_{0}$. Par récurrence, il s'ensuit que seuls ces $n$-uplets peuvent étendre ce quadruplet.

- Concernant la dernière famille, notons $S_{+}=S_{2 \pi / 3}$ et $S_{-}=S_{-2 \pi / 3}$. Concernant le quadruplet de forme réduite $M\left(S_{-}, S_{0}, S_{+}\right)$, il ne peut s'étendre en un quintuplet de forme réduite

$$
M\left(S_{-}, S_{0}, A, S_{+}, B, C\right)=\left(\begin{array}{ccccc}
0 & I & I & I & I \\
I & 0 & S_{-} & S_{0} & A \\
I & S_{-} & 0 & S_{+} & B \\
I & S_{0} & S_{+} & 0 & C \\
I & { }^{t} A & { }^{t} B & { }^{t} C & 0
\end{array}\right)
$$

qu'avec $A, B, C \in \mathcal{O}_{2}^{-}$. Les 4-holonomies sont alors égales à la classe de $S_{0} S_{+}=$ $R_{-2 \pi / 3}$.

Puisque le sous-quadruplet $\left(\Gamma_{1}, \Gamma_{3}, \Gamma_{4}, \Gamma_{5}\right)$ a pour forme réduite $M\left(S_{+}, B, C\right)$, c'est que $B$ ou $C$ est $S_{0}$ et l'autre est $S_{-}$.

Puisque le sous-quadruplet $\left(\Gamma_{1}, \Gamma_{2}, \Gamma_{4}, \Gamma_{5}\right)$ a pour forme réduite $M\left(S_{0}, A, C\right)$, c'est que $A$ ou $C$ est $S_{-}$et l'autre est $S_{+}$. Par conséquent $C=S_{-}, B=S_{0}$ et $A=S_{+}$. Autrement dit, seul un quintuplet (à congruence près) peut étendre ce quadruplet. Ce quintuplet a déjà été étudié dans [7]. En particulier il est démontré que son groupe de symétrie est bien $\mathcal{S}_{5}$. Par ailleurs, il est aussi démontré dans [7] que ce quintuplet réalise le nombre maximum de plans distincts dans $\mathbb{R}^{5}$ pour la valeur $c=\frac{1}{2}$. Ainsi l'ordre de platitude de ce quintuplet est bien 5 . On peut aussi vérifier cet ordre sur le spectre de sa forme réduite. On trouve $\operatorname{Spec} M\left(S_{-}, S_{0}, S_{+}, S_{+}, S_{0}, S_{-}\right)=(\underbrace{-2, \ldots,-2}_{5}, \underbrace{2, \ldots, 2}_{5})$. 
Il nous reste à vérifier que le quintuplet ne peut pas s'étendre en un sextuplet. Si c'était le cas, ce sextuplet aurait une forme réduite égale à

$$
M\left(S_{-}, S_{0}, S_{+}, D, S_{+}, S_{0}, E, S_{-}, F, G\right)=\left(\begin{array}{cccccc}
0 & I & I & I & I & I \\
I & 0 & S_{-} & S_{0} & S_{+} & D \\
I & S_{-} & 0 & S_{+} & S_{0} & E \\
I & S_{0} & S_{+} & 0 & S_{-} & F \\
I & S_{+} & S_{0} & S_{-} & 0 & G \\
I & D & E & F & G & 0
\end{array}\right)
$$

avec $D, E, F, G \in\left\{S_{-}, S_{0}, S_{+}\right\}$. Une matrice de Seidel du sous-quintuplet ne contenant pas $\Gamma_{5}$, obtenue en supprimant la cinquième ligne et la cinquième colonne, devrait être équivalente à $M\left(S_{-}, S_{0}, S_{+}, S_{+}, S_{0}, S_{-}\right)$, ce qui impliquerait que $D=S_{+}$, $E=S_{0}$ et $F=S_{-}$. Une matrice de Seidel du sous-quintuplet ne contenant pas $\Gamma_{4}$, obtenue en supprimant la quatrième ligne et la quatrième colonne, devrait être équivalente à $M\left(S_{-}, S_{0}, S_{+}, S_{+}, S_{0}, S_{-}\right)$, ce qui impliquerait que $D=S_{0}, E=S_{+}$et $G=S_{-}$, or ceci est impossible.

\section{$7 \quad$ Les quadruplets de plans dans $\mathbb{R}^{6}$}

La liste complète des quadruplets de plans dans $\mathbb{R}^{N}$ avec $N \leq 5$ est déjà connue [7]. Hormis le trivial, il y a, à congruence près :

- un seul quadruplet de type I, qui est d'ordre de platitude 4, obtenu avec le paramètre $c=\frac{1}{\sqrt{3}}$ et la matrice de Seidel $M\left(R_{\pi / 2}, R_{-\pi / 2}, R_{\pi / 2}\right)$. Ce quadruplet correspond à l'unique quadruplet équilatère de $\mathbb{C} P^{1}$, c.f.[8];

- un seul quadruplet de type II, d'ordre de platitude 3, qui est sous-quadruplet du quintuplet de $\mathbb{R}^{5}$ décrit dans [7].

Le but de cette partie est de déterminer tous les quadruplets de $G(2,6)$, donc d'ordre de platitude au moins 2 .

On considère donc $\left(\Gamma_{1}, \Gamma_{2}, \Gamma_{3}, \Gamma_{4}\right)$ un quadruplet de $G(2,8)$ de paramètre $\left.c \in\right] 0,1[$ et de forme réduite $M\left(A,{ }^{t} C, B\right)$ de l'un des trois types décrits dans la proposition 4.2, on note $\mu_{0}(A, B, C)$ la plus petite valeur propre de $M\left(A,{ }^{t} C, B\right)$, et il s'agit de déterminer la multiplicité de celle-ci.

L'étude des quadruplets de type I a déjà été faite en grande partie dans $[7,8]$ : comme le montre la proposition 5.1, ces quadruplets correspondent à des quadruplets équilatères de $\mathbb{C} P^{3}$. Le résultat qui suit a donc été déjà démontré en grande partie; cependant, pour la complétude du présent article, nous donnons la preuve.

Théorème 7.1. Soit $\left(\Gamma_{1}, \Gamma_{2}, \Gamma_{3}, \Gamma_{4}\right)$ un quadruplet de $G(2,8)$ de type I et $M\left(A,{ }^{t} C, B\right)$ une forme réduite associée (donc $A, B, C \in \mathcal{O}_{2}^{+}$).

L'ordre de platitude de $\left(\Gamma_{j}\right)$ est toujours pair.

Dans le cas trivial où $A=B=C=I$ et $c=1$, alors cet ordre est 6 . 
Dans le cas où le quadruplet admet pour matrice de Seidel $M\left(R_{\pi / 2}, R_{-\pi / 2}, R_{\pi / 2}\right)$ et a pour paramètre $c=\frac{1}{\sqrt{3}}$, alors cet ordre est 4 .

Dans tous les autres cas, si $c=-\frac{1}{\mu_{0}(A, B, C)}$, alors cet ordre est 2 .

Dans tous les cas, si $c<-\frac{1}{\mu_{0}(A, B, C)}$ alors cet ordre est nul.

Preuve. Soit $\alpha, \beta$ et $\gamma$ les angles respectifs de $A, B$ et $C$. Comme nous l'avons vu juste avant la proposition 5.1 , le polynôme caractéristique de $M\left(A,{ }^{t} C, B\right)$ est le carré de celui de la matrice

$$
m(\alpha,-\gamma, \beta):=\left(\begin{array}{cccc}
0 & 1 & 1 & 1 \\
1 & 0 & e^{i \alpha} & e^{-i \gamma} \\
1 & e^{-i \alpha} & 0 & e^{i \beta} \\
1 & e^{i \gamma} & e^{-i \beta} & 0
\end{array}\right) .
$$

Par conséquent la multiplicité de toute valeur propre, en particulier $\mu_{0}(A, B, C)$, est paire.

Si $M\left(A,{ }^{t} C, B\right)$ a une valeur propre $\lambda$ de multiplicité strictement supérieure à 2 , alors $\lambda$ est une valeur propre de $m(\alpha,-\gamma, \beta)$ de multiplicité au moins 2 , donc tous les mineurs d'ordre 3 de la matrice $\lambda I-m(\alpha,-\gamma, \beta)$ sont nuls. En désignant par $M_{i j}$ le mineur d'ordre 3 obtenu en ôtant la $i$-ième ligne et la $j$-ième colonne, on obtient $M_{11}=\lambda^{3}-$ $3 \lambda-2 \cos (\alpha+\beta+\gamma), M_{22}=\lambda^{3}-3 \lambda-2 \cos \beta, M_{33}=\lambda^{3}-3 \lambda-2 \cos \gamma$ et $M_{44}=$ $\lambda^{3}-3 \lambda-2 \cos \alpha$. Une condition nécessaire pour que l'ordre de platitude du triplet soit strictement supérieur à 2 est donc

$$
\cos \alpha=\cos \beta=\cos \gamma=\cos (\alpha+\beta+\gamma) .
$$

Ainsi quatre cas peuvent se produire (éventuellement redondants si $\alpha=0 \bmod \pi$ ) :

(i) $\alpha=\beta=\gamma= \pm \frac{\pi}{2}$, (ii) $\alpha=\beta=-\gamma$, (iii) $\alpha=-\beta=-\gamma$ et (iv) $\alpha=-\beta=\gamma$.

Dans le cas (i), on passe de $\alpha=\beta=\gamma=-\frac{\pi}{2}$ à $\alpha=\beta=\gamma=\frac{\pi}{2}$ en conjuguant simultanément les blocs lignes et colonnes par une symétrie. Nous retrouvons le quadruplet régulier, d'ordre de platitude 4 si $c=\frac{1}{\sqrt{3}}$.

Concernant les trois autres cas, d'après la table 1 , une permutation des sommets $\Gamma_{1}$ et $\Gamma_{2}$ conduit à une forme réduite $M\left({ }^{t} A, C, A B C\right)$. Ainsi, par la transposition (12) on passe de $M(A, A, A)$ (correspondant au cas (ii)) à $M\left({ }^{t} A,{ }^{t} A, A\right)$ (cas (iii)). De même la transposition (23) donne $M\left({ }^{t} A, A, A\right)$ (cas (iv)). Autrement dit, les deux cas (iii) et (iv) se ramènent au cas (ii) $M(A, A, A)$ par une permutation des sommets. Noter que ces trois cas correspondent à des quadruplets non réguliers, contrairement au cas (i).

Dans le cas (ii), le polynôme caractéristique de la matrice $m(\alpha, \alpha, \alpha)$ a pour racines $1 \pm \sqrt{2+2 \cos \alpha}$ et $-1 \pm \sqrt{2-2 \cos \alpha}$, donc la plus petite n'est au moins double que si $\cos \alpha=1$, ce qui correspond au quadruplet trivial.

Par ordre croissant de difficulté, viennent ensuite les quadruplets de type II, où toutes les 3-holonomies sont négatives.

Théorème 7.2. Si un quadruplet de $G(2,8)$ de type II a un ordre de platitude supérieur ou égal à 2, alors, quitte à permuter ses sommets, il admet une forme réduite $M\left(S_{\alpha}, S_{0}\right.$, $S_{-\alpha)}$, avec $\alpha \in\left[\frac{2 \pi}{3}, \pi\right]$. 
Réciproquement, pour tout $\left.\alpha \in] \frac{2 \pi}{3}, \pi\right]$, le quadruplet de paramètre $(3-2 \cos \alpha)^{-1 / 2}$ et de forme réduite $M\left(S_{\alpha}, S_{0}, S_{-\alpha}\right)$ a pour ordre de platitude 2. Pour $\alpha=\frac{2 \pi}{3}$, le quadruplet de paramètre $c=\frac{1}{2}$ et de forme réduite $M\left(S_{\alpha}, S_{0}, S_{-\alpha}\right)$ a pour ordre de platitude 3 .

Preuve. Soit $\left(\Gamma_{1}, \ldots, \Gamma_{4}\right)$ un tel quadruplet et soit $M:=M\left(S_{\alpha}, S_{\gamma}, S_{\beta}\right)$ une forme réduite. Quitte à conjuguer simultanément $S_{\alpha}, S_{\beta}$ et $S_{\gamma}$ par $R_{\gamma / 2}$, on peut supposer $\gamma=0$. Puisque $\left(\Gamma_{j}\right)$ a un ordre de platitude supérieur ou égal à 2 , c'est que $\mu_{0}(A, B, C)$ est de multiplicité au moins 2 , donc le polynôme caractéristique de $M$, noté $P_{M}$, a nécessairement une racine double. Le plus simple est d'utiliser une paramétrisation rationnelle en posant $u=\tan \frac{\alpha}{2}$ et $v=\tan \frac{\beta}{2}$. Ainsi la matrice $M$ a la forme

$$
\widetilde{M}(u, v)=\left(\begin{array}{cccc}
0 & I & I & I \\
I & 0 & S_{\alpha} & S_{0} \\
I & S_{\alpha} & 0 & S_{\beta} \\
I & S_{0} & S_{\beta} & 0
\end{array}\right)
$$

avec

$$
\begin{gathered}
S_{0}=\left(\begin{array}{cc}
1 & 0 \\
0 & -1
\end{array}\right), \quad S_{\alpha}=\left(\begin{array}{cc}
a & s_{a} \\
s_{a} & -a
\end{array}\right), \quad S_{\beta}=\left(\begin{array}{cc}
b & s_{b} \\
s_{b} & -b
\end{array}\right), \\
\cos \alpha=a=\frac{1-u^{2}}{1+u^{2}}, \quad \sin \alpha=s_{a}=\frac{2 u}{1+u^{2}}, \\
\cos \beta=b=\frac{1-v^{2}}{1+v^{2}}, \quad \sin \beta=s_{b}=\frac{2 v}{1+v^{2}} .
\end{gathered}
$$

Il revient au même de donner des conditions sur $u$ et $v$ pour que le polynôme caractéristique $P_{A}$ de la matrice $A(u, v):=\left(1+u^{2}\right)\left(1+v^{2}\right) \widetilde{M}(u, v)$ ait une racine double, l'avantage étant que les coefficients de $A$ sont polynomiaux en $u$ et $v$. Pour cela on détermine le discriminant de $P_{A}$, noté $\Delta(u, v)$. On trouve :

$\Delta(u, v)=8^{8}\left(1+u^{2}\right)^{18}\left(1+v^{2}\right)^{18} u^{2} v^{2}(u+v)^{2}(u-v)^{2}\left(2 u-v+u^{2} v\right)^{2}\left(2 v-u+u v^{2}\right)^{2}$.

Les conditions sur $u$ et $v$ pour annuler $\Delta(u, v)$ sont claires : le cas $u=0$ correspond à $a=1$, autrement dit à $\alpha=0$, le cas $v=0$ correspond à $b=1 \Leftrightarrow \beta=0$, le cas $u+v=0$ correspond à $\beta=-\alpha$, le cas $u-v=0$ à $\beta=\alpha$, le cas $2 u-v+u^{2} v=0 \Leftrightarrow v=\frac{2 u}{1-u^{2}}$ correspond à $\beta=2 \alpha$ et $2 v-u+u v^{2}=0$ à $\alpha=2 \beta$.

D'après la table 1 , les cas $\alpha=0$ et $\beta=\alpha$ se ramènent au cas $\beta=0$ par les transpositions (24) et (12) des sommets, les cas $\beta=2 \alpha$ et $\alpha=2 \beta$ se ramènent au cas $\beta=-\alpha$ par les transpositions (12) et (14) des sommets, si bien qu'il ne reste que les cas $\beta=-\alpha$ et $\beta=0$.

Dans le premier cas $M\left(S_{\alpha}, S_{0}, S_{-\alpha}\right)$, on trouve

$$
P_{M}(x)=\left(x^{4}-2(2 a+3) x^{2}+1+4 a+4 a^{2}\right)\left(x^{2}+2 a-3\right)^{2},
$$

d'où on déduit :

- $\quad$ si $-1 \leq a<-\frac{1}{2}$, alors $\mu_{0}=-\sqrt{3-2 a}$, racine double,

- $\quad$ si $a=-\frac{1}{2}$, alors $\mu_{0}=-2$, racine triple, 
- $\quad$ si $-\frac{1}{2}<a<1$, alors $\mu_{0}=-1-\sqrt{2 a+2}$, racine simple.

Dans le deuxième cas $M\left(S_{\alpha}, S_{0}, S_{0}\right)$, on trouve

$$
P_{M}(x)=\left(x^{4}-10 x^{2}-8 a+17\right)\left(x^{2}-1\right)^{2},
$$

d'où $\mu_{0}=-\sqrt{5+2 \sqrt{2 a+2}}$, qui est simple.

Concernant la dernière famille de type III, nous avons le résultat suivant.

Théorème 7.3. Si un quadruplet de $G(2,8)$ de type III a un ordre de platitude supérieur ou égal à 2 , alors, quitte à permuter ses sommets, il admet une forme réduite $M\left(R_{\alpha}, S_{0}, R_{\beta}\right)$ telle que $a:=\cos \alpha, b:=\cos \beta$ satisfont l'équation

$$
a^{2}+4 a b+b^{2}+a^{4}-4 a^{3} b-3 a^{2} b^{2}-4 a b^{3}+b^{4}+4 a^{3} b^{3}=1 .
$$

Réciproquement, pour tout $\alpha \in]-\pi, \pi]$, il existe un unique $\beta \in]-\pi, \pi]$ et un unique $c \in$ $\left[\frac{1}{3}, 1\right]$ tels que le quadruplet de $G(2,8)$ de forme réduite $M\left(R_{\alpha}, S_{0}, R_{\beta}\right)$ et de paramètre c a un ordre de platitude au moins 2 . Si $\alpha=0$ alors $\beta=\pi ;$ sinon, la valeur $b:=\cos \beta$ est la plus grande des deux solutions de (15) avec $a:=\cos \alpha$.

Preuve. Bien que le principe soit le même que pour le théorème 7.2, la preuve du théorème 7.3 est beaucoup plus longue. Le plan de cette preuve est le suivant. Une condition nécessaire pour avoir un ordre de platitude au moins 2 est qu'un discriminant s'annule. A ce stade, nous avons utilisé une première fois l'ordinateur, pour calculer et factoriser ce discriminant. On obtient les facteurs correspondant à $\alpha$ ou $\beta=0$, ainsi que deux facteurs notés $\Delta_{1}$ et $\Delta_{2}$, c.f. (20). Le facteur $\Delta_{1}$ correspond à (15). Il se trouve que le deuxième facteur $\Delta_{2}$ ne s'annule que pour $\alpha=\beta=-\frac{\pi}{2}, 0$ ou $\frac{\pi}{2}$, c.f. lemme 7.4 ci-dessous et sa preuve à la fin de l'article; nous avons eu recours une deuxième fois à l'ordinateur pour cette preuve. Le calcul explicite du spectre de la matrice de Seidel associée au quadruplet dans les cas $\alpha=0, \beta=0$ et $\alpha=\beta= \pm \frac{\pi}{2}$ montre que l'ordre de platitude du quadruplet est au plus un dans chacun de ces cas, ce qui démontre la première assertion de l'énoncé.

Nous décrivons ensuite la courbe d'équation (15). Elle est composée de deux points isolés correspondant à $\alpha=\beta=0$ ou $\pi$, et de deux branches $\mathcal{C}_{+}$et $\mathcal{C}_{-}$se coupant aux points correspondant à $\alpha=\pi-\beta=0$ ou $\pi$, c.f. lemme 7.6 et figure 2 à gauche. Nous montrons de plus que ces deux branches sont les graphes de fonctions strictement décroissantes, concave pour l'une et convexe pour l'autre.

Enfin, nous montrons dans le lemme 7.7 que seuls les points de $\mathcal{C}_{+}$correspondent à des quadruplets d'ordre de platitude au moins 2. Ainsi, pour toute valeur de $a=\cos \alpha$, une et une seule valeur de $b=\cos \beta$ peut donner lieu à un quadruplet de platitude au moins 2 , et lorsque $a \neq 1$ cette valeur est la plus grande solution de (15). On conclut avec le lemme 7.5 : parmi les deux valeurs de $\beta$ telles que $\cos \beta=b$, une et une seule conduit à un quadruplet de platitude au moins 2 .

Détaillons à présent la preuve. Soit $\left(\Gamma_{1}, \ldots, \Gamma_{4}\right)$ un tel quadruplet et soit $M:=M\left(R_{\alpha}\right.$, $\left.S_{\gamma}, R_{\beta}\right)$ une forme réduite. En conjuguant simultanément par une rotation, on se ramène 
à nouveau au cas $\gamma=0$. Nous aurons besoin par la suite des deux équivalences suivantes au sens de la définition 2.5.

$$
M\left(R_{-\alpha}, S_{0}, R_{-\beta}\right) \sim M\left(R_{\alpha}, S_{0}, R_{\beta}\right) \text { et } M\left(R_{\pi-\alpha}, S_{0}, R_{\pi-\beta}\right) \sim-M\left(R_{\alpha}, S_{0}, R_{\beta}\right) .
$$

La première est obtenue en conjuguant simultanément toutes les lignes et colonnes blocs par $S_{0}$. Concernant la deuxième, avec $P_{1}=-I$ et $P_{2}=P_{3}=P_{4}=I$ (i.e. en multipliant les premières ligne et colonne bloc par $-I)$ on obtient $-M\left(R_{\alpha}, S_{0}, R_{\beta}\right) \sim$ $M\left(-R_{\alpha},-S_{0},-R_{\beta}\right)=M\left(R_{\alpha+\pi}, S_{\pi}, R_{\beta+\pi}\right) \sim M\left(R_{\pi-\alpha}, S_{0}, R_{\pi-\beta}\right)$ en conjuguant simultanément par $S_{\pi / 2}$.

On a une troisième symétrie, obtenue après permutation des sommets $\Gamma_{2}$ et $\Gamma_{4}$ : d'après la table 1 , une forme réduite associée au quadruplet $\left(\Gamma_{1}, \Gamma_{4}, \Gamma_{3}, \Gamma_{2}\right)$ est donnée par $M\left(R_{-\beta}, S_{0}, R_{-\alpha}\right)$, et une conjugaison simultanée par $S_{0}$ aboutit à $M\left(R_{\beta}, S_{0}, R_{\alpha}\right)$.

Comme précédemment, on utilise la paramétrisation $u=\tan \frac{\alpha}{2}$ et $v=\tan \frac{\beta}{2}$, d'où

$$
M\left(R_{\alpha}, S_{0}, R_{\beta}\right)=N(u, v)=\left(\begin{array}{cccc}
0 & I & I & I \\
I & 0 & R_{\alpha} & S_{0} \\
I & { }^{t} R_{\alpha} & 0 & R_{\beta} \\
I & S_{0} & { }^{t} R_{\beta} & 0
\end{array}\right)
$$

avec

$$
\begin{gathered}
S_{0}=\left(\begin{array}{cc}
1 & 0 \\
0 & -1
\end{array}\right), \quad R_{\alpha}=\left(\begin{array}{cc}
a & -s_{a} \\
s_{a} & a
\end{array}\right), \quad R_{\beta}=\left(\begin{array}{cc}
b & -s_{b} \\
s_{b} & b
\end{array}\right), \\
\cos \alpha=a=\frac{1-u^{2}}{1+u^{2}}, \quad \sin \alpha=s_{a}=\frac{2 u}{1+u^{2}}, \\
\cos \beta=b=\frac{1-v^{2}}{1+v^{2}}, \quad \sin \beta=s_{b}=\frac{2 v}{1+v^{2}} .
\end{gathered}
$$

Les symétries (16) se traduisent par

$$
N(-u,-v) \sim N(u, v) \text { et } N\left(\frac{1}{u}, \frac{1}{v}\right) \sim-N(u, v)
$$

et la troisième symétrie montre que le quadruplet $\left(\Gamma_{1}, \Gamma_{4}, \Gamma_{3}, \Gamma_{2}\right)$ a pour matrice associée $N(v, u)$.

Comme précédemment, une condition nécessaire pour un ordre de platitude au moins 2 est que la matrice $A(u, v):=\left(1+u^{2}\right)\left(1+v^{2}\right) N(u, v)$ ait une valeur propre multiple, ce qui implique que son polynôme caractéristique $P_{u, v}$ ait un discriminant $\Delta(u, v)$ qui s'annule.

Notons au passage que $\Delta(u, v)$ est positif ou nul, quelque soit les valeurs de $u$ et $v$. En effet, la matrice $A(u, v)$ est réelle symétrique, donc a toutes ses valeurs propres dans $\mathbb{R}$, et nous avons vu dans la section 2.1 qu'un polynôme scindé sur $\mathbb{R}$ a un discriminant positif ou nul. D'après (18) et la troisième symétrie, les matrices $A(-u,-v)$ et $A(v, u)$ ont même polynôme caractéristique que $A(u, v)$, et celui de $A\left(\frac{1}{u}, \frac{1}{v}\right)$ satisfait pour tout $x \in \mathbb{R}$, $P_{u, v}(k x)=k^{8} P_{\frac{1}{u}, \frac{1}{v}}(x)$ avec $k=u^{2} v^{2}$. Ainsi, d'après (1), $\Delta$ satisfait les symétries

$$
\Delta(u, v)=\Delta(-u,-v)=\Delta(v, u)=\left(u^{2} v^{2}\right)^{56} \Delta\left(\frac{1}{u}, \frac{1}{v}\right) .
$$


Ces symétries se répercutent naturellement sur les facteurs de $\Delta$.

Une factorisation de ce discriminant donne :

$$
\Delta(u, v)=8^{16}\left(1+u^{2}\right)^{42}\left(1+v^{2}\right)^{42} u^{2} v^{2} \Delta_{1}(u, v)^{2} \Delta_{2}(u, v),
$$

avec

$$
\Delta_{1}(u, v)=u^{2}+u v+v^{2}-3 u^{3} v-4 u^{2} v^{2}-3 u v^{3}+u^{4} v^{2}+u^{3} v^{3}+u^{2} v^{4}
$$

et $\Delta_{2}$ est un polynôme de degré total 26 et de degré partiel en $u$ ou $v$ égal à 16 , donné dans la table 2 à la fin de l'article. Ce facteur ne joue aucun rôle dans notre étude, en raison du résultat suivant, dont la preuve est, elle aussi, reportée à la fin de l'article.

Lemme 7.4. Le facteur $\Delta_{2}$ (c.f.table 2 ) s'annule si et seulement si $(u, v)=(0,0),(1,1)$ ои $(-1,-1)$.

Pour chaque cas $u=v=0,1$ ou -1 , le spectre de $N(v, v)$ se calcule explicitement. On trouve :

$$
\begin{aligned}
\operatorname{Spec} N(-1,-1) & =\operatorname{Spec} N(1,1) \\
& =(-1-\sqrt{2},-\sqrt{3},-\sqrt{3}, 1-\sqrt{2},-1+\sqrt{2}, \sqrt{3}, \sqrt{3}, 1+\sqrt{2})
\end{aligned}
$$

et $\operatorname{Spec} N(0,0)=(-\sqrt{5},-1,-1,-1,-1,1, \sqrt{5}, 3)$.

Ainsi, pour ces trois valeurs de $(u, v), \mu_{0}$ est simple.

Concernant les autres facteurs de $\Delta$, on retrouve les cas $u=0$ et $v=0$ correspondant à $\alpha=0$ et $\beta=0$. Le cas $\Delta_{1}(u, v)=0$ aboutit à la condition (15) de l'énoncé. En effet, en remplaçant $a$ et $b$ par leurs expressions données par (17), on vérifie que le polynôme

$$
\varphi(a, b)=a^{2}+4 a b+b^{2}+a^{4}-4 a^{3} b-3 a^{2} b^{2}-4 a b^{3}+b^{4}+4 a^{3} b^{3}-1
$$

satisfait l'identité :

$$
\varphi(a, b)=\left(1+u^{2}\right)^{-4}\left(1+v^{2}\right)^{-4} \Delta_{1}(u, v) \Delta_{1}(-u, v) .
$$

Ceci démontre la première assertion de l'énoncé du théorème 7.3.

Lemme 7.5. Une condition nécessaire pour que le quadruplet soit d'ordre de platitude au moins 2 est que $\sin \alpha$ et $\sin \beta$ soient de même signe.

Preuve. Nous venons de voir qu'une condition nécessaire est que $\Delta_{1}(u, v)$ soit nul. En écrivant $\Delta_{1}(u, v)$ sous la forme

$$
\Delta_{1}(u, v)=\left(u^{2}+u v+v^{2}\right)(1-u v)^{2}-u v(u+v)^{2},
$$

on obtient que $\Delta_{1}(u, v)>0$ si $u v<0$. Autrement dit, le lieu où $\Delta_{1}(u, v)=0$ est entièrement dans les deux quarts de plan principaux $u v \geq 0$, c.f. figure 2 droite. On conclut avec les formules (17). 

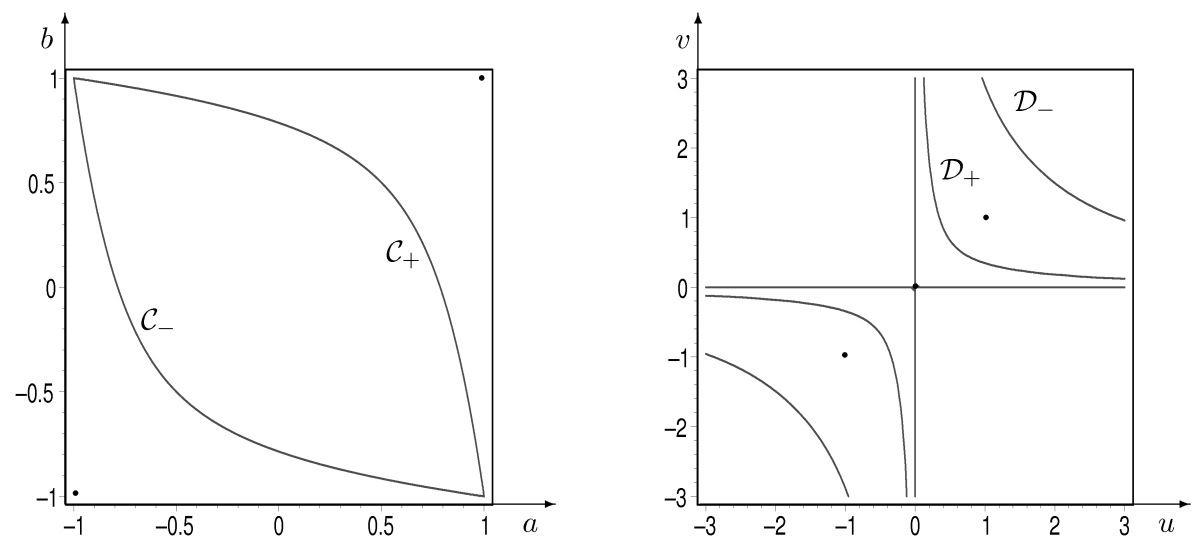

FIGURE 2. À gauche, les deux branches $\mathcal{C}_{+}$et $\mathcal{C}_{-}$et les deux points isolés $(-1,-1)$ et $(1,1)$ de la courbe algébrique $\mathcal{C}$ d'équation donnée par (15) dans le carré $[-1,1]^{2}$. Les quadruplets d'ordre de platitude 2 correspondent aux paramètres $a, b \operatorname{sur} \mathcal{C}_{+}$. A droite, le lieu où $\Delta(u, v)$ s'annule, composé des axes $u=v=0$, des points isolés $u=v=0$ ou \pm 1 annulant le facteur $\Delta_{2}$ de la table 2 et de la courbe $\mathcal{D}$ annulant le facteur $\Delta_{1}$ donné par (21), comprenant les quatre branches $\left(\mathcal{D}_{+}, \mathcal{D}_{-}\right.$et leurs symétriques) et le point isolé $(0,0)$.

Nous décrivons à présent la courbe algébrique $\mathcal{C}$ d'équation donnée par (15). La figure 2 gauche donne une allure de cette courbe dans le carré $|a|$ et $|b| \leq 1$, seul cas qui nous intéresse puisque $a$ et $b$ sont des cosinus.

Lemme 7.6. La courbe $\mathcal{C}$ d'équation (15) est composée de :

- deux points isolés $(-1,-1)$ et $(1,1)$,

- deux branches, notées $\mathcal{C}_{+}$et $\mathcal{C}_{-}$, chacune symétrique par rapport à la première bissectrice, et symétriques l'une de l'autre par rapport à l'origine, se coupant aux points $(-1,1)$ et $(1,-1)$.

La branche $\mathcal{C}_{+}$est le graphe d'une fonction (strictement) concave et décroissante

$$
\psi_{+}: \mathbb{R} \rightarrow \mathbb{R}, a \mapsto b=\psi_{+}(a)
$$

qui satisfait $\psi_{+}(1 / 2)=1 / 2$. La branche $\mathcal{C}_{-}$est le graphe de la fonction convexe et décroissante $\psi_{-}: a \mapsto-\psi_{+}(-a)$.

Preuve. Bien que nous ne soyons intéressés que par les portions de $\mathcal{C}$ dans le carré $[-1,1]^{2}$, nous décrivons $\mathcal{C}$ dans le plan $\mathbb{R}^{2}$ tout entier. D'après (16) on a les identités $\varphi(a, b)=\varphi(-a,-b)=\varphi(b, a)$, ce qui entraîne les symétries annoncées.

Par ailleurs, on a $\varphi(a, 1)=\varphi(a,-1)=\left(a^{2}-1\right)^{2}$ et de même (par symétrie) $\varphi(1, b)=$ $\varphi(-1, b)=\left(b^{2}-1\right)^{2}$. Ainsi, les seuls points de $\mathcal{C}$ sur les quatre droites $a= \pm 1$ ou $b= \pm 1$ sont les quatre points d'intersection $( \pm 1, \pm 1)$. En résumé :

$$
(|a| \text { ou }|b|=1 \text { et } \psi(a, b)=0) \Rightarrow(|a|=|b|=1) .
$$


- Au point $(1,1)$, la hessienne de $\varphi$ est

$$
H(1,1)=\left(\begin{array}{cc}
\frac{\partial^{2} \varphi}{\partial x^{2}}(1,1) & \frac{\partial^{2} \varphi}{\partial x \partial y}(1,1) \\
\frac{\partial^{2} \varphi}{\partial x \partial y}(1,1) & \frac{\partial^{2} \varphi}{\partial y^{2}}(1,1)
\end{array}\right)=4\left(\begin{array}{cc}
2 & 1 \\
1 & 2
\end{array}\right),
$$

qui est définie positive, donc $(1,1)$ est isolé. Par symétrie, il en est de même au point $(-1,-1)$.

- Au point $(-1,1)$, la hessienne de $\varphi$ est $H(-1,1)=4\left(\begin{array}{ll}2 & 7 \\ 7 & 2\end{array}\right)$, ce qui signifie que $\varphi(-1+x, 1+y)=4\left(x^{2}+7 x y+y^{2}\right)+o\left(x^{2}+y^{2}\right)=(2 y+(7-3 \sqrt{5}) x)(2 y+(7+$ $3 \sqrt{5}) x)+o\left(x^{2}+y^{2}\right)$ donc localement au voisinage de $(-1,1), \mathcal{C}$ est composée de deux branches régulières $\mathcal{C}_{+}$et $\mathcal{C}_{-}$de pentes respectivement $-\frac{7-3 \sqrt{5}}{2}$ et $-\frac{7+3 \sqrt{5}}{2}$.

Par symétrie, la situation est identique au point $(1,-1)$. Il nous reste à montrer que ces branches se recollent et forment les graphes de fonctions ayant les propriétés annoncées.

Soit $\left(a_{0}, b_{0}\right)$ un point de $\mathcal{C}$ autre que l'un des quatre points $( \pm 1, \pm 1)$. D'après (23) on a donc $\left|a_{0}\right|$ et $\left|b_{0}\right|$ différents de 1 . Nous montrons ci-dessous que $\mathcal{C}$ contient une branche régulière locale passant par $\left(a_{0}, b_{0}\right)$, qui est le graphe d'une fonction strictement monotone $b=\psi(a)$.

Pour cela, commençons par vérifier qu'aucune des dérivées partielles de $\varphi$ ne s'annule en $\left(a_{0}, b_{0}\right)$. On note $\varphi_{a}$ et $\varphi_{b}$ ces dérivées partielles. Par symétrie, il suffit de le vérifier pour $\varphi_{a}\left(a_{0}, b_{0}\right)=2\left(a_{0}+2 b_{0}+2 a_{0}^{3}-6 a_{0}^{2} b_{0}-3 a_{0} b_{0}^{2}-2 b_{0}^{3}+6 a_{0}^{2} b_{0}^{3}\right)$. Pour cela, calculons le discriminant de $\varphi$ considéré comme polynôme en $a$ à coefficients dépendant de $b$. On trouve que ce discriminant vaut

$$
-16\left(25-37 b^{2}+27 b^{4}\right)\left(4 b^{4}-2 b^{2}+1\right)^{2}(b-1)^{4}(b+1)^{4},
$$

donc ne peut s'annuler que pour $b=-1$ ou 1 . Ainsi les polynômes $\varphi\left(\cdot, b_{0}\right)$ et $\varphi_{a}\left(\cdot, b_{0}\right)$ ne peuvent pas s'annuler simultanément au même point $a_{0}$, donc $\varphi_{a}\left(a_{0}, b_{0}\right) \neq 0$. De même on a $\varphi_{b}\left(a_{0}, b_{0}\right) \neq 0$. D'après le théorème des fonctions implicites, il existe $\eta, \varepsilon>0$ (dépendant de $a_{0}$ et $b_{0}$ ) tels que pour tout $\left.a \in\right] a_{0}-\eta, a_{0}+\eta[$, il existe une unique solution $b=\psi(a)$ dans l'intervalle $] b_{0}-\varepsilon, b_{0}+\varepsilon[$ de l'équation $\varphi(a, b)=0$. De plus, quitte à diminuer $\eta$, la fonction $\psi$ est dérivable sur $] a_{0}-\eta, a_{0}+\eta\left[\right.$ et satisfait $\psi\left(a_{0}\right)=b_{0}$ et $\psi^{\prime}(a)=-\frac{\varphi_{a}}{\varphi_{b}}(a, \psi(a)) \neq 0$.

On vérifie que le point $\left(\frac{1}{2}, \frac{1}{2}\right)$ est sur $\mathcal{C}$. D'après ce qui précède, pour $a_{1}, a_{2}$ assez proches de $\frac{1}{2}, a_{1}<\frac{1}{2}<a_{2}$, il existe une fonction continue monotone $\psi_{+}$de $] a_{1}, a_{2}$ [ dans $\mathbb{R}$ telle que $\psi_{+}\left(\frac{1}{2}\right)=\frac{1}{2}$ et $\varphi\left(a, \psi_{+}(a)\right)=0$ pour tout $\left.a \in\right] a_{1}, a_{2}[$. Montrons que cette fonction se prolonge sur tout l'intervalle $[-1,1]$ et que son graphe coïncide avec la branche locale $\mathcal{C}_{+}$.

Supposons $a_{1}$ minimal avec cette propriété. Si on avait $a_{1}>-1$, alors d'après (23) on aurait $\left.\psi_{+}(a) \in\right]-1,1[$ pour tout $\left.a \in] a_{1}, \frac{1}{2}\right]$, donc par monotonie, $\psi_{+}$aurait une limite finie notée $b_{1} \in[-1,1]$ lorsque $a \rightarrow a_{1}$ et par continuité on aurait $\varphi\left(a_{1}, b_{1}\right)=0$, donc $\left.b_{1} \in\right]-1,1\left[\mathrm{~d}\right.$ 'après (23). D'après ce qui précède, le graphe de $\psi_{+}$coönciderait avec 
l'unique branche régulière de $\mathcal{C}$ passant par $\left(a_{1}, b_{1}\right)$, ce qui permettrait de prolonger $\psi_{+}$à gauche de $a_{1}$, contredisant sa minimalité. Ainsi $a_{1} \leq-1$ et $\psi_{+}$se prolonge par continuité en -1 , avec nécessairement $\psi_{+}(-1)=1$ d'après (23) et puisque les points $(-1,-1)$ et $(1,1)$ sont isolés. De manière analogue, on montre que la valeur maximale $a_{2}$ satisfait $a_{2} \geq 1$ et que $\psi_{+}$se prolonge par continuité en 1 , avec $\psi_{+}(1)=-1$. Notons que ce raisonnement n'utilise pas la particularité du point $\left(\frac{1}{2}, \frac{1}{2}\right)$.

Par symétrie, il existe de même une fonction continue monotone $\psi_{-}$de $[-1,1]$ dans $\mathbb{R}$ telle que $\psi_{-}\left(-\frac{1}{2}\right)=-\frac{1}{2}$ et $\varphi(a, \psi(a))=0$ pour tout $\left.a \in\right]-1,1[$.

Puisqu'il n'y a que deux branches locales passant par $(-1,1)$, les graphes de $\psi_{+}$et $\psi_{-}$se raccordent sur ces branches. De plus $\mathcal{C}$ ne peut pas contenir d'autre point du carré ]$-1,1\left[^{2}\right.$, puisque la branche régulière passant par ce point se prolonge au moins jusqu' aux points $(-1,1)$ et $(1,-1)$, donc coïncide soit avec $\mathcal{C}_{+}$, soit avec $\mathcal{C}_{-}$.

En dehors du carré $[-1,1]^{2}$, le même raisonnement montre que $\mathcal{C}$ est constitué uniquement des prolongements des branches $\mathcal{C}_{+}$et $\mathcal{C}_{-}$. L'examen des termes de plus haut degré de $\varphi$ montre que ces branches n'ont pas d'asymptote, mais des directions asymptotiques verticales et horizontales. Précisément on obtient

$$
\psi_{+}(a) \sim(-a / 4)^{1 / 3}, a \rightarrow-\infty, \quad \psi_{+}(a) \sim-4 a^{3}, a \rightarrow+\infty
$$

et l'inverse pour $\psi_{-}$.

Il nous reste à vérifier que les courbes $\mathcal{C}_{+}$et $\mathcal{C}_{-}$n'ont pas de point d'inflexion. En dérivant deux fois l'identité $\varphi(a, \psi(a))=0$, on obtient que les points d'inflexion d'une courbe de niveau de $\varphi$ sont donnés par l'équation

$$
J:=\varphi_{a a} \varphi_{b}^{2}-2 \varphi_{a b} \varphi_{a} \varphi_{b}+\varphi_{b b} \varphi_{a}^{2}=0
$$

et le résultant de $\varphi$ et $J$, vus comme polynômes en $a$ à coefficients dépendant de $b$, ne s'annule que pour $b=-1$ ou 1 . En effet, on a

$\operatorname{Res}(\varphi, J)=2^{12} 3^{4}\left(961-1124 b^{2}+756 b^{4}+16 b^{6}+16 b^{8}\right)\left(4 b^{4}-2 b^{2}+1\right)^{6}(b-1)^{12}(b+1)^{12}$

et le polynôme de degré $8 \mathrm{n}$ 'a pas de racine réelle, puisque $961-1124 x+756 x^{2}>0$ pour tout $x \in \mathbb{R}^{+}$. Compte tenu de la concavité de $\psi_{+}$et de ses équivalents lorsque $a \rightarrow \pm \infty$, son graphe $\mathcal{C}_{+}$ne peut pas avoir non plus d'inflexion aux points $(-1,1)$ et $(1,-1)$. Il en est de même de $\mathcal{C}_{-}$par symétrie.

Comparons à présent $\mathcal{C}$ à la courbe algébrique $\mathcal{D}$ d'équation $\Delta_{1}(u, v)=0, \Delta_{1}$ donnée par (21). La figure 2 droite donne une allure de cette courbe. A chacune des branches $\mathcal{C}_{+}$ et $\mathcal{C}_{-}$correspondent deux branches de la courbe $\mathcal{D}$. En effet, les changements $u$ en $-u$ et $v$ en $-v$ ne modifient pas les valeurs de $a$ et $b$ données par (17).

La branche supérieure $\mathcal{C}_{+}$, i.e. dans le demi-plan $a+b>0$ correspond aux deux branches "intérieures" (les plus proches de l'origine) de $\mathcal{D}$. Notons $\mathcal{D}_{+}$la branche intérieure du quadrant $u, v>0$. De même, la branche inférieure $\mathcal{C}_{-}$(dans le demi-plan $a+b<0$ ), correspondant aux deux branches "extérieures" de $\mathcal{D}$; notons $\mathcal{D}_{-}$la branche extérieure du quadrant $u, v>0$.

Par ailleurs, d'après (16) on peut supposer sans perte de généralité $0 \leq \alpha \leq \pi$. En vertu du lemme 7.5 on suppose donc désormais $\alpha$ et $\beta$ dans $[0, \pi]$. 

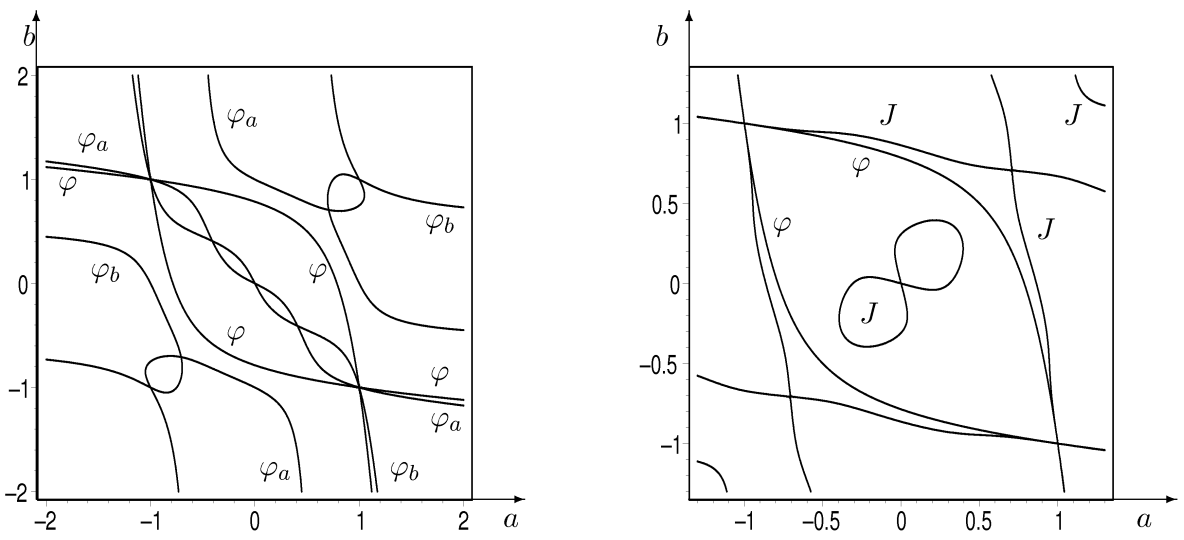

FIGURE 3. À gauche, les lieux d'annulation de $\varphi$ donné par (22) et de ses dérivées partielles $\varphi_{a}, \varphi_{b}$. A droite, le lieu d'annulation de $\varphi$ et le lieu des points d'inflexion des courbes de niveau de $\varphi$. L'allure très simple et régulière de $\mathcal{C}$ est trompeuse : les dérivées de $\varphi$ présentent des graphes plus alambiqués.

Lemme 7.7. Soit $\alpha, \beta \in[0, \pi]$ et $\mu_{0}$ la plus petite valeur propre de $M\left(R_{\alpha}, S_{0}, R_{\beta}\right)$. Notons $a=\cos \alpha$ et $b=\cos \beta$. Si le point $(a, b)$ est sur la portion $\mathcal{C}_{+}$, alors $\mu_{0}$ est valeur propre double. Si $(a, b)$ est sur la portion $\mathcal{C}_{-}$, alors $\mu_{0}$ est valeur propre simple. Si $a=-b= \pm 1$ alors $\mu_{0}=-\sqrt{5}$, qui est double.

Preuve. Nous donnons ci-dessous une preuve élémentaire et très détaillée, donc assez longue. Dans la remarque 1 qui suit cette preuve, nous esquissons une autre preuve beaucoup plus courte, mais qui utilise des outils d'analyticité et les résultats de [7].

Le principe de la preuve est le suivant : en un point explicite de $\mathcal{C}_{+}, \mu_{0}$ est bien valeur propre double. Si en un point de $\mathcal{C}_{+}$ce n'était plus le cas, alors on montre que $\mu_{0}$ serait au moins triple en un point intermédiaire, puis on montre que ceci n'est pas possible.

On se fixe dans les variables $u, v$ et on précise la notation de $\mu_{0}$ par $\mu_{0}(u, v)$; c'est la plus petite racine du polynôme caractéristique, que nous avions noté $P_{u, v}$, de la matrice $N(u, v)=M\left(R_{\alpha}, S_{0}, R_{\beta}\right)$.

Portons notre attention sur $\mathcal{D}_{+}$. L'idée est de déterminer la multiplicité de $\mu_{0}$ en un point particulier de $\mathcal{D}_{+}$et de montrer que cette multiplicité reste constante sur tout $\mathcal{D}_{+}$. Plus précisément, nous allons montrer que la multiplicité de $\mu_{0}$ est exactement 2 , et que toutes les autres valeurs propres de $N(u, v)$ sont simples. Nous abrégerons cela en disant que " $\mu_{0}$ est la seule valeur propre double". Une complication provient du fait que les zéros de $\Delta$ sont multiples, puisqu'ils sont de multiplicité paire.

Au point $p_{0}$ de $\mathcal{D}_{+}$de coordonnées $u=v=\frac{1}{\sqrt{3}}$ (correspondant au point $(a, b)=$ $\left.\left(\frac{1}{2}, \frac{1}{2}\right) \operatorname{sur} \mathcal{C}_{+}\right) P_{u, v}$ se factorise en $P=x(x-2)\left(x^{2}-2\right)\left(x^{2}-2 x-2\right)(x+2)^{2}$, ce qui donne

$$
\operatorname{Spec} M=(-2,-2,-\sqrt{2}, 1-\sqrt{3}, 0, \sqrt{2}, 2,1+\sqrt{3}) .
$$

Ainsi $\mu_{0}\left(\frac{1}{\sqrt{3}}, \frac{1}{\sqrt{3}}\right)=-2$ est la seule valeur propre double au point $p_{0} \in \mathcal{D}_{+}$. Par conti- 
nuité, il existe un petit arc $\gamma$ ouvert de $\mathcal{D}_{+}$contenant le point $p_{0}$ tel que $\mu_{0}(u, v)$ est la seule valeur propre double de $M$ pour tout $(u, v) \in \gamma$.

Supposons que $\mu_{0}$ n'est pas double en un point $q$ de $\mathcal{D}_{+}$. Alors, entre $p_{0}$ et $q$, il existe un premier point $p_{1}$ où $\mu_{0}$ cesse d'être la seule valeur propre double. Précisément, puisque $\mathcal{D}_{+}$est connexe par arcs (c'est justement un arc), il existe un chemin $c:[0,1] \rightarrow \mathcal{D}_{+}$avec $c(0)=p_{0}$ et $c(1)=q$. Soit $p_{1}=c\left(t_{0}\right)$ où

$$
t_{0}=\sup \left\{t \in[0,1] ; \forall s \in[0, t], \mu_{0}(c(s)) \text { est la seule valeur propre double }\right\} .
$$

Notons $\left(u_{1}, v_{1}\right)$ les coordonnées de $p_{1}$ et reparamétrons le chemin entre $p_{0}$ et $p_{1}$ par $\left(p_{t}\right)_{0 \leq t \leq 1}$ avec $p_{t}=\left(u_{t}, v_{t}\right)$. Puisqu'il y a changement de valeur propre double en $p_{1}$, c'est que $P_{u_{1}, v_{1}}$ a, ou bien un zéro au moins triple en $\mu_{0}\left(u_{1}, v_{1}\right)$, ou bien un deuxième zéro multiple ailleurs.

Par ailleurs, nous avons pour tout $t \in[0,1[$ :

$$
\frac{\partial P_{p_{t}}}{\partial u}\left(\mu_{0}\left(p_{t}\right)\right)=\frac{\partial P_{p_{t}}}{\partial v}\left(\mu_{0}\left(p_{t}\right)\right)=0 .
$$

En effet, si on fixe $u=u_{t}$ et que $v$ varie autour de $v_{t}$, alors le minumum local de $P_{u_{t}, v}$ proche de $\mu_{0}\left(p_{t}\right)$ doit être négatif ou nul, aussi bien lorsque $v<v_{t}$ que lorsque $v>v_{t}$, puisque $P_{u_{t}, v}$ garde toutes ses racines réelles.

Par continuité, la relation (25) reste vrai pour $t=1$. Nous démontrons à présent l'énoncé ci-dessous.

Le point $p_{1}$ est un zéro au moins triple de $\Delta$.

Pour cela, nous montrons qu'une perturbation de l'ordre de $\varepsilon$ sur $u$ et $v$ au voisinage de $u_{1}, v_{1}$ induit une perturbation de l'ordre de $\varepsilon^{3}$ au maximum sur $\Delta(u, v)$. Nous utiliserons l'énoncé suivant.

Soit $\left(P_{\varepsilon}\right)_{\varepsilon \in\left[0, \varepsilon_{0}\right]}$ une famille de polynômes scindés sur $\mathbb{R}$, tous de même degré d, telle que $P_{\varepsilon}=P_{0}+O(\varepsilon)$ sur tout compact de $\mathbb{R}$. Si $P_{0}$ a un zéro d'ordre $n$ en un point $\xi_{0} \in \mathbb{R}$, alors $P_{\varepsilon}$ a n zéros (comptés avec leurs multiplicités) en $\xi_{1, \varepsilon} \leq \cdots \leq \xi_{n, \varepsilon}$ vérifiant tous $\xi_{j, \varepsilon}=\xi_{0}+O\left(\varepsilon^{1 / n}\right)$.

En effet, on a $P_{0}(x)=\left(x-\xi_{0}\right)^{n} Q(x)$ avec $|Q| \geq a>0$ dans un voisinage complexe de $\xi_{0}$. Le théorème de dépendance continue des racines de polynômes implique que pour $\varepsilon$ assez petit, $P_{\varepsilon}$ a $n$ racines dans ce voisinage et, puisque $P_{\varepsilon}$ est scindé sur $\mathbb{R}$, ces racines sont sur l'axe réel. Pour chacune de ces racines, on a

$$
0=P_{\varepsilon}\left(\xi_{j}\right)=P_{0}\left(\xi_{j, \varepsilon}\right)+O(\varepsilon)=\left(\xi_{j, \varepsilon}-\xi_{0}\right)^{n} Q\left(\xi_{j, \varepsilon}\right)+O(\varepsilon),
$$

d'où $\xi_{j, \varepsilon}-\xi_{0}=O\left(\varepsilon^{1 / n}\right)$.

Pour alléger l'écriture, nous notons $\mu_{0}\left(p_{1}\right)$ par $\mu_{1}$.

Soit $\left(u_{\varepsilon}, v_{\varepsilon}\right)_{0<\varepsilon \leq \varepsilon_{0}}$ une famille de points de $\mathbb{R}^{2}$ (avec par exemple $\varepsilon_{0}=\frac{1}{2}$ ) telle que $u_{\varepsilon}=u_{1}+O(\varepsilon)$ et $v_{\varepsilon}=v_{1}+O(\varepsilon)$. Puisque $P_{u, v}$ est polynomial en $u$ et $v$, on a $P_{u_{\varepsilon}, v_{\varepsilon}}=P_{p_{1}}+O(\varepsilon)$ uniformément sur tout compact de $\mathbb{R}$. Au point $\mu_{1}$, puisque $p_{1}$ satisfait (25), on a une meilleure approximation : $P_{u_{\varepsilon}, v_{\varepsilon}}\left(\mu_{1}\right)=P_{p_{1}}\left(\mu_{1}\right)+O\left(\varepsilon^{2}\right)=O\left(\varepsilon^{2}\right)$. 
Distinguons les cas où $\mu_{1}$ est racine double ou de multiplicité au moins 3 de $P_{p_{1}}$.

- Si $\mu_{1}$ est un zéro double, alors on a $P_{p_{1}}^{\prime \prime}\left(\mu_{1}\right) \neq 0$. Les deux plus petites racines de $P_{u_{\varepsilon}, v_{\varepsilon}}$, notées $\xi_{1}$ et $\xi_{2}$, sont donc à une distance $O(\varepsilon)$ de $\mu_{1}$ et la plus petite racine de $P_{u_{\varepsilon}, v_{\varepsilon}}^{\prime}$, notée $\eta_{1}$, est à une distance $O\left(\varepsilon^{2}\right)$ de $\mu_{1}$. Ainsi, $\left(\xi_{1}-\eta_{1}\right)\left(\xi_{2}-\eta_{1}\right)=O\left(\varepsilon^{2}\right)$. De plus, puisque $\mu_{1}$ est double, c'est que $P_{p_{1}}$ a un autre zéro $\xi$ d'ordre de multiplicité $n \geq 2$, qui fournit $n$ zéros (éventuellement certains confondus) $\xi_{k}, \ldots, \xi_{k+n-1}$ de $P_{u_{\varepsilon}, v_{\varepsilon}}$, à une distance $O\left(\varepsilon^{1 / n}\right)$ de $\xi$ et $n-1$ zéros $\eta_{k}, \ldots, \eta_{k+n-2}$ de $P_{u_{\varepsilon}, v_{\varepsilon}}^{\prime}$, à une distance $O\left(\varepsilon^{1 /(n-1)}\right)$ de $\xi$, donc $\xi_{i}-\eta_{j}=O\left(\varepsilon^{1 / n}\right)$, donc $\prod_{\substack{k \leq i \leq k+n-1 \\ k \leq j \leq k+n-2}}\left(\xi_{i}-\eta_{j}\right)=O\left(\varepsilon^{r}\right)$ avec $r=n(n-1) \min \left(\frac{1}{n}, \frac{1}{n-1}\right) \geq 1$.

On obtient ainsi $\Delta\left(u_{\varepsilon}, v_{\varepsilon}\right)=8^{8} \prod_{\substack{1 \leq i \leq 8 \\ 1 \leq j \leq 7}}\left(\xi_{i}-\eta_{j}\right)=O\left(\varepsilon^{3}\right)$ comme annoncé.

- Si $\mu_{0}$ est d'ordre de multiplicité $n \geq 3$, alors de même $P_{u_{\varepsilon}, v_{\varepsilon}}$ a $n$ zéros (éventuellement certains confondus) $\alpha_{1}, \ldots, \alpha_{n}$ à une distance $O\left(\varepsilon^{2 / n}\right)$ de $\mu_{0}$ et $P_{u_{\varepsilon}, v_{\varepsilon}}^{\prime}$ a $n-1$ zéros $\beta_{1}, \ldots, \beta_{n-1}$, à une distance $O\left(\varepsilon^{2 /(n-1)}\right)$ de $\mu_{0}$, donc $\prod_{\substack{1 \leq i \leq n \\ 1 \leq j \leq n-1}}\left(\alpha_{i}-\beta_{j}\right)=O\left(\varepsilon^{r}\right)$ avec $r=n(n-1) \min \left(\frac{2}{n}, \frac{2}{n-1}\right)=2(n-1) \geq 4$, d'où $\Delta\left(u_{\varepsilon}, v_{\varepsilon}\right)=O\left(\varepsilon^{4}\right)$.

Puisque les autres facteurs de $\Delta$ ne s'annulent pas au voisinage de $\mathcal{D}_{+}$et que $\Delta_{1}$ est un facteur seulement double de $\Delta$, c'est que $p_{1}$ est un zéro multiple de $\Delta_{1}$, c'est-àdire un point singulier de $\mathcal{D}_{+}$. Nous obtiendrons notre contradiction après avoir démontré l'énoncé suivant.

La courbe $\mathcal{D}_{+}$est régulière.

Cela revient à montrer qu'en tout point de $\mathcal{D}_{+}$, l'une des dérivées partielles $\frac{\partial \Delta_{1}}{\partial u}$ ou $\frac{\partial \Delta_{1}}{\partial v}$ est non nulle. Il se trouve qu'aucune de ces dérivées ne s'annule. Par symétrie, il suffit de le vérifier pour $\frac{\partial \Delta_{1}}{\partial u}$, et pour le voir, le plus simple est de calculer le résultant de $\Delta_{1}$ et de $\frac{\partial \Delta_{1}}{\partial u}$ considérés comme polynômes en l'une des variables, par exemple $u$, à coefficients dépendant de $v$. On obtient

$$
\operatorname{Res}\left(\Delta_{1}, \frac{\partial \Delta_{1}}{\partial u}\right)=-v^{6}\left(15 v^{8}-8 v^{6}+386 v^{4}-8 v^{2}+15\right)\left(v^{2}+1\right)^{4},
$$

qui n'a aucune racine réelle sur $] 0,+\infty\left[\right.$ (avec $V=v^{2}+\frac{1}{v^{2}}$, le facteur de degré 8 est égal à $\left.\left(15 V^{2}-8 V+356\right) v^{4} \neq 0\right)$.

Nous avons ainsi une contradiction avec la définition de $p_{1}$, ce qui démontre la première assertion du lemme 7.7.

D'après (18), le spectre de $N(u, v)$ est l'opposé de celui de $N\left(\frac{1}{u}, \frac{1}{v}\right)$. Par conséquent, sur la branche $\mathcal{D}_{-}$c'est la plus grande valeur propre qui est double, et $\mu_{0}$ est simple, ce qui démontre la deuxième assertion.

Les spectres de $M\left(R_{0}, S_{0}, R_{\pi}\right)$ et de $M\left(R_{\pi}, S_{0}, R_{0}\right)$ sont $(-\sqrt{5},-\sqrt{5},-1,-1,1,1$, $\sqrt{5}, \sqrt{5})$, ce qui démontre la troisième assertion du lemme 7.7.

Remarques. 1. Nous donnons ci-dessous une preuve alternative du lemme 7.7, utilisant l'analyticité réelle et des résultats de [7]. Il est connu que les valeurs propres d'une matrice 
symétrique sont des fonctions analytiques des coefficients de la matrice : en quelque sorte, puisque ces valeurs propres restent réelles, il n'y a pas de ramification. Précisément, sur un arc analytique de l'espace des coefficients $\gamma:[0,1] \rightarrow \mathbb{R}^{n(n+1) / 2}$, il existe des fonctions analytiques $\lambda_{1}, \ldots, \lambda_{n}:[0,1] \rightarrow \mathbb{R}$ telles que $\operatorname{Spec}(\gamma(t))=\left\{\lambda_{1}(t), \ldots, \lambda_{n}(t)\right\}$.

Puisque les deux plus petites valeurs propres $\alpha_{1}$ et $\alpha_{2}$ sont confondues dans un voisinage du point $p_{0}$ de $\mathcal{D}_{+}$et que $\mathcal{D}_{+}$est analytique (car régulière) elles restent confondues sur tout $\mathcal{D}_{+}$. Si ces deux valeurs propres cessaient d'être les plus petites, alors une troisième valeur propre viendrait se confondre avec elles, donnant ainsi un quadruplet d'ordre de platitude au moins 3 , donc dans $\mathbb{R}^{5}$, ce qui, pour un quadruplet de type III, n'est pas possible (comme annoncé en début de partie 7).

2. Le fait qu' aucune des dérivées partielles de $\Delta_{1}$ ne s'annule sur $\mathcal{D}_{+}$entraîne que $\mathcal{D}_{+}$ est un graphe de fonction $v=f(u)$ strictement décroissante, comme le montre la figure 2 droite. On peut aussi vérifier que la fonction $f$, de graphe $\mathcal{D}_{+}$, est convexe : en notant les dérivées de $\Delta_{1}$ en indices, les points d'inflexion de la courbe $\Delta_{1}=0$ sont donnés par l'équation analogue à (24)

$$
I:=\Delta_{1, u u} \Delta_{1, v}^{2}-2 \Delta_{1, u v} \Delta_{1, u} \Delta_{1, v}+\Delta_{1, v v} \Delta_{1, u}^{2}=0
$$

et le résultant de $\Delta_{1}$ et $I$ se trouve être

$$
\begin{aligned}
& \operatorname{Res}\left(\Delta_{1}, I\right)= \\
& \quad-1125 v^{56}-2700 v^{54}-30510 v^{52}-870636 v^{50}-5119785 v^{48}-20098224 v^{46} \\
& -75922472 v^{44}-239033712 v^{42}-542135418 v^{40}-870721640 v^{38} \\
& -1011988692 v^{36}-867966696 v^{34}-553041546 v^{32}-261610992 v^{30} \\
& -95256360 v^{28}-31850288 v^{26}-11714529 v^{24}-3641964 v^{22}-636590 v^{20} \\
& -60300 v^{18}-10125 v^{16}
\end{aligned}
$$

donc n'est jamais nul sur $\mathbb{R}^{*}$.

Preuve du lemme 7.4. Pour terminer notre étude, nous devons à présent examiner le facteur $\Delta_{2}(u, v)$ donné dans la table 2. Les symétries (19) entraînent les symétries

$$
\Delta_{2}(u, v)=\Delta_{2}(v, u)=\Delta_{2}(-u,-v)=\left(u^{2} v^{2}\right)^{8} \Delta_{2}\left(\frac{1}{u}, \frac{1}{v}\right) .
$$

En particulier, c'est un polynôme symétrique en $u$ et $v$ et, pour chaque monôme, la parité en $u$ et $v$ est la même. On constate aussi que son degré total est 26 et son degré partiel en $u$ ou $v$ est égal à 16 .

Rappelons que $\Delta(u, v)$ est positif ou nul pour tout $u, v \in \mathbb{R}$. Puisque tous les autres facteurs de $\Delta$ sont des carrés, il s'ensuit que le facteur $\Delta_{2}$ est lui aussi positif ou nul.

Si l'on fixe l'une des variables, disons $v$, alors $\Delta_{2}$ peut être considéré comme un polynôme en $u$. Précisément, notons $\Delta_{2}^{v}: u \mapsto \Delta_{2}(u, v)$ ce polynôme. Puisque $\Delta_{2}^{v}(u) \geq$ 0 pour tout $u, v \in \mathbb{R}$, les zéros éventuels de $\Delta_{2}^{v}$ sont tous de multiplicité paire, donc au moins 2.

Nous allons voir que ceci implique $v=-1,0$ ou 1. 
$\Delta_{2}(u, v)=$

$$
\begin{aligned}
& \left(17280 v^{10}+69417 v^{8}+96048 v^{6}+53344 v^{4}+15104 v^{2}+6400\right) u^{16} \\
& +\left(34560 v^{11}+88056 v^{9}+14424 v^{7}-100896 v^{5}-57216 v^{3}+4608 v\right) u^{15} \\
& +\left(74880 v^{12}+316668 v^{10}+344112 v^{8}+163452 v^{6}+114896 v^{4}+63040 v^{2}+15104\right) u^{14} \\
& +\left(74240 v^{13}+188872 v^{11}-631648 v^{9}-1495680 v^{7}-938904 v^{5}-246720 v^{3}-57216 v\right) u^{13} \\
& +\left(74880 v^{14}+377910 v^{12}+148128 v^{10}+294960 v^{8}+1586256 v^{6}+1218358 v^{4}+114896 v^{2}+53344\right) u^{12} \\
& +\left(34560 v^{15}+188872 v^{13}-925168 v^{11}-2642528 v^{9}-1934112 v^{7}-1209072 v^{5}-938904 v^{3}-100896 v\right) u^{11} \\
& +\left(17280 v^{16}+316668 v^{14}+148128 v^{12}+638952 v^{10}+3210720 v^{8}+3898536 v^{6}+1586256 v^{4}+163452 v^{2}\right. \\
& +\left(88056 v^{15}-631648 v^{13}-2642528 v^{11}-3338128 v^{9}-1839312 v^{7}-1934112 v^{5}-1495680 v^{3}+14424 v\right) u^{9} \\
& +\left(69417 v^{16}+344112 v^{14}+294960 v^{12}+3210720 v^{10}+6431940 v^{8}+3210720 v^{6}+294960 v^{4}+344112 v^{2}\right. \\
& +\left(14424 v^{15}-1495680 v^{13}-1934112 v^{11}-1839312 v^{9}-3338128 v^{7}-2642528 v^{5}-631648 v^{3}+88056 v\right) u^{7} \\
& +\left(96048 v^{16}+163452 v^{14}+1586256 v^{12}+3898536 v^{10}+3210720 v^{8}+638952 v^{6}+148128 v^{4}+316668 v^{2}\right. \\
& +\left(-100896 v^{15}-938904 v^{13}-1209072 v^{11}-1934112 v^{9}-2642528 v^{7}-925168 v^{5}+188872 v^{3}+34560 v\right) u^{5} \\
& +\left(53344 v^{16}+114896 v^{14}+1218358 v^{12}+1586256 v^{10}+294960 v^{8}+148128 v^{6}+377910 v^{4}+74880 v^{2}\right) u^{4} \\
& +\left(-57216 v^{15}-246720 v^{13}-938904 v^{11}-1495680 v^{9}-631648 v^{7}+188872 v^{5}+74240 v^{3}\right) u^{3}
\end{aligned}
$$

TABLE 2. Le facteur $\Delta_{2}$ dans la factorisation de $\Delta(u, v)$ donnée dans (20).

En effet, si $\Delta_{2}^{v}$ a un zéro multiple, alors son discriminant, que nous notons $\delta(v)$, doit s'annuler. Ce discriminant est un polynôme en $v$ de degré 388 , à coefficients positifs ou négatifs, compris entre et $10^{36}$ et $6.10^{82}$ en valeur absolue, dont l'écriture développée tient en une dizaine de pages, qui se factorise en

$$
\delta(v)=2^{258} 3^{63}\left(3 v^{2}+4\right)^{3}\left(4 v^{2}+3\right)^{3}(v-1)^{16}(v+1)^{16}\left(1+v^{2}\right)^{98} v^{46} \delta_{1} \delta_{2} \delta_{3} \delta_{4}^{2} \delta_{5}^{2}
$$

avec

$$
\begin{gathered}
\delta_{1}=13 v^{2}-v+13, \quad \delta_{2}=13 v^{2}+v+13, \\
\delta_{3}=15 v^{8}-8 v^{6}+386 v^{4}-8 v^{2}+15, \quad \delta_{4}=5 v^{12}+96 v^{8}-54 v^{6}+96 v^{4}+5
\end{gathered}
$$

et $\delta_{5}$ donné dans la table 3 .

On vérifie qu'aucun des facteurs $\delta_{j}, j=1, \ldots, 5$ ne peut s'annuler. C'est immédiat pour le facteur $\delta_{5}$ puisqu'il est pair et à coefficients positifs, ainsi que pour $\delta_{1}, \delta_{2}$, de discriminant négatif; c'est clair aussi pour $\delta_{3}$ et $\delta_{4}$, en distinguant les cas $|v| \leq 1$ et $|v| \geq 1$. Il reste donc les valeurs $v=-1,0$ ou 1 . Pour terminer la preuve du lemme 7.4, il nous suffit donc de vérifier l'énoncé suivant.

Pour chaque cas $v=0,1 \mathrm{ou}-1, \Delta_{2}^{v}$ s'annule si et seulement si $u=v$.

- Pour $v=0$ c'est clair :

$$
\Delta_{2}^{0}(u)=\Delta_{2}(u, 0)=u^{6}\left(100 u^{4}+11 u^{2}+640\right)\left(4 u^{2}+3\right)^{3}
$$




$$
\begin{aligned}
& \delta_{5}=98771297640625 v^{56}+1567578397993750 v^{54}+17170447906224375 v^{52} \\
& +130609603125647750 v^{50}+915729673270688975 v^{48}+5278317278410544940 v^{46} \\
& +25129155772921615134 v^{44}+95619725848983670764 v^{42}+315165949378537365357 v^{40} \\
& +876429398364445511546 v^{38}+2007749944244141195225 v^{36}+3815953551075577709226 v^{34} \\
& +6192208079067279245075 v^{32}+8553557422601616884264 v^{30}+9638192263271293218852 v^{28} \\
& +8553557422601616884264 v^{26}+6192208079067279245075 v^{24}+3815953551075577709226 v^{22} \\
& +2007749944244141195225 v^{20}+876429398364445511546 v^{18}+315165949378537365357 v^{16} \\
& +95619725848983670764 v^{14}+25129155772921615134 v^{12}+5278317278410544940 v^{10} \\
& +915729673270688975 v^{8}+130609603125647750 v^{6}+17170447906224375 v^{4} \\
& +1567578397993750 v^{2}+98771297640625
\end{aligned}
$$

TABLE 3. Le facteur $\delta_{5}$ dans la factorisation de $\delta(v)$ donnée dans (26).

- $\quad$ Pour $v=1$, on obtient :

$$
\begin{aligned}
\Delta_{2}(u, 1)=(u-1)^{4} & \left(257593 u^{12}+1013908 u^{11}+3602226 u^{10}+6248772 u^{9}\right. \\
+ & 11048503 u^{8}+12569128 u^{7}+15454396 u^{6}+12569128 u^{5} \\
+ & \left.11048503 u^{4}+6248772 u^{3}+3602226 u^{2}+1013908 u+257593\right)
\end{aligned}
$$

On a comme prévu $\Delta_{2}(1,1)=0$. Il reste à montrer que c'est la seule valeur de $u$ qui annule $\Delta_{2}(u, 1)$, autrement dit que le facteur de degré $12, f(u):=\Delta_{2}(u, 1)(u-1)^{-4}$, est toujours strictement positif.

Pour $u \neq-1$, si on pose $U:=\frac{u-1}{u+1}$, on obtient $f(u)=\left(\frac{2}{1-U}\right)^{12} p(U)$ avec

$$
p(U)=1369 U^{12}+8336 U^{10}+23536 U^{8}+44064 U^{6}+83520 U^{4}+76032 U^{2}+20736>0 .
$$

Pour $u=-1$ on trouve $f(-1)=2^{12} 37^{2} \neq 0$.

- Pour $v=-1$, la symétrie $\Delta_{2}(-u,-v)=\Delta_{2}(u, v)$ ramène au cas $v=1$. Ceci achève la preuve du lemme 7.4.

\section{Références}

[1] L. M. Blumenthal, Theory and applications of distance geometry. Oxford Univ. Press 1953. MR0054981 (14,1009a) Zbl 0050.38502

[2] U. Brehm, The shape invariant of triangles and trigonometry in two-point homogeneous spaces. Geom. Dedicata 33 (1990), 59-76. MR1042625 (91c:53048) Zbl 0695.53038

[3] U. Brehm, B. Et-Taoui, Congruence criteria for finite subsets of complex projective and complex hyperbolic spaces. Manuscripta Math. 96 (1998), 81-95. MR1624356 (99e:51021) Zbl 0908.51001

[4] U. Brehm, B. Et-Taoui, Congruence criteria for finite subsets of quaternionic elliptic and quaternionic hyperbolic spaces. Geom. Dedicata 84 (2001), 261-269. MR1825360 (2002f:51028) Zbl 0988.51016

[5] J. H. Conway, R. H. Hardin, N. J. A. Sloane, Packing lines, planes, etc.: packings in Grassmannian spaces. Experiment. Math. 5 (1996), 139-159. MR1418961 (98a:52029) Zbl 0864.51012 
[6] B. Et-Taoui, Equiangular lines in $\mathbb{C}^{r}$. Indag. Math. (N.S.) 11 (2000), 201-207. MR1813161 (2002a:51022) Zbl 0983.51010

[7] B. Et-Taoui, Equi-isoclinic planes of Euclidean spaces. Indag. Math. (N.S.) 17 (2006), 205219. MR2321381 Zbl 1103.51011

[8] B. Et-Taoui, Equi-isoclinic planes in Euclidean even dimensional spaces. Adv. Geom. 7 (2007), 379-384. MR2339265 (2008f:51025) Zbl 1133.51009

[9] A. Fruchard, Les triplets de la variété de Grassmann $G_{2}\left(\mathbb{R}^{6}\right)$. Geom. Dedicata 68 (1997), 123-144. MR1484560 (99e:51023) Zbl 0889.51026

[10] J. Haantjes, Equilateral point-sets in elliptic two- and three-dimensional spaces. Nieuw Arch. Wiskunde (2) 22 (1948), 355-362. MR0023530 (9,369c) Zbl 0037.21703

[11] S. G. Hoggar, New sets of equi-isoclinic n-planes from old. Proc. Edinburgh Math. Soc. (2) 20 (1976/77), 287-291. MR0467505 (57 \#7361) Zbl 0371.50004

[12] P. W. H. Lemmens, J. J. Seidel, Equi-isoclinic subspaces of Euclidean spaces. Indag. Math. 35 (1973), 98-107. MR0313926 (47 \#2478) Zbl 0272.50008

[13] P. W. H. Lemmens, J. J. Seidel, Equiangular lines. J. Algebra 24 (1973), 494-512. MR0307969 (46 \#7084) Zbl 0255.50005

[14] J. J. Seidel, Discrete non-Euclidean geometry. In: Handbook of incidence geometry, 843-920, North-Holland 1995. MR1360730 (96m:52001) Zbl 0826.51012

[15] J. R. Sylvester, Determinants of block matrices. 2005. http://amath.colorado.edu/courses/7400/2005fall/006/sylvester.pdf

[16] J. H. van Lint, J. J. Seidel, Equilateral point sets in elliptic geometry. Indag. Math. 28 (1966), 335-348. MR0200799 (34 \#685) Zbl 0138.41702

[17] Y.-C. Wong, Isoclinic $n$-planes in Euclidean $2 n$-space, Clifford parallels in elliptic $(2 n-1)$ space, and the Hurwitz matrix equations. Mem. Amer. Math. Soc. No. 41 (1961), iii+112. MR0145437 (26 \#2968) Zbl 0124.13401

Received 22 January, 2007

B. Et-Taoui, A. Fruchard, Laboratoire de Mathématiques, Informatique et Applications, Faculté des Sciences et Techniques, Université de Haute Alsace, 4 rue des Frères Lumière, 68093 Mulhouse cedex, France

Email: Boumediene.Ettaoui@uha.fr, Augustin.Fruchard@uha.fr 